\title{
Reactor-Specific Spent Fuel Discharge Projections: 1984 to 2020
}
C. M. Heeb
R. A. Libby
G. M. Holter

April 1985

Prepared for the U.S. Department of Energy under Contract DE-AC06-76RLO 1830

Pacific Northwest Laboratory Operated for the U.S. Department of Energy by Battelle Memorial Institute 


\title{
DISCLAIMER
}

This report was prepared as an account of work sponsored by an agency of the United States Government. Neither the United States Government nor any agency thereof, nor any of their employees, makes any warranty, express or implied, or assumes any legal liability or responsibility for the accuracy, completeness, or usefulness of any information, apparatus, product, or process disclosed, or represents that its use would not infringe privately owned rights. Reference herein to any specific commercial product, process, or service by trade name, trademark, manufacturer, or otherwise, does not necessarily constitute or imply its endorsement, recommendation, or favoring by the United States Government or any agency thereof. The views and opinions of authors expressed herein do not necessarily state or reflect those of the United States Government or any agency thereof.

\author{
PACIFIC NORTHWEST LABORATORY \\ operated by \\ BATTELLE \\ for the \\ UNITED STATES DEPARTMENT OF ENERGY \\ under Contract DE-AC06-76RLO 1830
}

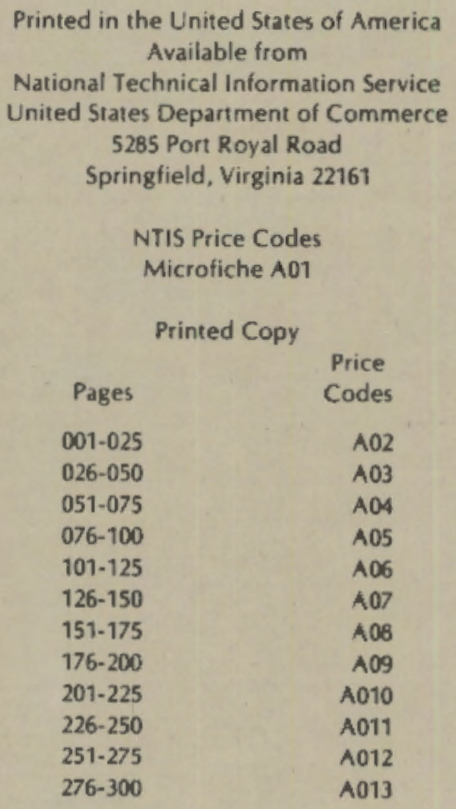


REACTOR-SPECIFIC SPENT FUEL DISCHARGE

PROJECTIONS: 1984 TO 2020

C. M. Heeb

R. A. Libby

G. M. Holter

Apri 1985

Prepared for

The Office of Civilian Radioactive Waste Management, U.S. Department of Energy

under Contract DE-AC06-76RLO 1830

Pacific Northwest Laboratory

Richland, Washington 99352 


\section{ACKHOWLEDGHEHTS}

The authors thank B. M. Cole, K. A. Ekblaw, E. T. Merrill, W. L. Purcell, R. G. Rau and 月. K. White for their assistance and valuable advice in the performance and documentation of the work described herein. Thanks are also due to S. A. Bullock for the physical preparation of the report. 


\section{CONTENTS}

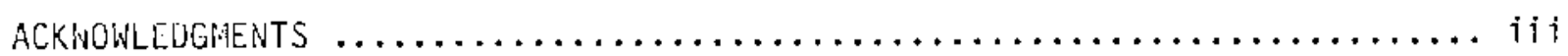

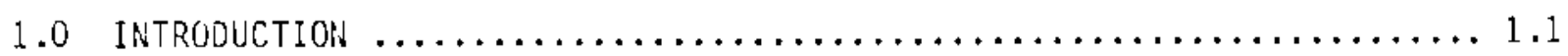

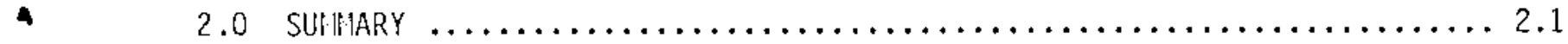

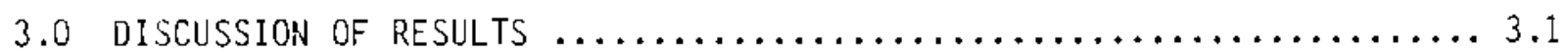

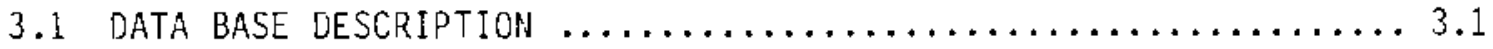

3.2 DATA base AdJUSTHENT PROCEDURE $\ldots \ldots \ldots \ldots \ldots \ldots \ldots \ldots \ldots \ldots \ldots \ldots \ldots \ldots \ldots$

3.2.1 Step 1 - Shift Utility Data Base Start-up

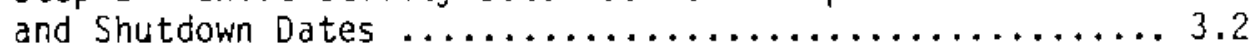

3.2.2 Step 2 - Calculation of Electrical Energy Generation from the SFDB $\ldots \ldots \ldots \ldots \ldots \ldots \ldots \ldots \ldots \ldots \ldots \ldots \ldots$

3.2.3 Step 3 - Determination of EIA and SFDB Annual

Energy Adjustment Ratio ........................ 3

3.2.4 Step 4 - Adjustment of Utility Discharges

to ilatch EIA Energy Projections $\ldots \ldots \ldots \ldots \ldots \ldots \ldots \ldots .5$

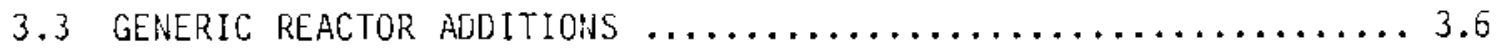

3.4 ADJUSTED DATA BASE ACCURACY $\ldots \ldots \ldots \ldots \ldots \ldots \ldots \ldots \ldots \ldots \ldots \ldots \ldots \ldots$

3.5 DATA BASE ADJUSTINENT RESULTS $\ldots \ldots \ldots \ldots \ldots \ldots \ldots \ldots \ldots \ldots \ldots \ldots \ldots \ldots \ldots \ldots \ldots$

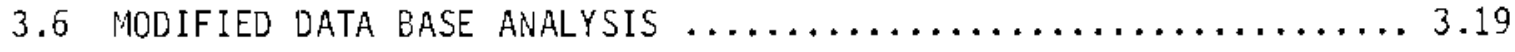

3.6.1 Spent Fuel Discharge Projections $\ldots \ldots \ldots \ldots \ldots \ldots \ldots \ldots .19$

3.6.2 Spent Fuei Storage Requirements $\ldots \ldots \ldots \ldots \ldots \ldots \ldots \ldots .21$

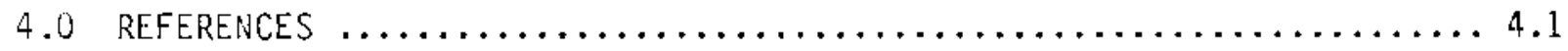

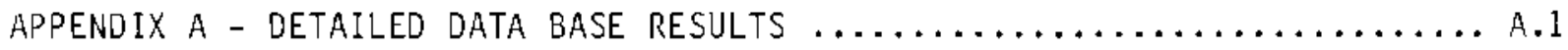

APPENDIX B - MAP OF FEDERAL ENERGY REGIONS $\ldots \ldots \ldots \ldots \ldots \ldots \ldots \ldots \ldots \ldots \ldots \ldots \ldots$ 


\section{FIGURES}

1 Spent Fuel Data Base Adjustment Process $\ldots \ldots \ldots \ldots \ldots \ldots \ldots \ldots \ldots \ldots .2$

2 Installed EIA Nuclear Capacity Projections $\ldots \ldots \ldots \ldots \ldots \ldots \ldots \ldots \ldots$

3 Projected EIA Nuclear Energy Generation $\ldots \ldots \ldots \ldots \ldots \ldots \ldots \ldots \ldots \ldots .14$

4 Projected Cumulative Spent Fuel Inventory: Adjusted SFDB

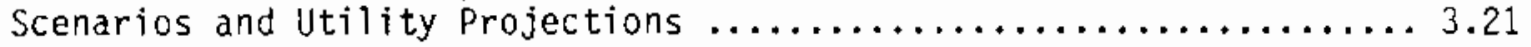

5 Projected Additional Cumulative Storage Requirements Maximum At-Reactor Storage Capacity: Adjusted Data Bases

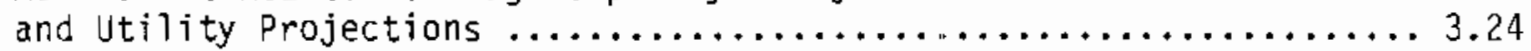

6 Projected Additional Cumulative Storage Requirements Maximum At-Reactor Storage Capacity PIus Transshipment: Adjusted Data Bases and Utility Projections ......................26 


\section{TABLES}

1 Cycle to Batch Energy Ratio by Cycle $\ldots \ldots \ldots \ldots \ldots \ldots \ldots \ldots \ldots \ldots$

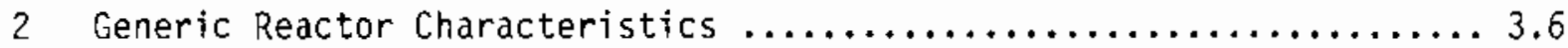

3 Regional Sites for Generic Reactors $\ldots \ldots \ldots \ldots \ldots \ldots \ldots \ldots \ldots \ldots \ldots \ldots$

41995 Projected Energy Generation by Federal Region ...............8

5 No New Orders Case Capacity and Energy Comparison $\ldots \ldots \ldots \ldots \ldots \ldots . .12$

6 No New Orders Case Adjustments to Base Exposure ................. 3.15

7 Middle Case Capacity and Energy Comparison $\ldots \ldots \ldots \ldots \ldots \ldots \ldots \ldots \ldots$

8 Middle Case Adjustments to Base Exposure $\ldots \ldots \ldots \ldots \ldots \ldots \ldots \ldots \ldots .17$

9 Utility to EIA Required Energy Adjustment Factors--EIA/SFDB ...... 3.18

10 Cumulative Spent Fuel Discharges (MTIHM) $1984-2020 \ldots \ldots \ldots \ldots \ldots .20$

11 Cumulative Additional Storage Requirements -

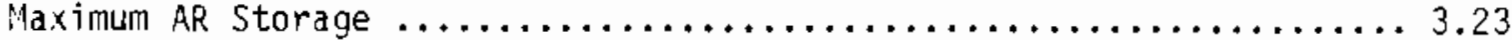

12 Cumulative Additional Storage Requirements (MTIHM) Maximum AR Storage Capacity Płus Transshipment ................ 3.25 
f

i 


\subsection{INTRODUCTION}

Under the provisions of the Nuclear Waste Policy Act of 1982 (NWPA), the Department of Energy (DOE) is responsible for the management and ultimate permanent disposal of the civilian radioactive waste generated as a result of comercial nuclear power plant operations in the U.S. The office of Civilian Radioactive Waste Managenent (OCRWM) has been established within DOE to plan for and carry out this responsibility.

The greatest portion of the radioactive waste covered under this government responsibility will be spent nuclear fuel discharged from commercial nuclear power plants. Because most of the spent fuel that will ultimately require disposal has not yet been generated, planning for the management and disposal of this spent fuel must be based primarily on projections of future spent fuel discharges fron these plants.

The DOE Energy Information Administration (EIA) annually publishes projections of nuclear energy generation on an overall, industry composite basis. (1) These EIA energy projections provide several scenarios representing different assumptions about the future growth of nuclear energy capacity in the U.S. lultiple scenarios allow analysis of the sensitivity of results and decisions to varying assumptions.

The EIA nuclear energy projections provide the basis for planning by OCRWM. However, the projections do not contain the specific reactor-by-reactor information that is needed to perform detailed anaiyses of, for example, waste system logistics, requirements for interim storage of spent fuel, or anticipated variations in the physical characteristics of the spent fuel to be received for disposal. Therefore, to provide a more detailed basis for such analyses, this study was performed to develop detailed reactor-specific spent fuel discharge projections corresponding to the EIA nuclear energy growth projections.

The basic source of data used to develop the reactor-specific information was the Spent Fuel Data Base (SFDB) maintained by Pacific Northwest Laboratory (PNL) for the DOE Commercial Spent Fuel Management (CSFM) Progran. This data base, which is updated annually, is based on information supplied directly by 
the nuclear utilities. The SFDB consists of detailed historical and projected data on reactor operation, spent fuel discharges and shipments, and spent fuel storage and handling capabilities.

The CSFM Program uses this SFDB for a number of planning purposes, including the preparation of annual projections of future requirements for additional spent fuel storage capacity. (2) The data base also provides the basic information on spent fuel for inclusion in the DOE Integrated Data Base (IDB) maintained by Oak Ridge National Laboratory. (3)

Two of the EIA nuclear growth scenarios were chosen as bases for developing the reactor-specific spent fuel discharge projections: the lliddle Case and the No New Orders Case. The Middle Case has been previously selected by the OCRIM as the base case for waste management planning purposes. The No New orders case was included to provide an additional data set which could be used to analyze the sensitivity of analytical results and management decisions to possible growth reductions in the United States nuclear industry.

The No New Orders Case includes only nuclear power plants that are currently operating and a subset of the plants currently under construction. EIA assumes that some of the plants currently under construction will not be completed, and that others will be delayed beyond official utility startup estimates. The Middle Case includes additional generic power plants not currentiy on order but scheduled to start operation in 2001. These plants will provide sufficient power generation capacity to correspond to the overali nuclear energy production rates in the EIA projections. In addition, for plants now under construction, startup dates are earlier and the assumed number of canceled piants is lower than for the No New Orders Case.

The detailed information in the CSFM Program's SFDB could not be used directly to provide reactor-specific information corresponding to the $E I A$ nuclear growth projections. This is because EIA and the individual utilities make different assumptions about the future online availabilities (i.e., capacity factors) of the individual power plants and the projected startup dates for plants under construction. Therefore, adjustments in the data were required to properly match the reactor capacity factors and startup dates to the EIA assumptions. 
For the lifdde Case, a further requirement was the specification of generic power plants to be added to the existing reactor population starting in 2001 to meet the overali installed capacity assumptions implicit in the EIA projections. Reactor types consistent with currently existing power plants were chosen to represent these generic power plants. This permitted detailed information for the generic power plants to be obtained from the SFDB in the same manner as for the currently existing or planned reactors. 


\subsection{SUMMARY}

The original spent fuel utility data base (SFDB) has been adjusted to produce agreement with the EIA nuclear energy generation forecast for the Middle Case. (1) An adjusted data base conforming to the EIA No New Orders Case was also produced to provide for less optimistic forecasts of future nucjear capacity growth than the EIA Middle Case.

The procedure developed allows the detail of the utility data base to remain intact, while the overall nuclear generation is changed to match any uniform nuclear generation forecast. This procedure adjusts the weight of the reactor discharges as reported on the SFDB and makes a minima? (less than 10\%) change in the original discharge exposures in order to preserve discharges of an integral number of fuel assemblies.

The procedure used in developing the reactor-specific spent fuel discharge projections, as well as the resulting data bases themselves, are described in detail in this report. Discussions of the procedure cover the following topics:

- a description of the data base;

- data base adjustment procedures;

- addition of generic power reactors; and

- accuracy of the data base adjustments.

Adjustment of the utility data base to match the No New Orders Case and the Middle Case has reduced the spent fuel discharge and storage requirements projections compared to the original SFDB. This reduction is due to the lower estimates of plant capacity factors and to the more delayed estimates of plant availability dates in the EIA forecasts relative to the original utility data.

Reactor-specific discharge and storage requirements for the Middle Case are presented in Appendix A. Annual and cumulative discharge projections are provided. Annual and cumulative requirements for additional storage are shown for the maximum at-reactor (AR) storage assumption, and for the maximum AR with 
transshiprent assumption. These compare directly :o the storage requirements from the utility-supplied data, as reported in the Spent Fuel storage Requirements Report. (2)

The reactor-specific spent fuel discharge projections contained in this report cover the period from 1984 through 2020. This covers a total cumulative spent fuel inventory of nearly 125,000 MTIHM (metr'c tons initial heavy metal) for the Middle Case and just over 90,000 MTIHM for the No New Orders Case. However, the methodology used can also be extended to provide consistent projections covering longer periods of time (e.g., projections up to a total of 140,000 MTIHM of spent fuel would cover the nominal capacity of the first two civilian nuclear waste repositories), or to provide detailed projections corresponding with other EIA growth scenarios (i.e., the High Case or the Low case).

The results presented in this report for the Middle Case include:

- the disaggregated spent fuel discharge projections; and

- disaggregated projections of requirements for additional spent fuel storage capacity prior to 1998.

Descriptions of the methodology and the results are included in the next section of this report. Details supporting the discussions in the main body of the report, including descriptions of the capacity and fuel discharge projections, are included in the Appendix $A$. 


\subsection{DISCUSSIDH OF RESULTS}

The SFDB is a compendium of information on U.S. commercial power reactor spent fuel and other reactor-specific information. It is based on data provided by the utility owners and represents their estimate of the amounts and characteristics of the spent fuel discharges. Electrical energy generation may be derived from the spent fuel quantities and spent fuel exposure contained in the data base.

\subsection{DATA BASE DESCRIPTION}

The SFDB contains a file for each reactor. The first portion of the file consists of time-independent information such as the location, various power ratings, dates of start-up and final shutdown, and detailed information on fuel stored in the reactor's pool(s). The second portion consists of the historica? record of fuel discharges by batch or sub-batch with discharge exposure range, number of assemblies, and the uranium mass for each batch. This, and succeeding portions, are organized by operating cycle (i.e., by the period between successive discharges). The third portion of the SFDB contains projected discharge information for 1984 and beyond.

The electrical energy generation implicit in the spent fuel data base is entirely independent of the nuclear energy generation forecasts made by EIA. This report describes how the SFDB was adjusted to be consistent with these nuclear energy generation forecasts. The technical steps involved in adjusting the data base to conform to the EIA projections are described in this section. The principal requirement of the adjustment was to retain as much of the detailed utility estimates of exposure, plant capacity factor, and discharge schedules as possible, while matching the EIA electrical energy generation forecast.

\subsection{DATA BASE ADJUSTMENT PROCEDURE}

The steps taken in the adjustment procedure are shown in Figure 1. Processing steps are shown in rectangular blocks which are assigned a number in the upper left hand corner of the block. Data bases are shown as slanted 


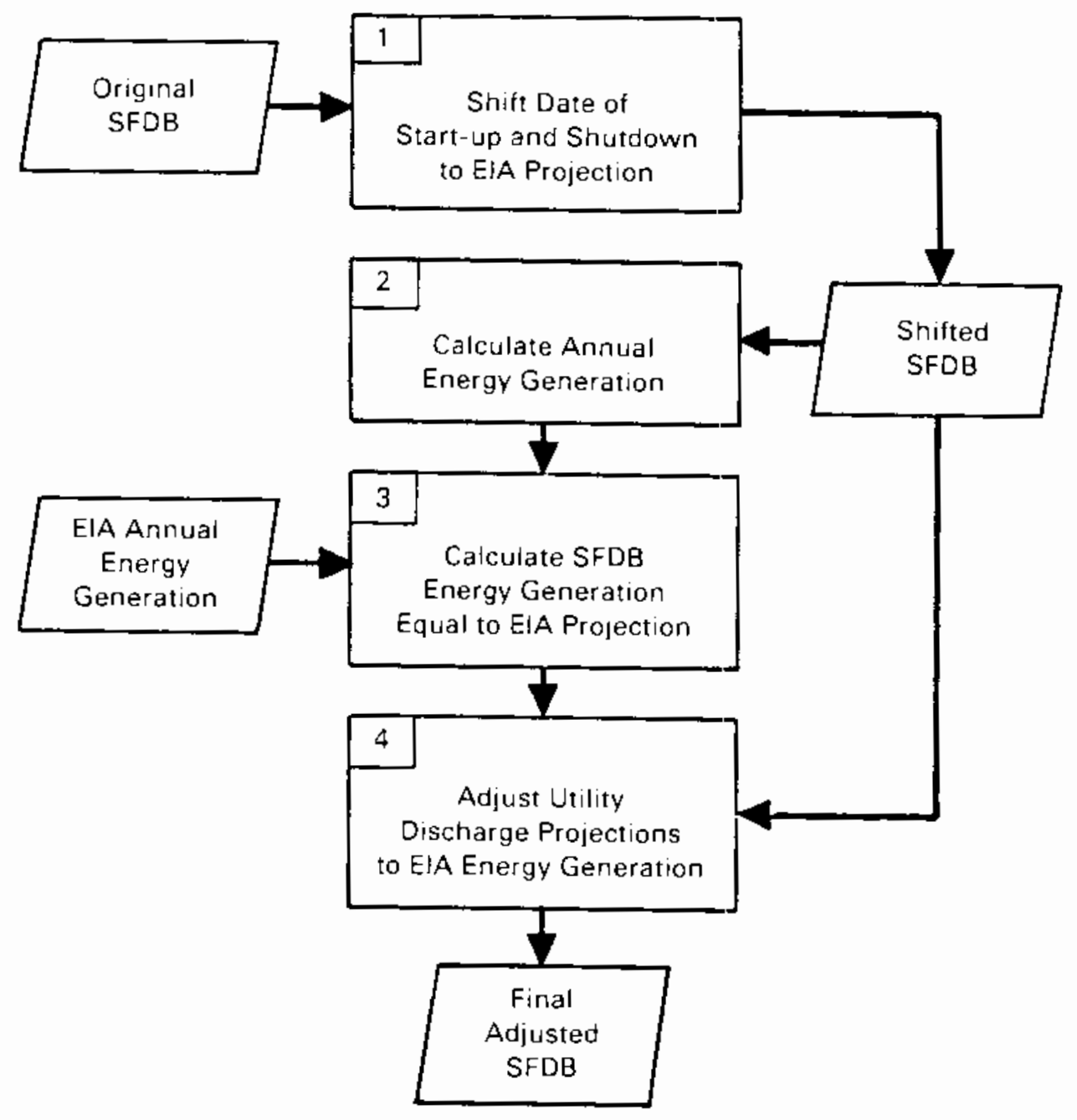

FIGURE 1. Spent Fuel Data Base Adjustment Process

blocks. The adjustment process required the construction of four major processors. Each of these processors is described in the sections that follow. The adjustment process is concerned entirely with the time beyond 1983, since historical information is left unchanged by the adjustment process.

\subsubsection{Step 1 - Shift Utility Data Base Start-up and Shutdown Dates}

Reactors starting up from 1984 to 2020 according to the EIA schedule were checked against the utility-supplied start-up dates on the SFDB. If the dates were different, the start-up date on the SFDB was shifted to a date six months prior to the EIA date of first commercial power generation. Thus the new date used was the date of first electrical generation, which by EIA convention is 
six months prior to the date of first commercial power generation. The start-up date for each reactor is shown in Appendix A, Table A.1.

The SFDB discharge dates prior to the EIA start-up date were, of course, eliminated. However the discharged batches from the eliminated dates were moved to the nearest SFDB date after the new start-up date. In cases where that date was "too close" to the start-up date, that date was eliminated and the next discharge date was chosen to receive the discharge batch collection. The criteria used to judge whether the first adjusted discharge date was "too close" was the utility estimate of the plant capacity factor. If the capacity factor jased on the adjusted discharge date greater than 0.90 , the next available utility-supplied date was selected. Operation from start-up to the new first discharge date was assumed to be at the utility-supplied capacity factor. In cases where the cycle length was longer than the utility-supplied cycle, the energy generation estimated by this procedure is greater than the utilityprojected energy generation. Additional fuel is added to the original discharge estimate to reflect this added energy generation. This procedure leaves the majority of the utility discharge dates unchanged and preserves the utility estimate of the first cycle capacity factor.

The shutdown dates were adjusted to agree with the EIA data. In accordance with EIA ground rules, the full core was assumed to be discharged in the year following the last energy generation. Next the generic reactors were added, if required and as described in Section 3.3 to match installed nuclear generation capacity. The installed capacity match was accomplished at the end of Step 1.

\subsubsection{Step 2 - Calculation of Electrical Energy Generation from the SFDB}

The cycle-energy generation from the SFDB was calculated by adding the product of discharge exposure and mass of the discharged batch for each batch discharged from the cycle. For reactors at equilibrium, the energy generated by the discharged fuel during its residence in the core is equal to the energy generated by the entire core during the cycle of discharge. To illustrate for a three batch core fuel management plan at equilibrium, each in-core fuel batch generates a fixed fraction of core power during equilibrium operation: F1, F2, F3. If $E$ is the energy generation during each equilibrium cycle, the energy 
generated by the batch scheduled for discharge, ED, would be the sum of the energy generated by the discharge batch during each of the three cycles of residence:

$$
E D=E \times F 1+E \times F 2+E \times F 3 .
$$

By factoring out $E$, the equation is expressed as follows:

$$
E D=E \times(F 1+F 2+F 3) .
$$

Since the sum of $F 1+F 2+F 3$ is 1.0 by definition, the discharge energy equals the energy generated during each equilibriur cycle:

$$
E D=E .
$$

This would be true for any core equilibrium replacement fraction. For reactors undergoing initial startup, the ratio of first cycle energy generation to first-discharge batch-energy generation will not be unity, but will approximate the reciprocal of the core fraction discharged if power sharing by in-core batches is proportional to batch size. Thus for one third core replacement, the ratio of first-cycle energy to the energy generated by the fuel in the first discharge would approximate 3 . The second discharge ratio would approximate half of this, or 1.5. The third discharge ratio would approximate 1 , and would remain 1 for all subsequent discharges.

In actual practice power sharing between in-ccre fuel batches is not exactly equitable, and fuel management plans do not specify that the same fraction of the core will be replaced for every refueling outage. However, when large numbers of reactors are involved, and when over-all energy generation over several years during which only a small fraction of the total energy generation is from plants not at equilibrium is of primary interest, average non-equilibrium ratios of cycle energy to batch energy may be approximated with sufficient accuracy. Detailed fuel management plans for several reactors were analyzed to obtain a more realistic ratio of cycle to batch energy. The ratios 
of cycle energy to batch energy were calculated and the results are summarized in the following table of factors:

TABLE 1. Cycle to Batch Energy Ratio by Cycle

First Second Third Fourth Equilibrium

PWR

2.857

1.266

1.095

1.073

1.000

BWR

3.663

1.404

1.111

1.058

1.000

The energy of a given cycle is calculated by adding the product of exposure and mass discharged over all batches from the cycle and then multiplying that result by the appropriate factor from the preceding table.

\subsubsection{Step 3 -Determination of EIA and SFDB Annual Energy Adjustment Ratio}

The shifted SFDB energy is calculated for each plant in each cycle. The cycle energies are then allocated to each year according to the number of months in the year for that cycle. The annual energy generation projected by EIA is then compared to the annual sum of energy generation from the shifted SFDB. An annual correction factor equal to the EIA energy generation divided by the SFDB energy is then calculated. The target annual energies are then used in the final adjustment process.

\subsubsection{Step 4 - Adjustment of Utility Discharges to Match EIA Energy Projections}

The projected SFDB discharge amounts are modified to be consistent with the target energies. The adjustment assumptions are that no discharge batches of less than eleven assemblies are adjusted, and that a slight adjustment to the utility-supplied exposure is made to preserve an integral number of discharge assemblies in the batches of eleven or more assemblies. Adjusting only batches greater than ten assemblies assures that no exposure will be adjusted by more than ten percent in order to preserve an integral number of assemblies. The larger batch exposures are adjusted a little more than originally called for in order to compensate for the unadjusted fuel batches having ten or less assembl-es. The original adjustment ratio determined in step 3 is preserved by this process. 


\subsection{GENERIC REACTOR ADDITIONS}

The SFDB contains information only on those reactors which were operating, or were in the planning stage by some U.S. utility in 1984. In order to meet EIA energy generation forecast after 1984 for the Middle Case, it is necessary to include generic reactors in the shifted data base at stage one in Figure 1. Two actual reactors were selected to represent the generic PWR and BWR. Both were nominal 1100 Mwe plants and both were on an 18 month refueling schedule. The PWR design exposure was $35455 \mathrm{MWD} / \mathrm{MTIHM}$, and the BWR burnup was $30400 \mathrm{MWD} /$ MTIHM. Relevant details for the two generic plant types are shown in Tabie 2.

Increases in capacity do not in general represent the addition of an integral number of plants, each with a fixed plant capacity. Fractional additions to capacity were represented by delayed start-up of one of the plants of each plant type which were otherwise started up in January each year, to maintain the correct cumulative capacity. Thus if the accumulated new capacity was equivalent to 15.65 generic plants by a given year, one of the plants would be started up in the fourth month of that year: $(1.0-0.65) \times 12.0=4.2$. The start-up of integral additions to capacity was in accordance with the EIA convention of commercial operation additions in July of each year, with a six month interval between first electrical generation and commercial operation.

It is important for logistics modeling to maintain reasonable geographic accuracy for the projected spent fuel discharges. This requires that the generic reactors be added with regional diversity. Assumption of a single site in each geographic region which would contain all generic reactors allocated to

TABLE 2. Generic Reactor Characteristics

\begin{tabular}{lcc} 
& PWR & BWR \\
\cline { 2 - 3 } Rated Power Level & $1100 \mathrm{MWe}$ & $1100 \mathrm{WW}$ \\
Thermal Efficiency & $33.0 \%$ & $33.0 \%$ \\
Fuel Cycle Length & 18 Months & $18 \mathrm{Months}$ \\
Equilibrium Enrichinent & $3.5 \mathrm{wt} \%$ & $3.0 \mathrm{Wt} \%$ \\
Discharge Exposure & $35,455 \mathrm{MWD} / \mathrm{MTIHM}$ & $30,400 \mathrm{MWD} / \mathrm{MTIHM}$ \\
Discharge Months & April, October & February, August
\end{tabular}


the region provides the necessary diversity for logistics models if regional sites near the geographic centroid of existing reactors are chosen. For this study, one such site was selected for each federal region. A map of the federat regions is shown in Appendix B. The site selected in each region is shown in Table 3 by existing reactor name. It is not expected that these sites will actually ever contain all or even any of the generic reactors located in the rejion. The sites were selected only because they are close to the center of existing reactors in each region.

The number of reactors added to each region was determined by apportioning the plants according to the projected electricity generation in 1995 from nuclear power plants in the region. It is assumed that a similar proportion of the nuclear generated electricity will be generated in the future as was generated in 1995. Table 4 shows the projected electricity generation in 1995 for each region and each region's percent of the total generation. Appendix Table A.2 shows the actual federal region placement and date of commercial operation for each generic reactor.

TABLE 3. Regional Sites for Generic Reactors

\begin{tabular}{rll} 
Federal Region & & \multicolumn{1}{c}{ Generic Reactor Site } \\
\cline { 2 - 3 } II & New England - Millstone \\
III & New York/New Jersey - Indian Point \\
IV & South Atlantic - Bellefonte \\
$V$ & Midwest - Cook \\
VI & Southwest - Commanche Peak \\
VII & Central - Cooper \\
VIII & North Central -No Site (<1/Reactor) \\
IX & West - San Onofre \\
$X$ & Northwest - WNP -2
\end{tabular}


TABLE 4. 1995 Projected Energy Generation by Federal Region

\begin{tabular}{|c|c|c|}
\hline Federal Region & $\begin{array}{c}\text { Energy } \\
\text { Generation (TWh) }\end{array}$ & $\begin{array}{l}\text { Percent } \\
\text { of Total }\end{array}$ \\
\hline I & 42.6 & 6.6 \\
\hline I I & 52.2 & 8.1 \\
\hline I I I & 74.9 & 11.6 \\
\hline IV & 187.5 & 29.2 \\
\hline V & 134.7 & 20.9 \\
\hline VI & 47.0 & 7.3 \\
\hline VII & 22.5 & 3.5 \\
\hline VIII & 0.9 & 0.1 \\
\hline IX & 52.3 & 8.1 \\
\hline$x$ & 28.2 & 4.4 \\
\hline
\end{tabular}

\subsection{ADJUSTED DATA BASE ACCURACY}

The adjustment procedure is not a numerically exact process. Approximations were required during the assignment of annual energies to operating cycle energies. A linear weighting scheme was used to assign the annual energy to an operating cycle in proportion to the number of months of that year that are in the cycle.

Using the following array and index definitions, the adjustment procedure approximation can be expressed in general terms:

$\mathrm{I}=$ cycle index, $\mathrm{J}=$ year index, $\mathrm{R}=$ reactor index

$M(I, J, R)=$ months in cycle $I$, year $J$, reactor $R$

$E D(I, R)=$ shifted data base energy for cycle $I$, reactor $R$

$E A(J)=$ annual target energy generation, year $\mathrm{J}$.

The annual shifted data base energy for reactor $P$ is calculated using the equation:

$$
J E D(J, R)=\sum_{I} M(I, J, R) / 12 \times E D(I, R) .
$$


The annual energies are then summed over reactors:

$$
\operatorname{SED}(J)=\sum_{R} J E D(J, R)
$$

A ratio of annual target energies is calculated:

$$
\operatorname{RATIO}(\mathrm{J})=\operatorname{EA}(\mathrm{J}) / \operatorname{SED}(\mathrm{J})
$$

An effective ratio is calculated for each reactor cycle using $C L(I, R)$, the cycle length in months for cycle $I$, reactor $R$ :

$$
\text { ERATIOI I, R })=\sum_{J}[(M(I, J, R) / C L(I, R)) \times \operatorname{RATIO}(J)]
$$

The effective ratio is used to produce an adjusted data base energy for cycle $I$, reactor $R$ and the discharged amounts are changed by the same ratio:

$$
\operatorname{ADJED}(I, R)=\operatorname{ERATIO}(I, R) \times \operatorname{ED}(I, R) .
$$

This process produces an adjusted data base. To test the accuracy of the adjusted data base, the adjusted cycle energies were reallocated to years, added up over all the reactors operating in a given year and compared with the target energy, $E A(J)$, in test year, $J$. In other words, the following hypothetical equality was tested for each year, $\mathrm{J}$ :

$$
\begin{gathered}
\sum \operatorname{YADJED}(J, R) \stackrel{?}{=} \operatorname{EA}(J) \text {, where } \\
R \\
\operatorname{YADJED}(J, R)=\sum_{I} M(I, J, R) / 12 \times \operatorname{ADJED}(I, R) .
\end{gathered}
$$

To determine the mathematical conditions under which this is a valid equation YADJED $(J, R)$ can be analyzed by substituting the array definitions into the preceding equation which defines $\operatorname{YADJED}(J, R)$ : 


$$
\begin{aligned}
& \sum_{R} \operatorname{YADJED}(J, R)= \\
& \frac{\sum_{\mathrm{R}} \sum_{\mathrm{I}} M(I, J, R) / 12 \times E D(I, R) \times \sum_{\mathrm{J}} M(I, J, R: / C L(I, R) \times E A(J)}{\sum_{\mathrm{R}} \sum_{I} M(I, J) / 12 \times E D(I, R)}
\end{aligned}
$$

The double summation over $R$ and $I$ in the numerator is identical to the double sum in the denominator. When the expression $\sum_{j} M(I, J, R) / C L(I, R) \times E A(J)$ reduces to $E A(u)$, the adjustment is exact.

The array $C L(I, R)$ is the cycle Jength in months. If $M(I, J, R)$ is summed over $J$ years for a given reactor cycle, it must equal the cycle length:

$$
C L(I, R)=\sum_{j} M(I, J, R)
$$

The sum can be reduced to unity for each value of I and $R$ only if all of the annual target energies in the sum EA(J) are equal and can be factored out of the sum. When this assumption is made the fraction reduces to EA(J) and the adjustment method is exact. Thus, the adjusted SFDB annual energy will equal the target EIA energy exactly only if those energies are equal for a period of time before and after the test year. Forecasts wherein the annual energy generation changes in abrupt steps will be less accurately adjusted. It is therefore a mandatory step in the adjustment process to calculate the accuracy of the adjustment for each adjusted data base.

The data base adjustment process allows a complete check on the accuracy of the approximation. When the final adjusted data base is produced, the annual adjusted energies can be calculated and summed over all reactors by performing Step 2. The resulting annual sum can be compared to the target annual energies by performing Step 3 . The resulting annual energy adjustment ratios are generally very close to unity. Thus, the adjustment process converges to within a few percent after a single iteration.

The adjustment process approximation can be explained by a numerical example. If an eighteen month cycle were made up of three months of the first year, tweive months of the next year and three months of the third year, the cycle energy would be: 


$$
\text { ECYCLE }=3 / 12 \times E 1+12 / 12 \times E 2+3 / 12 \times E 3,
$$

where E1, E2, and E3 are the annual energies of the first, second and third years respectively. If the annual energies are the target EIA energies, then ECYCLE would be the cycle energy generation on the data base that would exactly match the target energies. Now if ESFDB is the unadjusted cycle energy, the adjustment ratio would be ECYCLE/ESFDB. After the adjustment is made, ESFDB is replaced by ECYCLE, and al1 discharges are adjusted by the adjustment factor ECYCLE/ESFDB. The al location of cycle energy to years would be EADJ1 $=3 / 18 \mathrm{x}$ ECYCLE for the first year. The original allocation is $3 / 12 \times E 1$. If the adjustment were exact, then the ratio of adjusted first year allocation, EADJ1, to $3 / 12 \times E l$ should equal unity. The ratio is:

$$
\frac{3 / 18 \times(3 / 12 \times E 1+12 / 12 \times E 2+3 / 12 \times E 3)}{3 / 12 \times E 1}
$$

This expression will be unity only if El $=E 2=E 3$.

\subsection{DATA BASE ADJUSTMENT RESULTS}

The results of adjustments to the SFDB are highlighted in this section. Specifically the comparison between the target EIA capacities and energy generation, and the after-adjustment capacities and energy generation is shown. It is impossible to achieve numerical identity for two basic reasons. First, the data sources are different. The utilities supply the plant capacity numbers in the SFDB. EIA sources are independent, and while there may be differences in some detail, the overall agreement should be quite close. Second, the energy generation adjustment algorithm is not exact where adjacent year target energies differ greatly. This generally produces some minor differences between the target energy and the energy used to adjust spent fuel amounts.

Table 5 shows the installed capacity and energy generated by the target EIA forecast and the SFDB No New orders Case. The agreement is within two percent for both energy and capacity. Figure 2 shows a plot of the installed capacity and Figure 3 shows a plot of the nuclear energy generation projections. 
TABLE 5. No New Orders Case Capacity and Energy Comparison

\begin{tabular}{|c|c|c|c|c|}
\hline \multirow[b]{2}{*}{ Year } & \multicolumn{2}{|c|}{$\begin{array}{c}\text { Installed Capacity } \\
\text { (Gigawatts) }\end{array}$} & \multirow{2}{*}{$\begin{array}{l}\text { Electrical } \\
\text { (Billion }\end{array}$} & \multirow{2}{*}{$\begin{array}{l}\text { Energy } \\
\text { k!h) } \\
\text { SFOB } \\
\end{array}$} \\
\hline & EIA & SFDB & & \\
\hline 1984 & 68.0 & 67.2 & 3013.0 & 308.5 \\
\hline 1985 & 80.4 & 79.6 & $3 E 1.6$ & 350.5 \\
\hline 1986 & 87.0 & 86.1 & 392.8 & 396.7 \\
\hline 1987 & 96.7 & 95.7 & 453.3 & 457.1 \\
\hline 1988 & 103.4 & 102.5 & $5 C 5.3$ & 503.9 \\
\hline 1989 & 104.7 & 103.7 & 536.0 & 536.5 \\
\hline 1990 & 105.8 & 104.8 & 553.2 & 552.4 \\
\hline 1991 & 107.0 & 106.4 & 564.2 & 561.6 \\
\hline 1992 & 107.0 & 107.0 & 568.1 & 570.7 \\
\hline 1993 & 108.2 & 108.1 & 583.9 & 581.3 \\
\hline 1994 & 108.2 & 108.2 & 586.3 & 588.7 \\
\hline 1995 & 108.2 & 108.2 & 596.5 & 593.9 \\
\hline 1996 & 108.2 & 108.2 & 597.3 & 599.5 \\
\hline 1997 & 108.2 & 108.2 & 606.8 & 606.6 \\
\hline 1998 & 108.2 & 108.2 & 616.3 & 613.9 \\
\hline 1999 & 108.2 & 108.2 & 616.3 & 616.5 \\
\hline 2000 & 108.2 & 108.2 & 616.3 & 616.2 \\
\hline 2001 & 108.2 & 108.2 & 616.3 & 616.2 \\
\hline 2002 & 108.2 & 108.0 & 616.3 & 616.0 \\
\hline 2003 & 108.1 & 108.0 & 615.3 & 615.2 \\
\hline 2004 & 108.1 & 108.0 & 615.3 & 614.6 \\
\hline 2005 & 108.1 & 108.0 & 615.3 & 615.5 \\
\hline 2006 & 108.0 & 108.0 & 614.9 & 615.0 \\
\hline 2007 & 108.0 & 108.0 & 617.9 & 614.7 \\
\hline 2008 & 108.0 & 107.6 & 614.9 & 614.0 \\
\hline 2009 & 107.6 & 106.6 & 612.7 & 613.3 \\
\hline 2010 & 105.9 & 105.3 & 603.2 & 603.4 \\
\hline 2011 & 105.3 & 104.4 & 593.7 & 599.9 \\
\hline 2012 & 99.4 & 98.6 & 565.3 & 567.0 \\
\hline 2013 & 90.9 & 90.2 & 517.6 & 518.6 \\
\hline 2014 & 73.6 & 73.1 & 419.2 & 417.8 \\
\hline 2015 & 68.0 & 67.6 & 387.3 & 386.3 \\
\hline 2016 & 60.2 & 59.8 & $34 ? .7$ & 341.9 \\
\hline 2017 & 56.3 & 56.1 & 320.7 & 321.0 \\
\hline 2018 & 53.0 & 52.8 & 301.6 & 301.8 \\
\hline 2019 & 48.6 & 48.5 & 276.9 & 277.0 \\
\hline 2020 & 48.6 & 48.5 & 276.9 & 276.9 \\
\hline
\end{tabular}




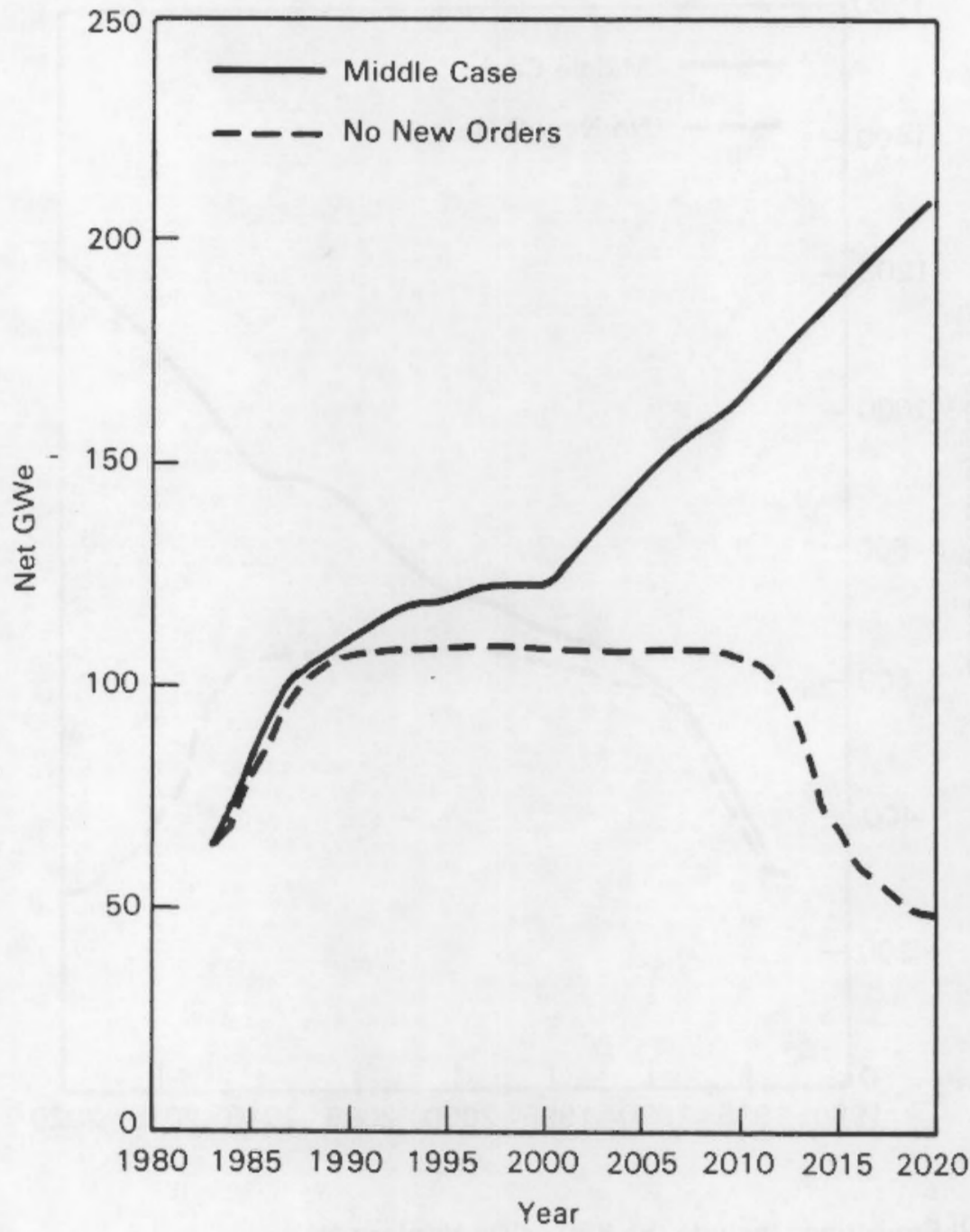

All Projections Include the 850-MWe Hanford-N Reactor

FIGURE 2. Installed EIA Nuclear Capacity Projections 


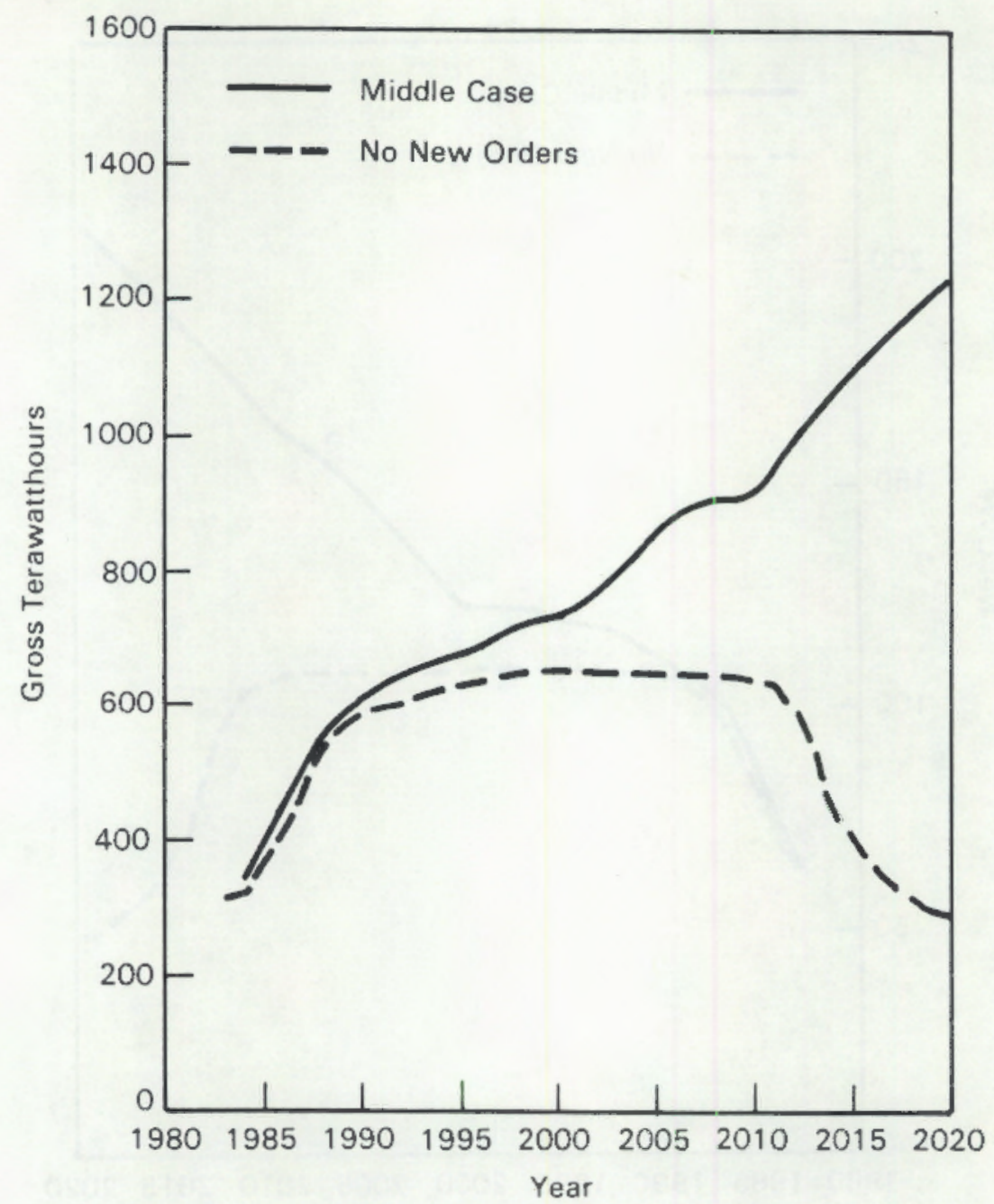

All Projections Include the 850-MWe Hanford- $\mathrm{N}$ Reactor, Which is Assumed to Generate 3.5 TWh Annually Until its Retirement

FIGURE 3. Projected EIA Nuclear Energy Generation 
Table 6 shows the extent to which the utility exposures had to be changed to produce an integral number of assemblies for the No New Orders Case. Over $77 \%$ of the adjustments were $1 \%$ or less. All adjustments were less than $10 \%$.

Table 7 shows annual Middle Case capacity and energy generation values. These are also plotted in Figures 2 and 3 . The capacities agree to within 1.3 percent. The energy generations are also generally within 1 percent of the EIA target except for the period around the year 2010. Here the decrease in annual target energy in 2009, followed by steep increases in capacity additions afterwards results in a numerical approximation of 3.3 percent which is the largest error produced in any application of the adjustment procedure. Pursuit of better agreement is possible by applying the adjustment process repeatedly. However, in view of the uncertainty inherent in forecast events around 2010, a decision was made to not pursue better agreement.

TABLE 6. No New Orders Case Adjustments to Base Exposure

\begin{tabular}{|c|c|}
\hline Adjustment Range (\%) & Assemblies \\
\hline $0.0-1.0$ & 238990 \\
\hline $1.0-2.0$ & 54689 \\
\hline $2.0-3.0$ & 8324 \\
\hline $3.0-4.0$ & 2401 \\
\hline $4.0-5.0$ & 820 \\
\hline $5.0-6.0$ & 753 \\
\hline $6.0-7.0$ & 793 \\
\hline $7.0-8.0$ & 796 \\
\hline $8.0-9.0$ & 302 \\
\hline $9.0-10.0$ & 70 \\
\hline
\end{tabular}


TABLE 7. Middle Case Capacity and Energy Comparison

Installed Capacity

(Gigawatts)

Year

1984

1985

1986

1987

1988

1989

1990

1991

1992

1993

1994

1995

1996

1997

1998

1999

2000

2001

2002

2003

2004

2005

2006

2007

2008

2009

2010

2011

2012

2013

2014

2015

2016

2017

2018

2019

2020

\begin{tabular}{|c|c|}
\hline EIA & SFDB \\
\hline 72.7 & 72.8 \\
\hline 83.9 & 84.0 \\
\hline 93.4 & 93.5 \\
\hline 103.4 & 103.4 \\
\hline 103.9 & 104.0 \\
\hline 106.3 & 106.3 \\
\hline 110.0 & 110.0 \\
\hline 112.3 & 112.3 \\
\hline 116.0 & 116.0 \\
\hline 118.3 & 118.2 \\
\hline 118.3 & 118.2 \\
\hline 118.3 & 118.2 \\
\hline 120.8 & 120.4 \\
\hline 122.1 & 121.6 \\
\hline 121.9 & 121.4 \\
\hline 121.9 & 121.4 \\
\hline 121.8 & 121.3 \\
\hline 127.1 & 127.9 \\
\hline 132.3 & 133.4 \\
\hline 137.6 & 137.9 \\
\hline 142.8 & 142.3 \\
\hline 148.1 & 147.2 \\
\hline 151.6 & 151.0 \\
\hline 155.2 & 154.6 \\
\hline 158.7 & 157.2 \\
\hline 162.3 & 162.0 \\
\hline 165.8 & 165.2 \\
\hline 170.5 & 168.3 \\
\hline 175.1 & 173.3 \\
\hline 179.8 & 177.8 \\
\hline 184.4 & 183.3 \\
\hline 189.1 & 187.7 \\
\hline 193.6 & 193.3 \\
\hline 198.2 & 197.7 \\
\hline 202.7 & 201.0 \\
\hline 207.3 & 205.4 \\
\hline 211.8 & 210.9 \\
\hline
\end{tabular}

Electrical Energy (Billion kWh)

\begin{tabular}{rrr}
\cline { 1 - 1 } EIA & & SFDB \\
\cline { 1 - 1 } 318.3 & & 321.4 \\
376.5 & & 378.5 \\
422.9 & & 427.5 \\
385.3 & & 488.2 \\
527.3 & & 526.6 \\
555.7 & & 553.0 \\
577.9 & & 576.7 \\
588.1 & & 589.6 \\
610.6 & & 614.2 \\
633.6 & & 630.4 \\
639.8 & & 642.6 \\
654.4 & & 652.6 \\
660.0 & & 660.7 \\
679.2 & & 679.2 \\
692.7 & & 691.3 \\
695.9 & & 695.9 \\
698.0 & & 694.6 \\
714.9 & & 716.6 \\
735.9 & & 741.4 \\
761.7 & & 768.3 \\
787.3 & & 787.9 \\
814.3 & & 820.8 \\
835.4 & & 843.3 \\
849.6 & & 854.4 \\
860.6 & & 869.7 \\
855.3 & & 879.6 \\
877.0 & & 906.8 \\
906.6 & & 924.1 \\
944.9 & & 955.7 \\
975.4 & & 988.7 \\
1003.3 & & 1010.9 \\
1036.0 & & 1044.4 \\
1068.3 & & 1072.6 \\
1094.1 & & 1096.6 \\
1119.9 & & 1121.1 \\
1145.2 & & 1146.1 \\
1170.0 & & 1172.0 \\
& &
\end{tabular}


Table 8 shows the Middle Case exposure adjustment results. Over 75 percent of the adjustments were 1 percent or less to produce an integral number of assemblies.

Table 9 shows the annual EIA to SFDB ratio that was used to adjust the discharge amounts for the No New Orders Case and the Middle Case. In general, the plant capacity factors assumed by EIA were lower than those from the utilities. The ratios are therefore less than unity by 10 to 15 percent.

TABLE 8. Middle Case Adjustments to Base Exposure

$\begin{array}{cc}\text { Adjustment Range (\%) } & \begin{array}{r}\text { Number of } \\ \text { Assemblies }\end{array} \\ 0.0-1.0 & 940922 \\ 1.0-2.0 & 245083 \\ 2.0-3.0 & 12612 \\ 3.0-4.0 & 3424 \\ 4.0-5.0 & 2034 \\ 5.0-6.0 & 44220 \\ 6.0-7.0 & 4864 \\ 7.0-8.0 & 297 \\ 8.0-9.0 & 135 \\ 9.0-10.0 & 50\end{array}$


TABLE 9. Utility to EIA Required Energy Adjustment Factors--EIA/SFDB

\begin{tabular}{|c|c|c|}
\hline Year & $\begin{array}{c}\text { No New Orders } \\
\text { Case }(\%) \\
\end{array}$ & Middle Case $(\%)$ \\
\hline 1984 & 86.0 & 88.7 \\
\hline 1985 & 86.6 & 85.5 \\
\hline 1986 & 83.6 & 83.0 \\
\hline 1987 & 84.2 & 83.2 \\
\hline 1988 & 83.3 & 85.7 \\
\hline 1989 & 87.3 & 87.0 \\
\hline 1990 & 87.0 & 87.2 \\
\hline 1991 & 85.7 & 86.4 \\
\hline 1992 & 86.3 & 86.6 \\
\hline 1993 & 88.4 & 88.1 \\
\hline 1994 & 88.2 & 87.5 \\
\hline 1995 & 89.8 & 89.3 \\
\hline 1996 & 89.8 & 89.6 \\
\hline 1997 & 81.4 & 90.9 \\
\hline 1998 & 92.8 & 92.1 \\
\hline 1999 & 92.3 & 92.0 \\
\hline 2000 & 92.5 & 92.6 \\
\hline 2001 & 92.7 & 91.1 \\
\hline 2002 & 92.5 & 90.3 \\
\hline 2003 & 91.8 & 89.4 \\
\hline 2004 & 92.3 & 89.9 \\
\hline 2005 & 92.6 & 89.3 \\
\hline 2006 & 92.4 & 89.0 \\
\hline 2007 & 92.5 & 88.6 \\
\hline 2008 & 93.2 & 88.0 \\
\hline 2009 & 93.5 & 86.3 \\
\hline 2010 & 93.0 & 86.5 \\
\hline 2011 & 92.1 & 86.6 \\
\hline 2012 & 91.9 & 87.1 \\
\hline 2013 & 94.2 & 86.6 \\
\hline 2014 & 95.7 & 86.9 \\
\hline 2015 & 94.5 & 87.4 \\
\hline 2016 & 96.7 & 87.6 \\
\hline 2017 & 95.5 & 87.8 \\
\hline 2018 & 95.4 & 88.0 \\
\hline 2019 & 94.6 & 88.1 \\
\hline 2020 & 93.4 & 88.3 \\
\hline
\end{tabular}




\subsection{MODIFIED DATA BASE ANALYSIS}

The final modified SFDB can be used to analyze any scenario previously analyzed using the utility data base. Results of two SFDB analyses will be presented in the final portion of this document. The first analysis will cover projected cumulative spent fuel discharges, while the second analysis will cover projected additional spent fuel storage requirements.

\subsubsection{Spent Fuel Discharge Projections}

Utility projections of cumulative spent fuel discharges through 1993 were reported in the Spent Fuel Storage Requirements Report. (2) Extending this analysis using the projections from both the unmodified utility data and the modified data base results in the data presented in the following tables and figures. Table 10 shows the cumulative spent fuel discharge projections for the utility data base and the adjusted No New Orders and Middle Case data bases from 1984 through 2020. This data is plotted in Figure 4. It is evident that the utility projections are always higher than the No New Orders Case. This is due to the delay in plant start-ups in the No New Orders projections and to the lower operating plant capacity factor assumptions. The Middle Case projections are higher than the No New Orders Case for two reasons: first, it is assumed that the start-up delays in the Middle Case are not as great as for the No New Orders Case; and second, after the year 2000 additional generic reactor are assumed to begin operation. The discharge projections are roughly equal to utility projections in the year 2010 and then begin to significantly exceed them in following years.

Annual reactor-by-reactor discharge projections for the Middle Case are shown in Table A.3. Table A.4 shows the cumulative inventory at each reactor for the Middle Case. 
TABLE 10. Cumulative Spent Fuel Discharges (MTIHM) $1984-2020$

\begin{tabular}{|c|c|c|c|}
\hline Year & Utility Data & $\begin{array}{c}\text { No New } \\
\text { Orders Case } \\
\end{array}$ & Middle Case \\
\hline 1983 & 10142 & 10142 & 10142 \\
\hline 1984 & 11425 & 11301 & 11307 \\
\hline 1985 & 13185 & 12701 & 12769 \\
\hline 1986 & 15210 & 14127 & 14297 \\
\hline 1987 & 17483 & 15716 & 16027 \\
\hline 1988 & 20209 & 17684 & 18089 \\
\hline 1989 & 22677 & 19641 & 20204 \\
\hline 1990 & 25403 & 21758 & 22354 \\
\hline 1991 & 28189 & 23957 & 24602 \\
\hline 1992 & 30915 & 26125 & 26923 \\
\hline 1993 & 33853 & 28396 & 29317 \\
\hline 1994 & 36761 & 30687 & 31945 \\
\hline 1995 & 39554 & 32984 & 34443 \\
\hline 1996 & 42517 & 35390 & 37087 \\
\hline 1997 & 45467 & 37798 & 39804 \\
\hline 1998 & 48219 & 40152 & 42403 \\
\hline 1999 & 51149 & 42557 & 45113 \\
\hline 2000 & 54074 & 45015 & 47916 \\
\hline 2001 & 56663 & 47233 & 50463 \\
\hline 2002 & 59684 & 49816 & 53463 \\
\hline 2003 & 62629 & 52307 & 56438 \\
\hline 2004 & 65545 & 54713 & 59478 \\
\hline 2005 & 68452 & 57187 & 62917 \\
\hline 2006 & 71576 & 59585 & 66145 \\
\hline 2007 & 74697 & 61947 & 69995 \\
\hline 2008 & 78275 & 64346 & 74004 \\
\hline 2009 & 80935 & 66817 & 78852 \\
\hline 2010 & 83578 & 69299 & 83059 \\
\hline 2011 & 85846 & 71783 & 87325 \\
\hline 2012 & 88189 & 74899 & 91430 \\
\hline 2013 & 90274 & 77665 & 95223 \\
\hline 2014 & 92625 & 81144 & 99560 \\
\hline 2015 & 94651 & 83175 & 103456 \\
\hline 2016 & 96647 & 85223 & 107419 \\
\hline 2017 & 98428 & 86782 & 111690 \\
\hline 2018 & 100013 & 88344 & 115928 \\
\hline 2019 & 101557 & 89801 & 120190 \\
\hline 2020 & 103140 & 90756 & 124672 \\
\hline
\end{tabular}




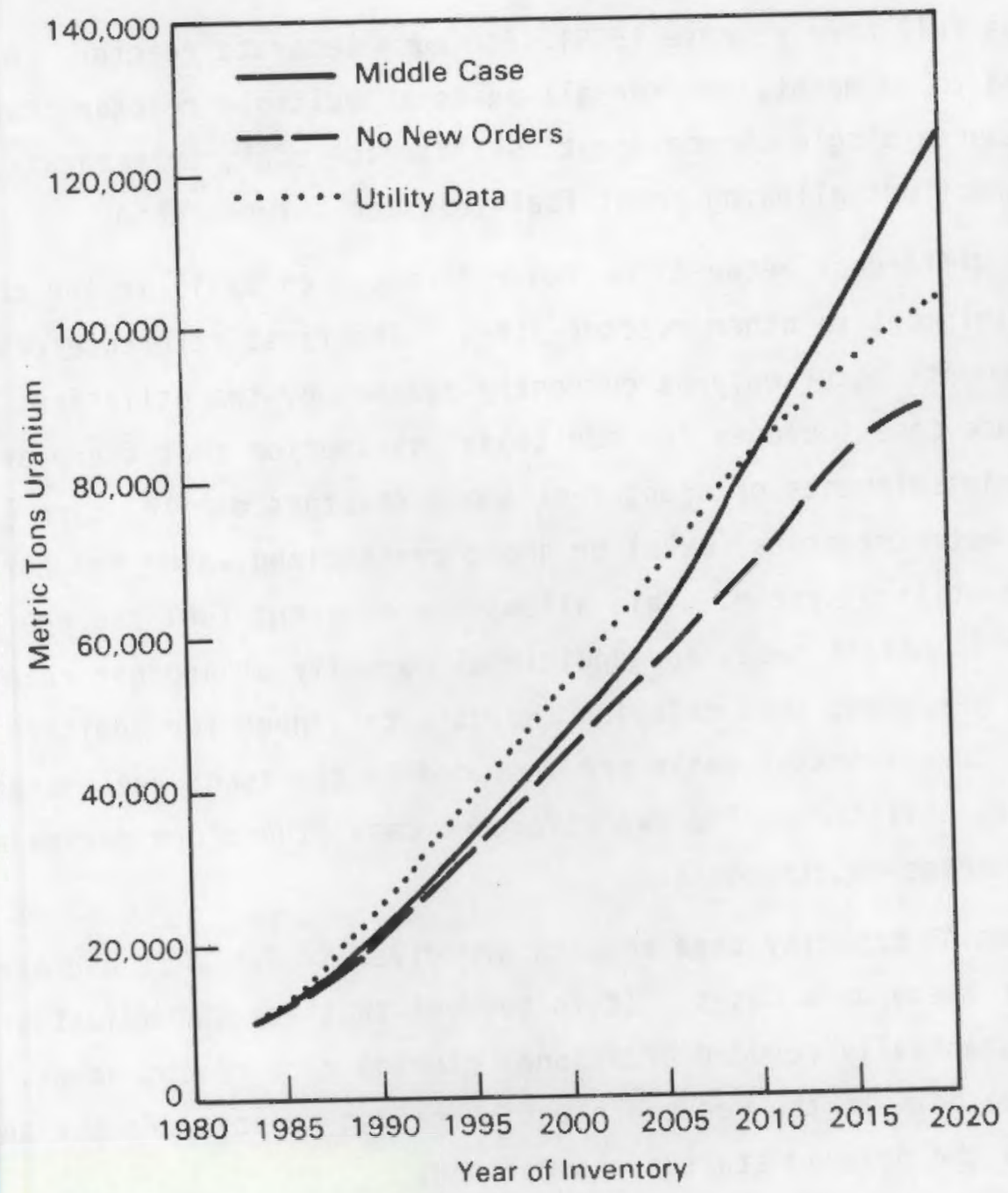

FIGURE 4. Projected Cumulative Spent Fuel Inventory: Adjusted SFDB Scenarios and Utility Projections

\subsubsection{Spent Fuel Storage Requirements}

The final result to be reported is the analysis of additional fuel storage requirements. In the Spent Fuel Storage Requirements Report several cases were analyzed using utility data over the 1984 through 1993 time period. (2) This analysis will extend the two reference cases from that report over the 1984 through 1997 period for all three data bases.

The two reference cases are based on the maximum AR storage capacities of the individual reactor pools, as determined by the utilities. Both cases include allowances for maintaining full core discharge capability, also 
referred to as full core reserve (FCR), for each separate reactor. A single FCR is assumed to be maintained for all units at multiple reactor stations employing either a single common spent fuel storage pool, or separate pools with interconnections allowing spent fuel transfer between them.

The only difference between the two references cases is in the consideration of transshipment to other reactor sites. The first reference case assumes that transshipments occur only as currently planned by the utilities. The second reference case includes the additional assumption that there are no constraints on transshipments of spent fuel among reactors of like type [i.e., among boiling water reactors (BWRs) or among pressurized water reactors (PWRs)] within a given utility system. This allows unused spent fuel storage capacity at one reactor to offset needs for additional capacity at another reactor in the same utility system, thus delaying the utility's need for additional storage capacity. Such transshipments are included in the spent fuel management plans of several utilities. The two reference cases therefore define a range of potential storage requirements.

The maximum AR capacity case results are given in Table 11 and are plotted in Figure 5 for these data bases. It is evident that the two adjusted data bases have substantially reduced additional storage requirement needs. This reduction is due both to the reduced plant operating capacity factor and (in later years) to the delayed startup assumptions.

The maximum AR capacity with intra-utility transshipment case results are given in Table 12 and are plotted in Figure 6. Again, a substantially reduced additional storage requirement need is evident under the adjusted data base energy generation and capacity assumptions.

Reactor-by-reactor annual and cumulative storage requirements for the Middle Case are shown in Appendix A tables. Tables A.5 and A.6 show the additional annual and cumulative requirements for the maximum AR storage capacity scenario, while Tables A.7 and A.8 show the additional annual and cumulative requirements for the maximum AR storage capacity with intra-utility transshipment case. 
TABLE 11. Cumulative Additional Storage Requirements Maximum AR Storage

No New

Year Utility Data Orders Case Middle Case

1984

0

0

0

1985

1

0

0

1986

28

1987

119

1988

266

1989

459

18

46

155

303

20

1990

893

1991

1429

1861

2472

3276

483

866

1199

1647

2220

60

180

339

564

1993

1994

4086

2696

969

1995

1996

5362

3476

1311

1776

2368

2886

6613

4309

3696

4580 


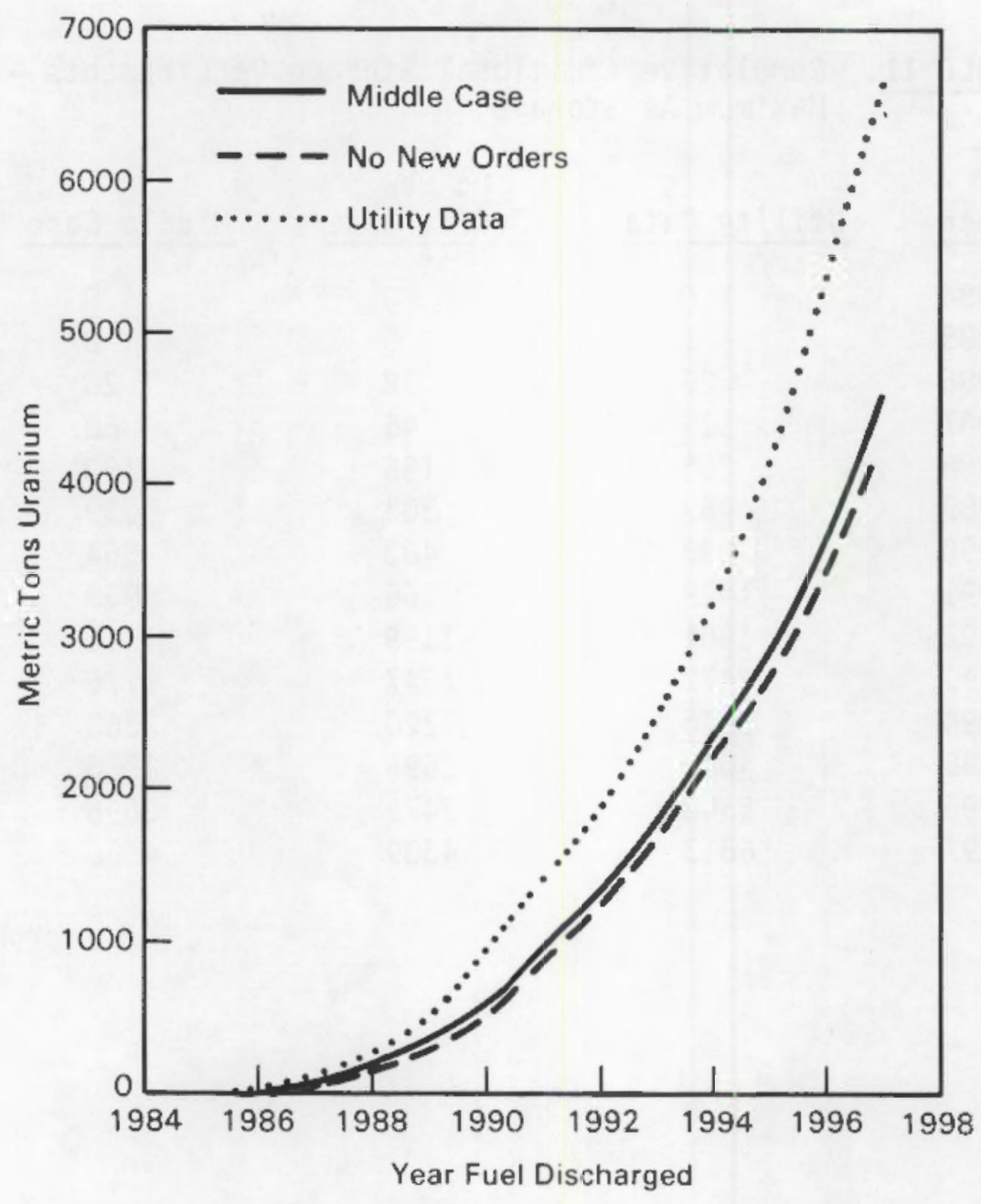

FIGURE 5. Projected Additional Cumulative Storage Requirements Maximum At-Reactor Storage Capacity: Adjusted Data Bases and Utility Projections 
TABLE 12. Cumulative Additional Storage Requirements (MTIHM) Maximum AR Storage Capacity Plus Transshipment

No New

Year Utility Base Orders Case Middle Case

1984

0

0

0

1985

0

0

1987

23

1988

1989

23

61

147

369

664

1047

1534

2231

3298

4533

0

0

5

5

34

44

121

299

500

878

1323

1870

2526

0

9

9

39

55

147

324

546

903

1323

1852

1997

4533

2592 


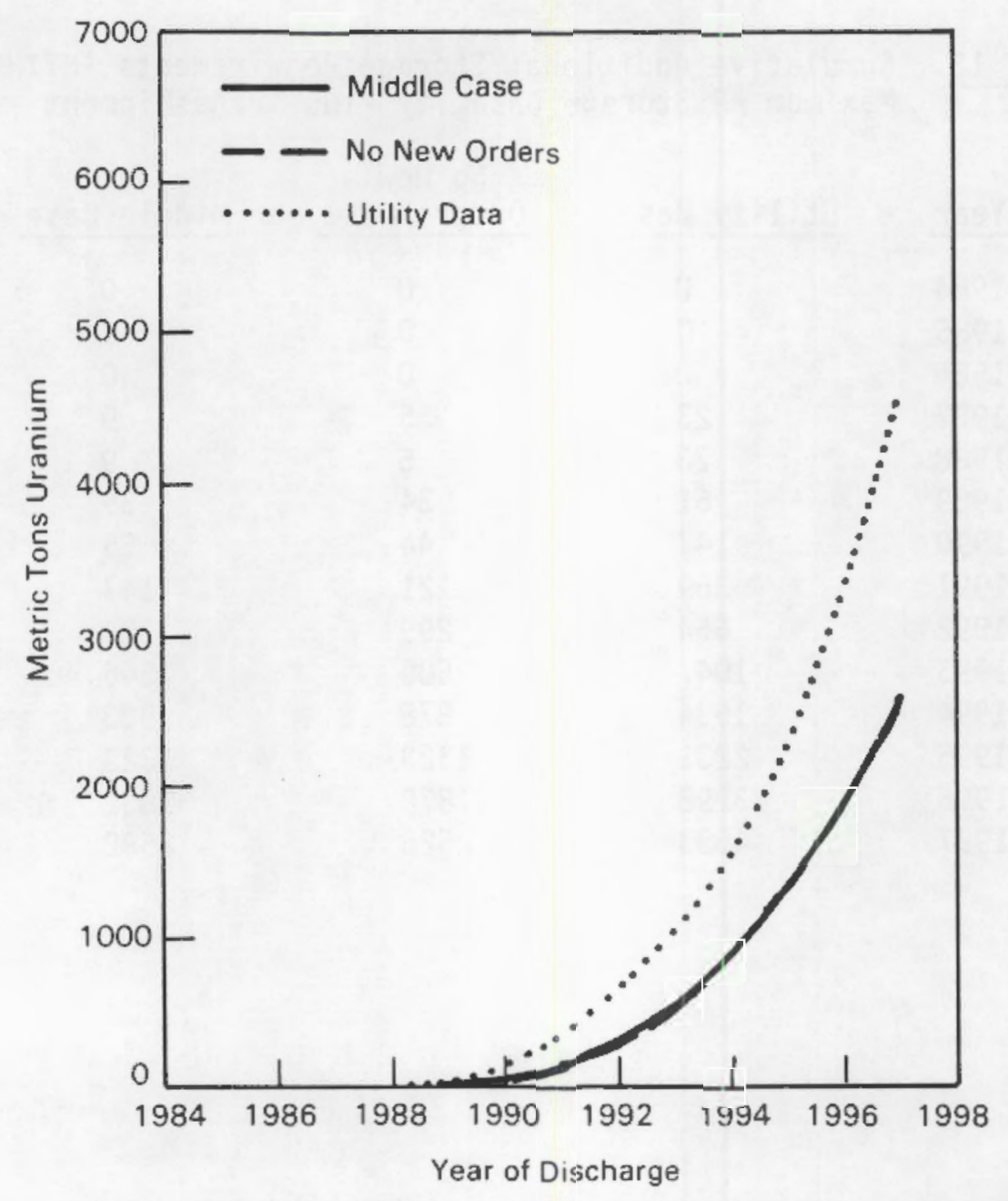

FIGURE 6. Projected Additional Cumulative Storage Requirements Maximum At-Reactor Storage Capacity Plus Transshipment: Adjusted Data Bases and Utility Projections 


\subsection{REFERENCES}

1. Gielecki, M. et al. 1984. Commercial Nuclear Power 1984: Prospects for the United States and the World. DOE/EIA-0438 (84), U.S. Department of Energy, Washington, D.C.

2. DOE. 1984. Spent Fuel Storage Requirements: An Update of DOE/RL-83-1. DOE/RL-84-1, U.S. Department of Energy, Richland Operations Office, Richland, Washington.

3. DOE. 1984. Spent Fuel and Radioactive Waste Inventory, Projections, and Characteristics. D0E/RW-0006, U.S. Department of Energy, Washington, D.C. 

APPENDIX A

DETAILED DATA BASE RESULTS 
APPENDIX A

DETAILED DATA BASE RESULTS

The following tables provide detailed results for all three scenarios: Utility, No New Orders, and Middle Case. The first two tables show comparisons between data base information, while the remaining tables supply reactorspecific information for the Middle Case. Similar reactor-specific information is furnished by the Spent Fuel Storage Requirements Report ${ }^{(2)}$ for the Utility Data Base.

Table Number Title

A.1 Startup and Shutdown Dates for Utility, No New Orders and Middle Cases

A.2 Startup and Shutdown Dates of Middle Case Generic Reactors

A.3 Middle Case 1983 Inventory and Projected Annual Reactor Discharges

A.4 Middle Case 1983 and Projected Inventories

A.5 Middle Case Maximum At-Reactor Capacity - Projected Annual Additional Storage Requirements

A.6 Middle Case Maximum At-Reactor Capacity - Projected Cumulative Storage Requirements

A.7 Middle Case Maximum At-Reactor Capacity - PIus Transshipment Projected Annual Storage Requirements

A.8 Middle Case Maximum At-Reactor Capacity - Plus Transshipment Projected Cumulative Storage Requirements 
TABLE A.1. Startup and Shutdown Dates for Utility, No New Orders, and Hiddle Cases




(contd) TABLE A.1.

\begin{tabular}{|c|c|c|c|c|c|c|}
\hline & Utilit & Case & Orders & ase & Middle & ase \\
\hline Reactor & Startup & Down & Startup & Down & Startup & Down \\
\hline INDIAN POINT-2 & $1974 / 07$ & 2006 & $1974 / 07$ & 2011 & $1974 / 07$ & 2006 \\
\hline BROWNS FERRY-1 & $1974 / 08$ & 2014 & $1974 / 08$ & 2012 & $1974 / 08$ & 2007 \\
\hline ZION-2 & $1974 / 09$ & 2008 & $1974 / 09$ & 2013 & $1974 / 09$ & 2007 \\
\hline OCONEE-2 & $1974 / 09$ & 2010 & $1974 / 09$ & 2012 & $1974 / 09$ & 2008 \\
\hline THREE MILE ISLAND-1 & $1974 / 09$ & 2008 & $1974 / 09$ & 2013 & $1974 / 09$ & 2008 \\
\hline ARKANSAS NUCL ONE -1 & $1974 / 12$ & 2014 & $1974 / 12$ & 2013 & $1974 / 12$ & 2008 \\
\hline PRAIRIE ISLAND-2 & $1974 / 12$ & 2008 & $1974 / 12$ & 2013 & $1974 / 12$ & 2008 \\
\hline PEACH BOTTOM-3 & $1974 / 12$ & 2008 & $1974 / 12$ & 2013 & $1974 / 12$ & 2008 \\
\hline OCONEE-3 & $1974 / 12$ & 2009 & $1974 / 12$ & 2012 & $1974 / 12$ & 2007 \\
\hline DUANE ARNOLD & $1975 / 02$ & 2010 & $1975 / 02$ & 2015 & $1975 / 02$ & 2010 \\
\hline BROWNS FERRY -2 & $1975 / 03$ & 2014 & $1975 / 03$ & 2012 & $1975 / 03$ & 2008 \\
\hline RANCHO SECO-1 & $1975 / 04$ & 2008 & $1975 / 04$ & 2013 & $1975 / 04$ & 2008 \\
\hline CALVERT CLIFFS-1 & $1975 / 05$ & 2009 & $1975 / 05$ & 2014 & $1975 / 05$ & 2009 \\
\hline FITZPATRICK & $1975 / 07$ & 2015 & $1975 / 07$ & 2015 & $1975 / 07$ & 2010 \\
\hline D C COOK-1 & $1975 / 08$ & 2009 & $1975 / 08$ & 2014 & $1975 / 08$ & 2009 \\
\hline MILLSTONE - 2 & $1975 / 12$ & 2010 & $1975 / 12$ & 2015 & $1975 / 12$ & 2010 \\
\hline HATCH-1 & $1975 / 12$ & 2016 & $1975 / 12$ & 2014 & $1975 / 12$ & 2009 \\
\hline BRUNSWICK-2 & $1976 / 05$ & 2010 & $1976 / 05$ & 2015 & $1976 / 05$ & 2010 \\
\hline BEAVER VALLEY-1 & $1976 / 05$ & 2010 & $1976 / 05$ & 2015 & $1976 / 05$ & 2010 \\
\hline TROJAN & $1976 / 05$ & 2015 & $1976 / 05$ & 2016 & $1976 / 05$ & 2011 \\
\hline INDIAN POINT-3 & $1976 / 08$ & 2015 & $1976 / 08$ & 2014 & $1976 / 08$ & 2009 \\
\hline ST. LUCIE-1 & $1976 / 12$ & 2010 & $1976 / 12$ & 2015 & $1976 / 12$ & 2010 \\
\hline BRUNSWICK-1 & $1977 / 03$ & 2010 & $1977 / 03$ & 2015 & $1977 / 03$ & 2010 \\
\hline BROWNS FERRY-3 & $1977 / 03$ & 2017 & $1977 / 03$ & 2013 & $1977 / 03$ & 2008 \\
\hline CRYSTAL RIVER-3 & $1977 / 03$ & 2017 & $1977 / 03$ & 2013 & $1977 / 03$ & 2008 \\
\hline CALVERT CLIFFS-2 & $1977 / 04$ & 2009 & $1977 / 04$ & 2014 & $1977 / 04$ & 2009 \\
\hline SALEM-1 & $1977 / 06$ & 2017 & $1977 / 06$ & 2013 & $1977 / 06$ & 2008 \\
\hline DAVIS-BESSE-1 & $1977 / 12$ & 2017 & $1977 / 12$ & 2016 & $1977 / 12$ & 2011 \\
\hline FARLEY-1 & $1977 / 12$ & 2012 & $1977 / 12$ & 2017 & $1977 / 12$ & 2012 \\
\hline NORTH ANNA-1 & $1978 / 06$ & 2011 & $1978 / 05$ & 2016 & $1978 / 06$ & 2011 \\
\hline D C COOK -2 & $1978 / 07$ & 2009 & $1978 / 07$ & 2014 & $1978 / 07$ & 2009 \\
\hline НАТСH-2 & $1979 / 09$ & 2019 & $1979 / 09$ & 2017 & $1979 / 09$ & 2012 \\
\hline ARKANSAS NUCL ONE-2 & $1980 / 03$ & 2020 & $1980 / 03$ & 2017 & $1980 / 03$ & 2012 \\
\hline NORTH ANNA-2 & $1980 / 12$ & 2011 & $1980 / 12$ & 2016 & $1980 / 12$ & 2011 \\
\hline FARLEY-2 & $1981 / 07$ & 2011 & $1981 / 07$ & 2017 & $1981 / 07$ & 2012 \\
\hline
\end{tabular}


(contd) TABLE A.1.

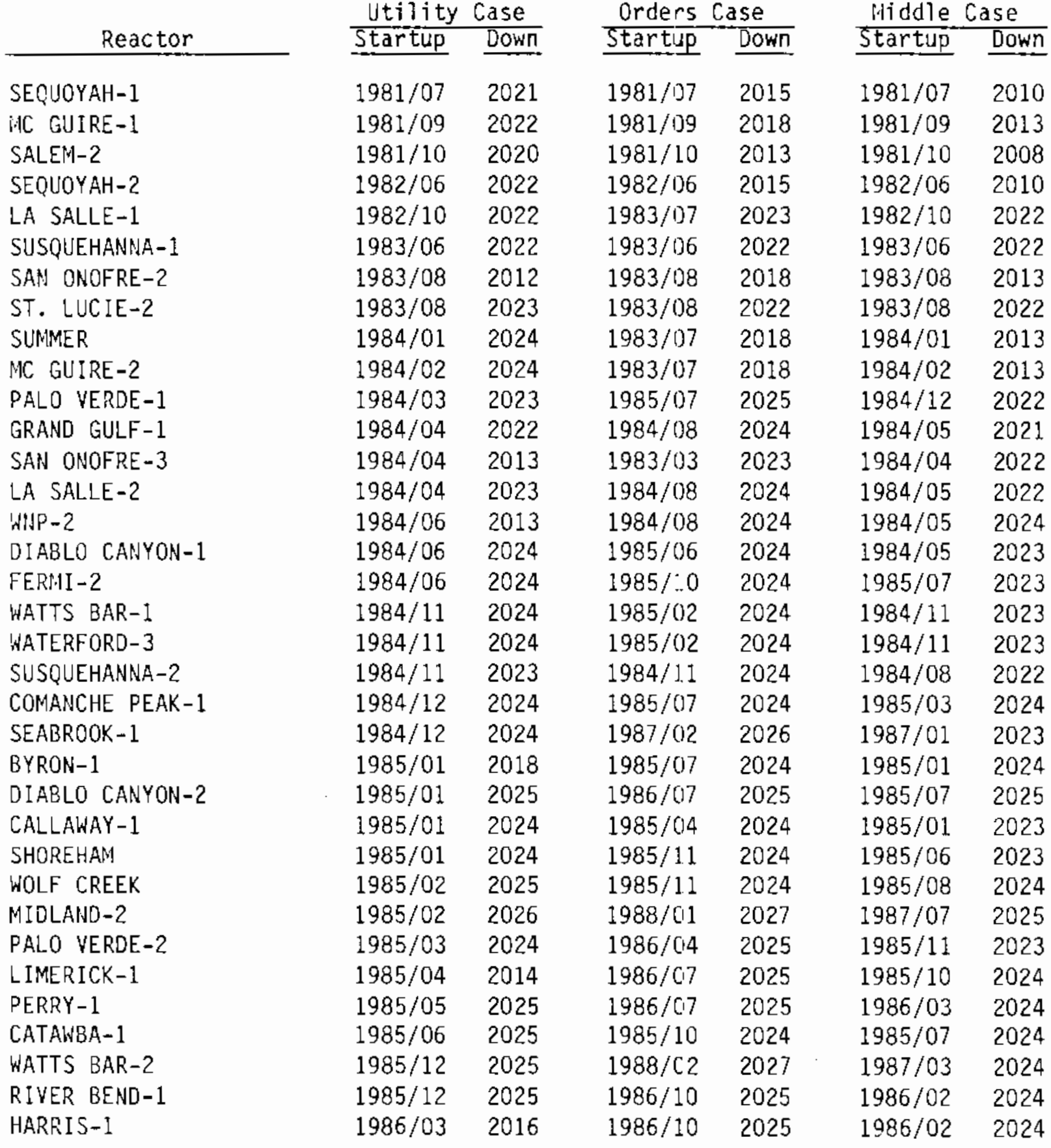


TABLE A.1. (contd)

\begin{tabular}{|c|c|c|c|c|c|c|}
\hline & Utility & Case & orders & ase & Middle & ase \\
\hline Reactor & Startup & Down & Startup & Down & Startup & Down \\
\hline HOPE CREEK-1 & $1986 / 03$ & 2027 & $1987 / 07$ & 2026 & $1986 / 12$ & 2026 \\
\hline BYRON-2 & $1986 / 04$ & 2019 & $1987 / 03$ & 2026 & $1986 / 07$ & 2024 \\
\hline BRAIDWOOD-1 & $1986 / 04$ & 2020 & $1987 / 04$ & 2026 & $1986 / 08$ & 2024 \\
\hline PALO VEROE-3 & $1986 / 05$ & 2026 & $1988 / 02$ & 2027 & $1987 / 07$ & 2025 \\
\hline BEAVER VALLEY-2 & $1986 / 05$ & 2025 & $1987 / 03$ & 2026 & $1986 / 07$ & 2024 \\
\hline MILLSTOHE-3 & $1986 / 05$ & 2014 & $1987 / 01$ & 2026 & $1986 / 07$ & 2024 \\
\hline COMANCHE PEAK-2 & $1986 / 06$ & 2025 & $1987 / 07$ & 2026 & $1986 / 09$ & 2024 \\
\hline ZIMNER-L & $1986 / 08$ & 2019 & & & & \\
\hline NINE MILE POINT-2 & $1986 / 10$ & 2026 & $1987 / 08$ & 2026 & $1987 / 01$ & 2025 \\
\hline CLINTON-1 & $1986 / 11$ & 2016 & $1987 / 07$ & 2026 & $1987 / 01$ & 2024 \\
\hline SEABROOK -2 & $1987 / 03$ & 2028 & & & $1992 / 01$ & 2027 \\
\hline VOGTLE-1 & $1987 / 03$ & 2027 & $1988 / 07$ & 2027 & $1987 / 08$ & 2026 \\
\hline MIOLAND-1 & & & & & $1988 / 07$ & 2023 \\
\hline BRAIDW000-2 & $1987 / 04$ & 2020 & $1990 / 11$ & 2029 & $1989 / 07$ & 2029 \\
\hline CATAWBA-2 & $1987 / 06$ & 2027 & $1988 / 10$ & 2027 & $1987 / 11$ & 2026 \\
\hline SOUTH TEXAS PROJ-1 & $1987 / 06$ & 2027 & $1988 / 12$ & 2027 & $1987 / 12$ & 2026 \\
\hline PERRY - 2 & $1988 / 05$ & 2028 & $1991 / 07$ & 2030 & $1990 / 05$ & 2027 \\
\hline WNP-3 & & & & & $1990 / 09$ & 2031 \\
\hline MARBLE HILL-1 & $1988 / 06$ & 2028 & & & & \\
\hline VOGTLE-2 & $1988 / 09$ & 2028 & & & $1991 / 11$ & 2027 \\
\hline GRAND GLLF-2 & $1988 / 12$ & 2028 & & & $1990 / 06$ & 2027 \\
\hline BELLEFONTE-1 & $1989 / 01$ & 2026 & $1989 / 10$ & 2028 & $1989 / 04$ & 2026 \\
\hline SOUTH TEXAS PROJ-2 & $1989 / 06$ & 2029 & & & $1991 / 08$ & 2028 \\
\hline LIMER ICK - 2 & $1990 / 04$ & 2015 & & & $1993 / 05$ & 2034 \\
\hline MARBLE HILL-2 & $1990 / 06$ & 2030 & & & & \\
\hline BELLEFONTE-2 & $1991 / 01$ & 2027 & $1993 / 02$ & 2029 & $1992 / 04$ & 2032 \\
\hline WNP - 1 & $1991 / 01$ & 2020 & & & $1992 / 04$ & 2032 \\
\hline HARTSVILLE-A1 & & & & & $1993 / 06$ & 2030 \\
\hline HARTSVILLE-A2 & & & & & $1996 / 12$ & 2030 \\
\hline YELLOW CREEK-1 & & & & & $1996 / 12$ & 2030 \\
\hline YELLOW CREEK-2 & & & & & $1997 / 12$ & 2030 \\
\hline CARROLL COUNTY-1 & $2004 / 06$ & 2039 & & & & \\
\hline CARROLL COUNTY-2 & $2005 / 06$ & 2040 & & & & \\
\hline
\end{tabular}


TABLE A.2. Startup and Shutdown Dates of Middle Case Generic Reactors

\begin{tabular}{|c|c|c|c|}
\hline Reactor Name (a) & Fed. Reg. & Startup & Shutdown \\
\hline P010201 & V & $2001 / 01$ & 2040 \\
\hline P010101 & IV & $2001 / 01$ & 2040 \\
\hline P010301 & IV & $2001 / 01$ & 2040 \\
\hline B010101 & I I I & $2001 / 01$ & 2040 \\
\hline B010205 & $V$ & $2001 / 05$ & 2041 \\
\hline P010410 & IV & $2001 / 10$ & 2041 \\
\hline P020201 & IX & $2002 / 01$ & 2041 \\
\hline P020101 & II & $2002 / 01$ & 2041 \\
\hline B020101 & IV & $2002 / 01$ & 2041 \\
\hline P020308 & $V$ & $2002 / 08$ & 2041 \\
\hline B020210 & VI & $2002 / 10$ & 2042 \\
\hline P030101 & I & $2003 / 01$ & 2042 \\
\hline P030201 & IV & $2003 / 01$ & 2042 \\
\hline 8030104 & III & $2003 / 04$ & 2043 \\
\hline P030305 & $V$ & $2003 / 05$ & 2042 \\
\hline$B 040101$ & IV & $2004 / 01$ & 2043 \\
\hline P040201 & II & $2004 / 01$ & 2043 \\
\hline P040101 & IV & $2004 / 01$ & 2043 \\
\hline P040310 & V & $2004 / 10$ & 2044 \\
\hline B050201 & V & $2005 / 01$ & 2044 \\
\hline B050101 & IV & $2005 / 01$ & 2044 \\
\hline P050301 & I I I & $2005 / 01$ & 2044 \\
\hline P050201 & $x$ & $2005 / 01$ & 2044 \\
\hline P050101 & IX & $2005 / 01$ & 2044 \\
\hline B050307 & $V I$ & $2005 / 07$ & 2045 \\
\hline P060201 & IV & $2006 / 01$ & 2045 \\
\hline P060101 & VII & $2006 / 01$ & 2045 \\
\hline P060305 & I & $2006 / 05$ & 2045 \\
\hline B060105 & $V$ & $2006 / 05$ & 2046 \\
\hline P070501 & V & $2007 / 01$ & 2046 \\
\hline P070401 & IX & $2007 / 01$ & 2046 \\
\hline P070201 & II & $2007 / 01$ & 2046 \\
\hline P070101 & IV & $2007 / 01$ & 2046 \\
\hline P070301 & III & $2007 / 01$ & 2046 \\
\hline B070101 & IV & $2007 / 01$ & 2046 \\
\hline
\end{tabular}


TABLE A.2. (contd)

\begin{tabular}{|c|c|c|c|}
\hline Reactor Name ${ }^{(a)}$ & Fed. Reg. & Startup & Shutdown \\
\hline $\mathrm{B} 070201$ & I I I & $2007 / 01$ & 2046 \\
\hline B070307 & $V$ & $2007 / 07$ & 2047 \\
\hline P070608 & VI & $2007 / 08$ & 2046 \\
\hline P080601 & $V$ & $2008 / 01$ & 2047 \\
\hline P080401 & I I & $2008 / 01$ & 2047 \\
\hline P080301 & I & $2008 / 01$ & 2047 \\
\hline P080201 & III & $2008 / 01$ & 2047 \\
\hline P080101 & IV & $2008 / 01$ & 2047 \\
\hline P080501 & IV & $2008 / 01$ & 2047 \\
\hline B080201 & $x$ & $2008 / 01$ & 2047 \\
\hline B080101 & IX & $2008 / 01$ & 2047 \\
\hline 8080302 & IV & $2008 / 02$ & 2047 \\
\hline P091301 & I & $2009 / 01$ & 2048 \\
\hline P091201 & I I I & $2009 / 01$ & 2048 \\
\hline P091101 & VI & $2009 / 01$ & 2048 \\
\hline P091001 & I & $2009 / 01$ & 2048 \\
\hline P090901 & IX & $2009 / 01$ & 2048 \\
\hline P090601 & VII & $2009 / 01$ & 2048 \\
\hline P090501 & $V I$ & $2009 / 01$ & 2048 \\
\hline P090401 & V & $2009 / 01$ & 2048 \\
\hline P090301 & IV & $2009 / 01$ & 2048 \\
\hline P090201 & III & $2009 / 01$ & 2048 \\
\hline P090101 & $v$ & $2009 / 01$ & 2048 \\
\hline P090801 & 11 & $2009 / 01$ & 2048 \\
\hline P090701 & IV & $2009 / 01$ & 2048 \\
\hline 8090201 & $V$ & $2009 / 01$ & 2048 \\
\hline 8090301 & IV & $2009 / 01$ & 2048 \\
\hline B090101 & IV & $2009 / 01$ & 2048 \\
\hline B090401 & II & $2009 / 01$ & 2048 \\
\hline 8090501 & III & $2009 / 01$ & 2048 \\
\hline B090601 & IV & $2009 / 01$ & 2048 \\
\hline B090705 & $V$ & $2009 / 05$ & 2049 \\
\hline P091408 & $V I$ & $2009 / 08$ & 2048 \\
\hline P100201 & $x$ & $2010 / 01$ & 2049 \\
\hline P100101 & $I X$ & $2010 / 01$ & 2049 \\
\hline P100503 & I II & $2010 / 01$ & 2049 \\
\hline
\end{tabular}


TABLE A.2. (contd)

\begin{tabular}{|c|c|c|c|}
\hline Reactor Name ${ }^{(a)}$ & Fed. Reg. & Startup & Shutdown \\
\hline P100301 & IV & $2010 / 01$ & 2049 \\
\hline P100401 & $v$ & $2010 / 01$ & 2049 \\
\hline B100101 & IV & $2010 / 01$ & 2049 \\
\hline B100201 & I & $2010 / 01$ & 2049 \\
\hline B100308 & $v$ & $2010 / 08$ & 2050 \\
\hline 8110101 & VII & $2011 / 01$ & 2050 \\
\hline 8110201 & IV & $2011 / 01$ & 2050 \\
\hline P110201 & II & $2011 / 01$ & 2050 \\
\hline P110301 & IX & $2011 / 01$ & 2050 \\
\hline P110401 & IV & $2011 / 01$ & 2050 \\
\hline P110501 & $v$ & $2011 / 01$ & 2050 \\
\hline P110601 & VI & $2011 / 01$ & 2050 \\
\hline P110101 & IV & $2011 / 01$ & 2050 \\
\hline B110302 & III & $2011 / 02$ & 2050 \\
\hline P110703 & V & $2011 / 03$ & 2050 \\
\hline P120201 & I & $2012 / 01$ & 2051 \\
\hline P120401 & IV & $2012 / 01$ & 2051 \\
\hline P120501 & $v$ & $2012 / 01$ & 2051 \\
\hline P120101 & IV & $2012 / 01$ & 2051 \\
\hline P120301 & II & $2012 / 01$ & 2051 \\
\hline B120201 & $x$ & $2012 / 01$ & 2051 \\
\hline B120101 & $I X$ & $2012 / 01$ & 2051 \\
\hline B120308 & III & $2012 / 08$ & 2052 \\
\hline P130301 & VI & $2013 / 01$ & 2052 \\
\hline P130201 & $v$ & $2013 / 31$ & 2052 \\
\hline P130101 & IV & $2013 / 101$ & 2052 \\
\hline P130401 & IV & $2013 / 01$ & 2052 \\
\hline B130101 & v & $2013 / 01$ & 2052 \\
\hline P130502 & IV & $2013 / 02$ & 2052 \\
\hline B130203 & II & $2013 / 03$ & 2053 \\
\hline P14050I & I & $2014 / 01$ & 2053 \\
\hline P140401 & v & $2014 / 01$ & 2053 \\
\hline P140301 & IV & $2014 / 01$ & 2053 \\
\hline P140201 & IX & $2014 / 01$ & 2053 \\
\hline P140101 & III & $2014 / 01$ & 2053 \\
\hline B140101 & IV & $2014 / 01$ & 2053 \\
\hline
\end{tabular}


TABLE A.2. (contd)

\begin{tabular}{|c|c|c|c|}
\hline Reactor Name $(a)$ & Fed. Reg. & Startup & Shutdown \\
\hline B140201 & $v$ & $2014 / 01$ & 2053 \\
\hline B140308 & VI & $2014 / 08$ & 2054 \\
\hline P140609 & VII & $2014 / 09$ & 2053 \\
\hline P150101 & IV & $2015 / 01$ & 2054 \\
\hline$P 150201$ & III & $2015 / 01$ & 2054 \\
\hline B150103 & I I & $2015 / 03$ & 2055 \\
\hline P150310 & IV & $2015 / 10$ & 2055 \\
\hline B160101 & IV & $2016 / 01$ & 2055 \\
\hline P160301 & $x$ & $2016 / 01$ & 2055 \\
\hline P160201 & IX & $2016 / 01$ & 2055 \\
\hline P160101 & $v$ & $2016 / 01$ & 2055 \\
\hline B160211 & $v$ & $2016 / 11$ & 2056 \\
\hline P170101 & II I & $2017 / 01$ & 2056 \\
\hline P170201 & IV & $2017 / 01$ & 2056 \\
\hline B170110 & I & $2017 / 10$ & 2057 \\
\hline P170311 & $v$ & $2017 / 11$ & 2057 \\
\hline P180101 & VI & $2018 / 01$ & 2057 \\
\hline P180203 & IV & $2018 / 03$ & 2057 \\
\hline B180106 & II & $2018 / 06$ & 2058 \\
\hline B190101 & V & $2019 / 01$ & 2058 \\
\hline P190201 & IV & $2019 / 01$ & 2058 \\
\hline P190101 & IX & $2019 / 01$ & 2058 \\
\hline P190305 & I I I & $2019 / 05$ & 2058 \\
\hline P200201 & V & $2020 / 01$ & 2059 \\
\hline P200101 & IV & $2020 / 01$ & 2059 \\
\hline B200101 & IV & $2020 / 01$ & 2059 \\
\hline P200309 & VII & $2020 / 09$ & 2059 \\
\hline B200209 & $x$ & $2020 / 09$ & 2060 \\
\hline
\end{tabular}

(a) The generic reactor name consists of four identification fields:

The first character denotes reactor type, $P$ for

PWR, B for BWR;

The next two characters are the last two digits

of the startup year;

The second set of two characters denote the sequence within a year;

The last two characters denote the startup month. 


\section{TABLE A.3. Middle Case 1983 Inventory and Projected Annual Reactor Discharges}

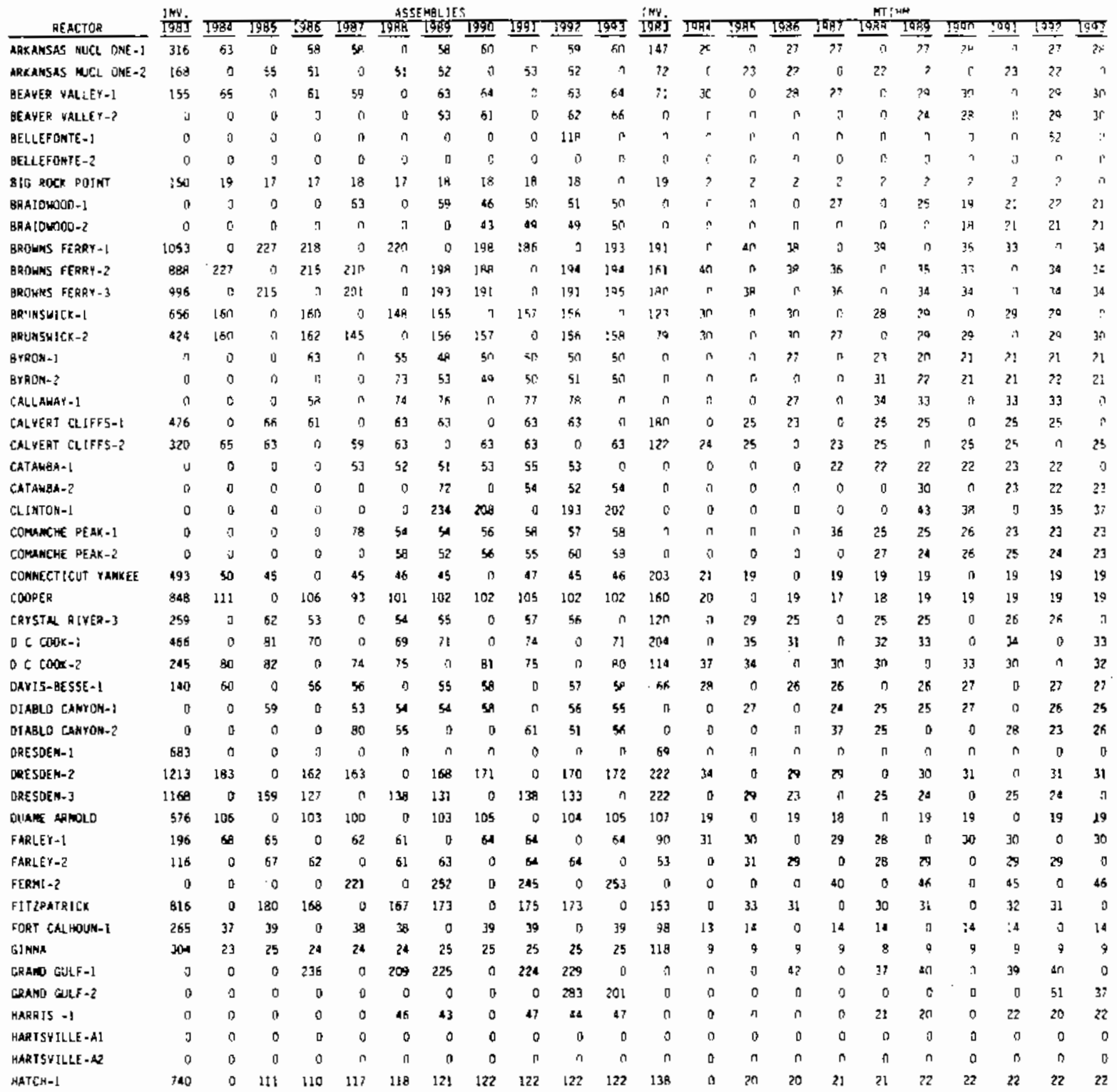


TABLE A.3. (contd)

\begin{tabular}{|c|c|c|c|c|c|c|c|c|c|c|c|c|c|c|c|c|c|c|c|c|c|c|}
\hline & NY. & & & & & & & & & & & [NY. & & & & & & & & & & \\
\hline REACTOR & 1983 & द्वत्तय & 1985 & 7986 & 1987 & 1938 & 1986 & T99\% & Iवका & 2942 & I993 & 1985 & D):4 & 1985 & 1986 & 987 & QRPA & Sक्व9 & $19 \$ 90$ & 991 & $199 ?$ & 97 \\
\hline$H A T C H=2$ & 520 & 102 & 121 & [1] & 118 & $11 \beta$ & 121 & 122 & 122 & 127 & $: 2 ?$ & 95 & 19 & 22 & 20 & 21 & 21 & 22 & $? ?$ & 22 & 27 & 22 \\
\hline HOPE CREEK-I & 0 & 0 & 0 & 0 & 0 & 155 & 0 & 228 & 230 & 0 & 233 & $\mathfrak{1}$ & 0 & $\mathrm{n}$ & n & 0 & $2 \mathrm{R}$ & $n$ & 41 & $4 z$ & $n$ & 42 \\
\hline UMOLDT gay & 250 & 194 & 0 & n & 0 & n & ה & 0 & $n$ & $B$ & п & th & 13 & $\pi$ & $n$ & $\Pi$ & $n$ & $n$ & $n$ & n & .7 & \\
\hline IADCLAN POL & 160 & 0 & 0 & 0 & 0 & 0 & 0 & 0 & 0 & fी & n & 3I & ก & 0 & 0 & n & n & 0 & 0 & 0 & 0 & \\
\hline 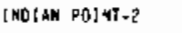 & 300 & 61 & 61 & 0 & 60 & 62 & $\pi$ & 63 & 6.3 & $n$ & 6.7 & 139 & $2 R$ & 29 & $n$ & 27 & $? R$ & 0 & 29 & 29 & nִ & 29 \\
\hline LNOLIAN POIYT-3 & 216 & 0 & $x$ & 0 & काI & 0 & 65 & 87 & 0 & the & $n$ & 99 & ก & 30 & n & 27 & 0 & pos & 3 & 0 & 30 & \\
\hline XEWAUNEE & $3 \infty 0$ & 35 & 33 & 33 & 32 & 33 & 33 & 33 & 33 & 33 & 33 & 118 & 13 & 13 & {$[3$} & 12 & 13 & 13 & 13 & 13 & 9 & 13 \\
\hline LA DOSSE & 213 & 21 & 21 & 20 & 20 & 21 & 21 & 21 & 21 & 21 & 22 & 25 & ? & 2 & 2 & ? & 2 & 2 & 2 & 2 & 2 & . \\
\hline LA SALLE-1 & 0 & 0 & 193 & 0 & 186 & 176 & 0 & t.84 & 205 & 0 & 195 & n & 0 & 35 & 0 & 34 & 32 & $n$ & Is & 37 & $n$ & 36 \\
\hline LA SALLE-2 & 0 & 0 & 0 & ta2 & 191 & 174 & 0 & 198 & 207 & 0 & 196 & 0 & 0 & (1) & 33 & 35 & 32 & 0 & 34 & 19 & 0 & 36 \\
\hline IMER TCK-i & 0 & 0 & 0 & 149 & 0 & 204 & 200 & i) & 232 & 212 & ก & t & 0 & 0 & 27 & ↔ & 40 & 39 & $n$ & 42 & 39 & \\
\hline I IERICK $R$ & 0 & 0 & 0 & 0 & 0 & 0 & 0 & 0 & 0 & 0 & 0 & 0 & 0 & $\square$ & 0 & 0 & 0 & 0 & 0 & 0 & j & \\
\hline MAIME YAAKEEE & 649 & 66 & 63 & 0 & 51 & 61 & 0 & 63 & 63 & 0 & 63 & 245 & 29 & 24 & 0 & 23 & 23 & 0 & 24 & 24 & $j$ & 34 \\
\hline : 6 & 29 & 50 & 51 & 55 & 49 & 55 & 52 & 54 & 0 & 55 & 53 & 14 & 23 & 23 & 25 & 21 & 23 & 22 & 23 & j & 23 & 22 \\
\hline AC GLIRE-Z & 0 & 0 & 50 & 58 & 51 & 53 & SA & 0 & 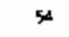 & 53 & $\$ 5$ & 0 & 0 & 23 & 26 & 23 & 23 & 23 & D & 23 & 27 & 23 \\
\hline 11OLAND.1 & 0 & 0 & 0 & 0 & 3 & 0 & 0 & 0 & 52 & 0 & 54 & 0 & t. & 0 & 0 & 0 & 0 & 0 & D & 24 & 0 & 25 \\
\hline MIDLAND - Z & 0 & 0 & 0 & $n$ & i) & Q & 0 & 50 & 55 & חִ & 63 & $n$ & $n$ & $B$ & $n$ & $n$ & $n$ & 0 & 23 & 26 & 0 & 29 \\
\hline MILLSTONE.I & 1136 & 172 & 0 & 178 & 267 & 0 & 172 & 0 & 173 & G & 176 & 215 & 31 & 0 & 31 & 29 & 0 & 30 & ก & נז & 7 & ו \\
\hline HLLSTOME-2 & 376 & 0 & 73 & 51 & 59 & 69 & $n$ & $\pi n$ & 66 & ก.ร & $n$ & 145 & n & 29 & 21 & 24 & 29 & 0 & $? ?_{R}$ & 27 & 26 & \\
\hline MILISTONE - 3 & 0 & 0 & 0 & 0 & 0 & 57 & 59 & 52 & $s R$ & 57 & 56 & 0 & 0 & 0 & n & $n$ & 26 & 27 & 24 & 27 & 25 & 26 \\
\hline ONTICELLE & 1016 & 100 & 102 & $8 n$ & B. & 91 & 89 & 9? & 91 & वा & $n$ & 192 & 19 & เ9 & ] & 15 & 16 & 26 & la & $1^{k}$ & in & \\
\hline NINE MILE DOTNT-I & 1044 & 158 & 0 & 159 & 0 & 168 & 0 & 175 & i) & 179 & 8 & 199 & $\infty$ & $B$ & $3 n$ & $n$ & $3 n$ & 0 & 31 & 9 & \pm & ( \\
\hline NIM MILE SOINT-? & 3 & 0 & n & n & $n$ & $n$ & 7 & חי & 233 & $n$ & 292 & ה & $n$ & $\uparrow$ & $n$ & r. & $n$ & 0 & 41 & $\&$ & $n$ & 4: \\
\hline MORTH AKGMA-1 & 193 & 6) & $\mathbf{S 8}$ & $n$ & 58 & 59 & $n$ & 51 & $\sin$ & 0 & 69 & pa & 31 & $\eta ?$ & n & $2 \hat{z}$ & $2 \%$ & $n$ & 29 & 78 & $\pi$ & $2 ?$ \\
\hline NORTH ANEL-? & $: n$ & 59 & n & 斻 & $5 p$ & ? & so & क] & $n$ & fi & $6 !$ & 51 & 27 & $\Pi$ & 27 & 27 & $n$ & 27 & ta & 0 & $2 \AA$ & ? $\mathrm{H}$ \\
\hline JCONEE - ! & 677 & 0 & 57 & 55 & $\pi$ & $\$ 7$ & ss & 0 & 55 & 0 & $5 R$ & 315 & 0 & 76 & 25 & ก & 26 & 25 & .7 & 25 & c. & 2 \\
\hline OCONEE - 2 & 392 & 0 & 6n & 0 & 59 & $5+4$ & $n$ & 50 & $\pi$ & Ko & sn & $1 \mathrm{~B} 2$ & ก & $2 R$ & ก & 23 & $2 !$ & 0 & 29 & 7 & 78 & 28 \\
\hline PCLUEE - ? & 56 & 70 & st & 0 & Sit & 0 & 60 & 60 & 3 & 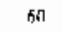 & 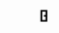 & 26 & 32 & 25 & 0 & 27 & $n$ & 29 & 28 & 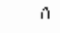 & 22 & \\
\hline DYSTER EREEK & 980 & 0 & $16 ?$ & 0 & J 6 G & 147 & 0 & $i n$ & $n$ & 178 & 0 & 192 & ה & 29 & $n$ & 29 & 26 & J] & 31 & $r$ & 31 & \\
\hline PAL I SAUES S & 47 & 0 & 62 & 9 & 57 & $5 x$ & 0 & 60 & 59 & 0 & fin & 194 & 0 & 24 & 1 & 22 & 23 & $n$ & 23 & 23 & $n$ & 23 \\
\hline PALO VERGE -: & 0 & .7 & 0 & 0 & $B 3$ & 53 & 55 & s7 & $S R$ & 56 & 57 & $n$ & $n$ & .7 & $n$ & 35 & 25 & 22 & 24 & 25 & 24 & $?$ \\
\hline OALO VERDE -? & 0 & v & 0 & 0 & 0 & 39 & 62 & 57 & $\$ 7$ & 56 & $5 \mathrm{~h}$ & $n$ & : & $n$ & 0 & 0 & 37 & 25 & 23 & 24 & 2 & 2 \\
\hline PALO YERDE - ] & ป & c & 0 & ד & 0 & 69 & fad & 57 & $5 ?$ & $5 ?$ & 4 & $n$ & $n$ & $B$ & ก & $r$ & 70 & 25 & 23 & 24 & 25 & 2 \\
\hline PEACH DT'DM-Z & 1170 & 260 & 0 & 252 & 0 & $2 ! 3$ & 0 & 220 & 221 & 0 & 222 & 213 & به & $\jmath$ & Af & 7 & 30 & 0 & 40 & 40 & כ & : \\
\hline PEALH BOT OM-3 & 1212 & 0 & 255 & п & 211 & 209 & in & 223 & 227 & $n$ & $m 3$ & 22! & $n$ & $\Delta F$ & ד & $3 R$ & $3 R$ & 0 & si & 4] & $n$ & $4:$ \\
\hline PERR - I & 0 & .7 & (8) & 0 & 0 & 203 & $24 B$ & ח & 352 & 755 & ר? & $n$ & $n$ & ר & $n$ & n & 17 & 44 & $n$ & $\Delta \hat{~}$ & $2 \pi$ & \\
\hline PERQY $=2$ & 0 & 0 & n & $n$ & 7 & r. & $n$ & 1 & $r$ & 590 & 785 & $n$ & a & $n$ & $r$ & $n$ & $r$ & $n$ & $n$ & 1 & 29 & 35 \\
\hline D L LCALERTI & 1004 & it & 0 & lot & 158 & 0 & :57 & $r$ & IFR & $n$ & 165 & ian & $n$ & ? & $3 n$ & 28 & $n$ & रก & 9 & jn & 7 & ? \\
\hline $3 \cap[N T$ AEACH $=1$ & $23 k$ & 9 & 35 & $3 n$ & $3 \pi$ & 31 & 32 & 32 & 37 & ?י & 17 & S4 & $n$ & 14 & 17 & 37 & 17 & $! 1$ & 11 & $! !$ & $!$ & 1) \\
\hline DOINT EEACH-2 & 244 & 39 & 28 & 26 & 28 & 27 & 29 & 28 & 28 & $2 \mathrm{R}$ & $>9$ & प9 & 16 & 11 & : & 11 & ln & $1 !$ & !n & $1 n$ & $! n$ & \\
\hline PQA[R]E TSLAND-] & 300 & 19 & 35 & 35 & 37 & 35 & ja & 36 & 16 & 34 & 36 & $: ; n$ & 2 & 14 & $\vdots 4$ & 17 & 14 & 14 & $: 4$ & is & 34 & $\vdots$ \\
\hline PQALR:E [SLLND-2 & 281 & 32 & 35 & 33 & 35 & n & 35 & 36 & 35 & .15 & 39 & 112 & is & 14 & 13 & 14 & r: & 19 & 14 & 14 & $1=$ & is \\
\hline 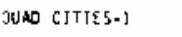 & $: 013$ & 183 & I65 & 0 & 182 & 155 & 0 & 15.4 & 15.3 & $n$ & $15 ?$ & $19 ?$ & $\exists ?$ & 20 & 1 & $3 ?$ & 97 & n & 97 & $3 ?$ & $r:$ & 7 \\
\hline JUAD CITIES-2 & 1119 & 0 & 159 & 100 & 0 & 145 & 145 & 0 & 143 & $14 \mathrm{~h}$ & 0 & 213 & n & 28 & is & $n$ & if & $2 \pi$ & הי & 25 & 26 & \\
\hline RAM[H] $\$ E: 0]$. & 260 & 51 & 49 & $n$ & $\Delta 4$ & 4.8 & 51 & sn & 0 & 49 & 49 & 121 & 24 & 33 & $n$ & $7 n$ & 27 & 24 & $2 ?$ & 0 & $? 2$ & 23 \\
\hline RIYER BENO-1 & 0 & 0 & 0 & u & 118 & 140 & 130 & 127 & $14]$ & 129 & 137 & 0 & 0 & 0 & 0 & $2 i$ & 26 & 23 & 23 & 25 & 24 & 2 \\
\hline ROBI YSON- $\hat{\mathrm{L}}$ & 157 & 50 & a & d6 & 4) & 43 & $n$ & 45 & 46 & 45 & $\Delta$ & 68 & 26 & I] & $7 n$ & $1 \mathrm{~A}$ & $m$ & ก & 19 & 211 & 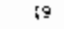 & \\
\hline ZRLÉM-1 & 212 & $8 \mathrm{i}$ & 13 & 0 & ני & 66 & 0 & 64 & 65 & in & Es & 97 & II & 34 & t: & 33 & 30 & 0 & 30 & 30 & 0 & \\
\hline
\end{tabular}


TABLE A.3. (contd)

\begin{tabular}{|c|c|c|c|c|c|c|c|c|c|c|c|c|c|c|c|c|c|c|c|c|c|c|}
\hline & $m$. & & & & & & noLIE & & & & & I צy. & & & & & & & & & & \\
\hline REACTOR & 19835 & 1982 & I\$35 & 7986 & 19837 & 15BG & .989 & 1990 & I\$9! & Tag? & 1945 & FमS & 19:3 & T0R5 & उुक्ष & T987 & 9915 & ग9स9 & T990 & T99? & 1992 & 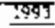 \\
\hline SALEM-2 & 72 & 0 & 80 & 65 & 53 & 0 & 62 & "1 & of & 3 & 75 & $3]$ & 0 & 37 & 3 & 45 & 0 & $\$ 3$ & 35 & 1 & 34 & 39 \\
\hline SAN DMOFRE-1 & 94 & J & 0 & 38 & ] & 10 & 0 & 46 & 0 & 46 & '] & 15 & 0 & J & 14 & 0 & 15 & i) & $: ?$ & J & 17 & 0 \\
\hline SAN JMOERE-2 & 了 & $5 ?$ & 0 & it & 67 & D) & 0 & 0 & 69 & 9 & $T_{1}$ & ] & 24 & 0 & 29 & 28 & a & 30 & 门 & 29 & $n$ & 30 \\
\hline SAN ONOFRE-3 & 0 & 0 & 55 & 70 & 0 & bj & i] & 74 & is & 68 & j & 0 & 0 & 23 & 28 & $\pi$ & 26 & 1) & 32 & 了 & 29 & 0 \\
\hline SEABROOK $=1$ & Ј & $n$ & 0 & ] & D & 56 & 54 & 56 & 55 & 57 & 56 & J & 1) & 0 & 0 & 1) & 24 & 23 & 24 & ? & 24 & 24 \\
\hline SEABRCOX - 2 & 0 & 0 & 0 & o) & D & 0 & 0 & 0 & 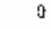 & . & 1) & b & 0 & 0 & :1 & 0 & 7 & 1 & :] & 0 & 1) & I \\
\hline SEQUOYAH-L & 140 & 0 & 63 & 12 & 0 & 12 & 0 & 73 & i3 & a & 74 & 62 & ? & 29 & 27 & 0 & 32 & $n$ & 32 & 32 & ] & 33 \\
\hline SEQUOHAH-2 & $\Delta a$ & 66 & 57 & D & 65 & 0 & 14 & 13 & 0 & 33 & 74 & 31 & to & 30 & ] & 29 & 0 & 33 & 32 & $\Rightarrow$ & 32 & 33 \\
\hline \$HOREHAN & 0 & 0 & 0 & 0 & 0 & 248 & $1 \leq B$ & D & 135 & 142 & 0 & $n$ & n & 0 & 0 & ป & 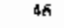 & 29 & $\mathrm{n}$ & 25 & 26 & I \\
\hline SOUTH TEXAS PROS-I & 0 & D & D & 0 & 0 & 0 & 63 & 60 & so & 55 & 96 & 0 & B & 0 & a & 0 & 0 & 34 & $\$ 2$ & 21 & 30 & 3n \\
\hline SOUTH TEXAS PRQN-2 & ') & ก & 0 & 0 & is & $\pi$ & n & 1 & D & 49 & 5 月 & $n$ & n & $n$ & $n$ & : & $n$ & $n$ & n & 1 & 76 & 31 \\
\hline ST. \UCIE-L & 364 & 0 & $\mathrm{~J}$ & 35 & 55 & $\pi$ & $r_{2}$ & 70 & 0 & 73 & !! & 140 & $n$ & $n$ & 32 & 23 & 0 & $2 \pi$ & 26 & נ. & 27 & 26 \\
\hline ST, LUCIE-2 & 0 & $\mathrm{~J}$ & i) & Tg & 69 & 0 & a7 & 75 & 0 & $\$$ & 17 & $n$ & 1 & ה & 30 & 29 & i & 25 & 29 & : & 26 & 9 \\
\hline 5UMAR $R$ & 1) & 0 & 0 & 0 & 56 & 59 & 0 & fil & $5 ?$ & 1) & fit & $\mathrm{a}$ & $\omega$ & 0 & 1] & 23 & 26 & $\exists$ & 27 & (3) & n & 28 \\
\hline SURRY - -6 & 398 & 51 & n & 49 & 36 & 0 & $6+8$ & sa & 0 & 53 & 54 & 180 & 23 & o & 22 & 16 & 3 & 31 & 26 & 1 & 24 & 25 \\
\hline SURRY $Y=2$ & 227 & 0 & 54 & 52 & 0 & 51 & 53 & ) & 53 & sa & 0 & 104 & if & 25 & 24 & 0 & 23 & 24 & $\mathrm{j}$ & 24 & 25 & is \\
\hline ESOSUEHANMA - 1 & 0 & $16 !$ & 206 & 0 & 202 & 210 & g & 223 & PIR & a & 220 & 0 & 99 & 59 & n & 37 & 40 & 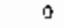 & 39 & IA & 7 & 39 \\
\hline SLLSOUEHANAA +2 & 0 & 3 & 0 & 225 & 195 & $a$ & 211 & 215 & $\mathrm{~J}$ & 219 & 221 & 0 & 0 & o & 11 & 36 & ) & 39 & 38 & 9 & 19 & 39 \\
\hline THREE MILE ISLAWO-1 & 200 & 0 & 45 & 64 & J & 53 & 66 & 0 & 48 & 66 & a & 97 & $\tau$ & $2 t$ & ?0 & 7 & 29 & 31 & D & 34 & 31 & ] \\
\hline TROJAKH & 260 & 50 & 18 & 12 & i & $\mathbb{4} 0$ & ง & 42 & $\$ 2$ & 42 & $\$ 2$ & 120 & 23 & 18 & 19 & 19 & 19 & 19 & 19 & 19 & 19 & is \\
\hline TURKEY POLMT-3 & 369 & u & 55 & นด & $\mathrm{u}$ & 54 & 49 & 0 & 56 & 49 & ] & 167 & 0 & 25 & 22 & J & 25 & 22 & D & 26 & 32 & ] \\
\hline TURKEY POTMT -4 & 314 & 60 & 58 & 0 & 53 & 55 & 0 & 55 & 57 & $n$ & 55 & 143 & 27 & 26 & ) & 24 & 25 & 0 & 25 & 26 & 1) & 25 \\
\hline VERMONT YATKEE & 1098 & 4 & 83 & 0 & 28 & 71 & 80 & 81 & so & 90 & A1 & $2 \infty$ & 19 & :5 & n & : & 13 & 15 & 15 & 15 & $: 5$ & $: 5$ \\
\hline NOGTLE-I & 0 & 1) & 0 & u & ] & a) & 57 & 55 & 55 & 55 & 57 & ) & 0 & 0 & 0 & 12 & $j$ & 25 & 25 & 25 & 25 & 26 \\
\hline YOGTLE-? & 18 & D) & 3 & 1 & ] & $\mathrm{n}$ & $\cdot]$ & 11 & $n$ & '] & 58 & $n$ & . & s & B & 1 & a & $1)$ & $n$ & $\pi$ & $\mathrm{J}$ & 27 \\
\hline 'HATEAFORO-3 & 0 & 0 & 0 & 61 & 5) & 49 & bi & 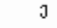 & $x_{2}$ & 57 & א2 & 0 & $\mathrm{~J}$ & 0 & $\eta$ & 27 & 20 & 28 & J & 26 & 29 & 26 \\
\hline$\# A T T S$ 3AR $=1$ & J & ] & 0 & 57 & 0 & $\infty 0$ & 63 & i] & 63 & !3 & ד & $n$ & 7 & $\pi$ & 26 & n & $? 7$ & 28 & $n$ & 31 & 12 & a \\
\hline AATTS 3AA-2 & 1" & 3 & 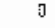 & .7 & '] & $n$ & 5 a & 50 & 0 & 63 & ח & i & 0 & $\pi$ & 0 & n & i" & 25 & 21 & 7 & 28 & $3 \mathrm{l}$ \\
\hline AIP - ! & 1 & $n$ & nִ & $n$ & $\mathrm{a}$ & ? & 1 & $n$ & n & 1 & ה & " & $\pi$ & וי & n & .1 & 1 & $n$ & a & $\rightarrow$ & $n$ & ? \\
\hline 'LNP - 2 & $n$ & 1 & 151 & 143 & 144 & 138 & 157 & 147 & 106 & i: 16 & 148 & $n$ & 7 & $7 t$ & 26 & 25 & 24 & 28 & 27 & 27 & $? ?$ & 27 \\
\hline hNP - $]$ & $\cdot 1$ & it & ] & 10 & n & $\mathrm{n}$ & 1 & $n$ & 1 & 74 & ร9 & 1 & $n$ & $n$ & it & $\mathrm{n}$ & 1 & 7 & $n$ & 3 & 11 & $?$ \\
\hline mILFF TREEK & 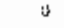 & J & J & 1 & 0 & 55 & 59 & ? & $\hat{n} 2$ & 63 & $?$ & )) & 3 & ו' & " & n & 25 & 27 & 1 & 29 & 39 & $n$ \\
\hline 'ATREEE & 265 & 38 & 32 & 13 & ]3 & ]: & 0 & is & 37 & 35 & $\pi$ & 6.3 & 4 & $\mathrm{~T}$ & $\mathrm{~s}$ & a & ? & $n$ & 2 & $?$ & ; & n \\
\hline PELLL'W CREEK=1 & ? & :] & . & $n$ & 0 & 1) & j & ) & $1)$ & J & J & .) & $\mathrm{J}$ & i & !) & 1) & ๆ & 1 & 7 & ) & $1)$ & $\pi$ \\
\hline 'ELLOW AREEK-2 & 0 & 0 & 11 & 0 & 10 & $\pi$ & i) & i) & 1 & 1] & וי & ii & n & 7 & $\pi$ & 1 & 1 & 19 & $n$ & 1 & .1 & $?$ \\
\hline$\because: U^{M}-1$ & 237 & 47 & jo & 10 & $\$ 2$ & $\$ 2$ & 42 & 12 & $\$ 2$ & 42 & 43 & 200 & 21 & 23 & It & $\vdots 9$ & $: 9$ & 19 & 19 & 19 & 19 & ?ח \\
\hline $2: i n-2$ & 154 & 37 & 沙 & $\$ 2$ & 4d & 4t & 42 & $\$ 2$ & 42 & 42 & 42 & 164 & 26 & $? 3$ & 19 & เด & 19 & 19 & 19 & 17 & 9 & $! 9$ \\
\hline MRUUKSWICX-i PWR PUOL & 160 & 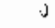 & ] & נ] & 0 & $\therefore$ & ] & a) & 2 & J & 1 & $i$ & 3 & a & 1) & it & $\mathrm{J}$ & 3 & 1) & .) & i) & ] \\
\hline JRI,A5W[CX-2 PhR POUL & 144 & $u$ & 0 & 0 & 13 & ו & $n$ & 1) & ? & u & 1) & ń6 & 0 & 1 & 1 & 1 & :7 & i) & 1) & $i$ & 1 & 1) \\
\hline 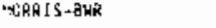 & ;s & $\mathrm{J}$ & J & 1] & o & 1) & .] & ] & it & $\mathrm{J}$ & $\mathrm{J}$ & 145 & .] & ו. & 1) & 0 & 0 & 3 & J & ) & 1) & as \\
\hline 'ALAR [S-PLTR & 154 & 1) & 0 & (1) & $\because$ & 1 & D & 0 & י. & 0 & 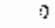 & $: 77$ & 13 & $n$ & t & די & 1 & 0 & $n$ & : & j] & J \\
\hline 'EEST VALLER-BUR & +18 & $\mathrm{~J}$ & 1 & 0 & 1) & 1) & J & 1) & 1) & J & ] & at & 7 & 1 & ני & i) & ] & J & 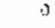 & . & 0 & 0 \\
\hline ZEST FALLEY-PYR & 235 & 0 & 1) & 1) & 13 & .J & 0 & $n$ & 7 & 1 & ה & 7.3 & ? & " & is &. & 0 & $n$ & 1) & 1 & .] & i] \\
\hline 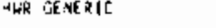 & $j$ & '] & .1 & i) & 0 & ") & j & 0 & is & $\mathrm{J}$ & ') & 3 & 1] & 1 & $n$ & B & 3 & J & י & ) & וי & $\mathrm{J}$ \\
\hline PHR GE YERTIC & 1) & 1) & 1) & n & ip & 1 & $\mathrm{n}$ & 0 & i) & 1) & 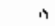 & 1 & n & 1 & ॥ & 1 & 1 & i & 13 & I & $n$ & 0 \\
\hline
\end{tabular}


TABLE A.3. (contd)

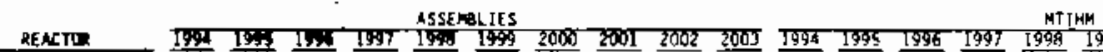

\begin{tabular}{|c|c|c|c|c|c|c|c|c|c|c|c|c|c|c|c|c|c|c|c|c|}
\hline & & & & & 9 & 99 & 0000 & 01 & 002 & 003 & $\frac{94}{94}$ & $99 \underline{\underline{9}}$ & उ96 & 1967 & 998 & 1996 & 000 & 2001 & कीQ? & $0 \pi 05$ \\
\hline axpuses act and & $a$ & 6 & $\omega$ & 0 & 62 & 63 & 0 & $6]$ & 62 & (1) & 0 & 28 & 28 & ה & 29 & $\pi$ & $\Pi$ & 29 & 29 & 0 \\
\hline Aekrenses and om-2 & 53 & sa & 0 & se & 55 & ס & 56 & 36 & D & 55 & 23 & 33 & 0 & 23 & 23 & D & 24 & 24 & $\therefore$ & $2 ?$ \\
\hline QEAVER VRLLCY-I & 0 & $M$ & SS & 0 & 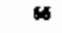 & 69 & $a$ & 68 & 86 & 0 & D & 30 & 30 & ? & 3) & 31 & 0 & 31 & 30 & 0 \\
\hline BEAYER YNLLEY-2 & 0 & 65 & 0 & 65 & $\alpha$ & 0 & 69 & 6 म & 0 & $6 \hbar$ & $a$ & 31 & 0 & $3 n$ & $3 n$ & 0 & 31 & 3] & יד & $3 n$ \\
\hline BELLEFONIE-] & I & 83 & 38 & 0 & 59 & 0 & 89 & Bq & 0 & 87 & 34 & 37 & 39 & ก & 39 & n & 39 & 39 & 0 & $3 R$ \\
\hline DELLLF ONTE-? & 52 & 72 & $\pi$ & 87 & $a$ & 89 & 90 & 0 & $8 B$ & 87 & 29 & 31 & 1 & 38 & ก & 39 & an & $n$ & 39 & 39 \\
\hline IIG AIICK DOSNT & 18 & 18 & 18 & 18 & 19 & 19 & BA & $n$ & 0 & 0 & 2 & 2 & 2 & 2 & 2 & 2 & 11 & 0 & 0 & 0 \\
\hline RANOW000-1 & 51 & $\$ 1$ & 51 & 52 & 53 & 53 & 53 & 53 & 52 & 52 & 22 & 22 & 27 & 22 & 22 & 77 & $? 7$ & 27 & 22 & 77 \\
\hline $\operatorname{arnc} 10 \mathrm{~h} 000-2$ & 51 & 51 & 52 & 52 & 53 & 53 & 53 & 53 & 52 & 52 & 22 & 22 & 22 & 22 & 22 & 22 & 22 & 22 & 22 & 22 \\
\hline IROWIE FERRY-I & 197 & 0 & 197 & 199 & 0 & 203 & 204 & 0 & 202 & 194 & 35 & 0 & 35 & 35 & .7 & 36 & $2 \pi$ & n & 35 & 35 \\
\hline FNOWMS FERRY-Z & 0 & 198 & 194 & 0 & 205 & 199 & 0 & 207 & 196 & $\Pi$ & 0 & 35 & 3 & n & 36 & 35 & 0 & 36 & 39 & 0 \\
\hline ROWHE FERAY - 3 & 0 & 194 & 197 & 0 & 200 & 203 & ס & 200 & $2 m$ & 0 & 0 & 36 & 35 & 0 & 35 & $3 x$ & 0 & 36 & 35 & $n$ \\
\hline RUNSW ICK-1 & 158 & 160 & 0 & 162 & 165 & 0 & 165 & 166 & 0 & 263 & 30 & 30 & 0 & 10 & 31 & 0 & 31 & 31 & [ & 30 \\
\hline RUNSh $1[K-Z$ & J & 159 & 162 & 0 & 165 & 166 & D & 166 & 163 & $\theta$ & 0 & 30 & 30 & 0 & 3] & 31 & 0 & 31 & 30 & $n$ \\
\hline rRUN-1 & 5) & 50 & 51 & 52 & $\$ 2$ & 53 & 53 & 53 & 52 & 52 & 22 & 21 & 22 & 22 & 22 & 22 & 22 & 22 & 22 & 22 \\
\hline RQW-? & 51 & 51 & 51 & 52 & 53 & 53 & $\$ 3$ & 53 & 52 & 52 & 22 & 22 & 22 & 22 & 22 & 22 & 23 & 22 & 22 & 22 \\
\hline $\operatorname{ALL} A \operatorname{An} \ln ^{\prime} Y-1$ & 7 & 79 & D & 90 & 82 & 0 & 82 & 82 & 0 & 81 & 33 & 33 & 0 & 34 & 35 & 0 & 35 & 35 & D & 34 \\
\hline CALVENT CLIFFS-1 & 65 & 65 & 0 & 85 & 67 & n & ह) & 67 & 0 & 68 & 25 & 25 & 0 & 25 & 26 & $\pi$ & 26 & 76 & $n$ & 26 \\
\hline CALVEPT CLIFES-Z & 65 & 0 & 65 & 66 & $n$ & 67 & 67 & 0 & 67 & 65 & 25 & 0 & 25 & 26 & 3 & 26 & 26 & 0 & $2 \hbar$ & 25 \\
\hline ATAUEA-I & $5_{4}$ & 57 & 54 & 55 & 59 & 57 & s) & 0 & $\$ 7$ & 54 & 23 & 24 & 23 & 23 & 25 & 24 & $7 d$ & ก & 24 & 23 \\
\hline ATANHA-2 & 54 & 57 & 53 & 58 & 5) & 0 & 57 & ร月 & 55 & 59 & 23 & 24 & 22 & 25 & 24 & $n$ & 24 & 25 & 23 & 25 \\
\hline LIMTrIN-: & $\Pi$ & 200 & 204 & 0 & $20 R$ & 210 & 0 & 211 & $2 n 7$ & B & $n$ & 37 & 37 & n? & 30 & $3 R$ & $n$ & 39 & $3 R$ & $\Pi$ \\
\hline COMAMCHE PEAK-1 & ss & 57 & $\$$ & $\leq$ & 59 & fin & Fo & 60 & 59 & 58 & 23 & 23 & 23 & 23 & 20 & 71 & 94 & 74 & 24 & 23 \\
\hline OMANCHE DEAK $=2$ & 59 & S4 & 58 & 59 & 59 & 60 & 60 & 59 & 59 & 50 & 74 & $? 3$ & 73 & 24 & 94 & $>s$ & 94 & 24 & 34 & ?ִ \\
\hline COHANET TICUT YAHKEE & 0 & 43 & 50 & 49 & ר? & 49 & 49 & s9 & 0 & $4 R$ & n & 17 & ח & 20 & n & $2 n$ & $2 n$ & 20 & 1 & $m$ \\
\hline LJOPEN & 103 & 102 & 102 & 102 & 106 & 105 & 108 & jins & 102 & $\ln 2$ & 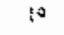 & 19 & 18 & 19 & 29 & 19 & 19 & la & 19 & 14 \\
\hline RSTHE RIVER-3 & 57 & 57 & ! & 58 & 59 & 0 & 59 & 59 & $?$ & SR & $2 \hbar$ & 26 & 0 & 27 & 27 & $n$ & 27 & 27 & ? & $2 ?$ \\
\hline c criok-j & $t^{2}$ & ] & 7 & 72 & 9 & 74 & 15 & ?: & TA & 13 & 37 & $n$ & 33 & 33 & $n$ & 34 & 34 & $\therefore$ & 34 & 73 \\
\hline$\therefore \quad \pi \cdot u k-2$ & t & i) & : & bI & $\mathrm{c}$ & 32 & 82 & 0 & Q1 & ती & 31 & a & 32 & 33 & ה & 13 & 13 & 7 & 33 & 32 \\
\hline $34 \mathbb{L}[5-8 \in 5 \$\{-1$ & 0 & 56 & 45 & 0 & 44 & 45 & i7 & 45 & 44 & 7 & 0 & 25 & 21 & ก & 21 & 21 & ה & $2 !$ & 21 & ï \\
\hline D] RELU CANYON-l & Sह & $\$ 6$ & SH & 3 & Sִ9 & 59 & 59 & 60 & Sis & 0 & 27 & 25 & 23 & 0 & 27 & 27 & 27 & $2 *$ & $? 7$ & 9 \\
\hline 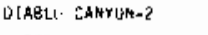 & 5? & 6 & 58 & 57 & 61 & 5 & kn & 0 & 57 & 57 & 26 & 0 & $2 ?$ & $2 \pi$ & $2 \rho$ & $2 ?$ & $2 P$ & \urcorner & $?$ & 95 \\
\hline JHESD: N-I & r. & 0 & C & 3 & 0 & 5 & 0 & ! & 0 & 0 & 9 & li: & $u$ & $n$ & 3 & 0 & ד. & i & v & $\therefore$ \\
\hline $50 \mathrm{OA}-2$ & 0 & $1 / 3$ & $! 76$ & 0 & 179 & lal & $n$ & 181 & $17 R$ & 0 & $n$ & 31 & 32 & ? & 37 & 33 & $n$ & נ3 & 32 & 9 \\
\hline ARE SDẼN-3 & 241 & 142 & 13 & 144 & 147 & $\Pi$ & IAB & $\{4\}$ & 0 & 145 & 26 & 26 & 0 & $2 ?$ & 27 & 0 & $2 ?$ & $2 ?$ & 0 & $? ?$ \\
\hline DUAT ARMOLD & ? & 106 & $20 B$ & a] & 110 & $1: 1$ & 0 & 111 & 109 & n & 0 & 19 & 27 & r. & $2 n$ & $r:$ & 0 & $? n$ & $2 r$ & $\curvearrowright$ \\
\hline ARLE $E^{Y}-1$ & 64 & 0 & 65 & 66 & 0 & 67 & 58 & 0 & 65 & 66 & 3n & 0 & $3 n$ & $3 n$ & i & 31 & 3l & 1 & $\because r$ & : \\
\hline$A R L E=-2$ & 64 & 85 & f. & 65 & S. & 7 & 5) & hi & $n$ & he & 29 & ?n & १ & 30 & $3 n$ & $n$ & 31 & 3 & " & 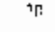 \\
\hline $2 M:-2$ & 0 & 259 & 257 & 0 & $26 \mathrm{~B}$ & 0 & 256 & 0 & 268 & $25, \rho$ & it & 87 & s? & గִ & 40 & 1 & 9.4 & : & 19 & $\therefore$ \\
\hline ITLPATRICK & 176 & 177 & 0 & $\operatorname{lB} \cap$ & 189 & $17 ?$ & 0 & JA7 & la्रे & $n$ & 37 & 37 & ก & 37 & 34 & 37 & 1 & 3 & 23 & . \\
\hline ORT $\quad$ ALLHOLIN-1 & 39 & r: & $4 n$ & $4 B$ & .] & 41 & sl & $n$ & 41 & 40 & 14 & 7 & 14 & i4 & $n$ & 15 & 15 & г. & 15 & 14 \\
\hline SANA & 26 & 25 & 26 & 26 & $2 \pi$ & 26 & 76 & $2 \pi$ & 26 & 25 & 9 & $a$ & $a$ & د & 0 & 9 & 0 & $\Rightarrow$ & 9 & g \\
\hline تRANQ GLAF-1 & 231 & 234 & a & 237 & 242 & 0 & 244 & 243 & $n$ & 239 & 11 & 41 & 7 & d? & 4.2 & r: & A & 43 & 1 & 17 \\
\hline SRAMO GULF-2 & 139 & $: 92$ & 191 & 193 & 199 & 199 & $2 \pi \mathbb{N}$ & $\mathrm{nnn}$ & 197 & : 조 & 1.3 & 35 & 35 & 35 & $3 F$ & 37 & l! & $7 !$ & th & it \\
\hline ARR I j -1 & 46 & 0 & 47 & 47 & 4.9 & $4 R$ & ה & 49 & Ath & 97 & 21 & B & 22 & 22 & 22 & 27 & $\hat{\imath}$ & 37 & 72 & 77 \\
\hline ARTS ILLLE-Al & 156 & 217 & 0 & 216 & 245 & 0 & 225 & 233 & ก & 231 & $? \mathrm{~A}$ & 4.3 & 0 & 39 & as & 7 & 11 & 47 & $n$ & $4 ?$ \\
\hline IRTS \& liLLE-A2 & U & (1) & 0 & 166 & 0 & 224 & 216 & ח & 244 & 222 & ? & 0 & It & 30 & ה & 43 & 39 & $\eta$ & $\Delta d$ & tn \\
\hline $\mathrm{TCH}_{-1}$ & 124 & 123 & 126 & 126 & $12 \mathrm{H}$ & 129 & 130 & เ Зก & $12 \mathrm{R}$ & 127 & 23 & $7 ?$ & 23 & 31 & 23 & 23 & $? 4$ & 70 & 23 & 22 \\
\hline
\end{tabular}


TABLE A.3. (contd)

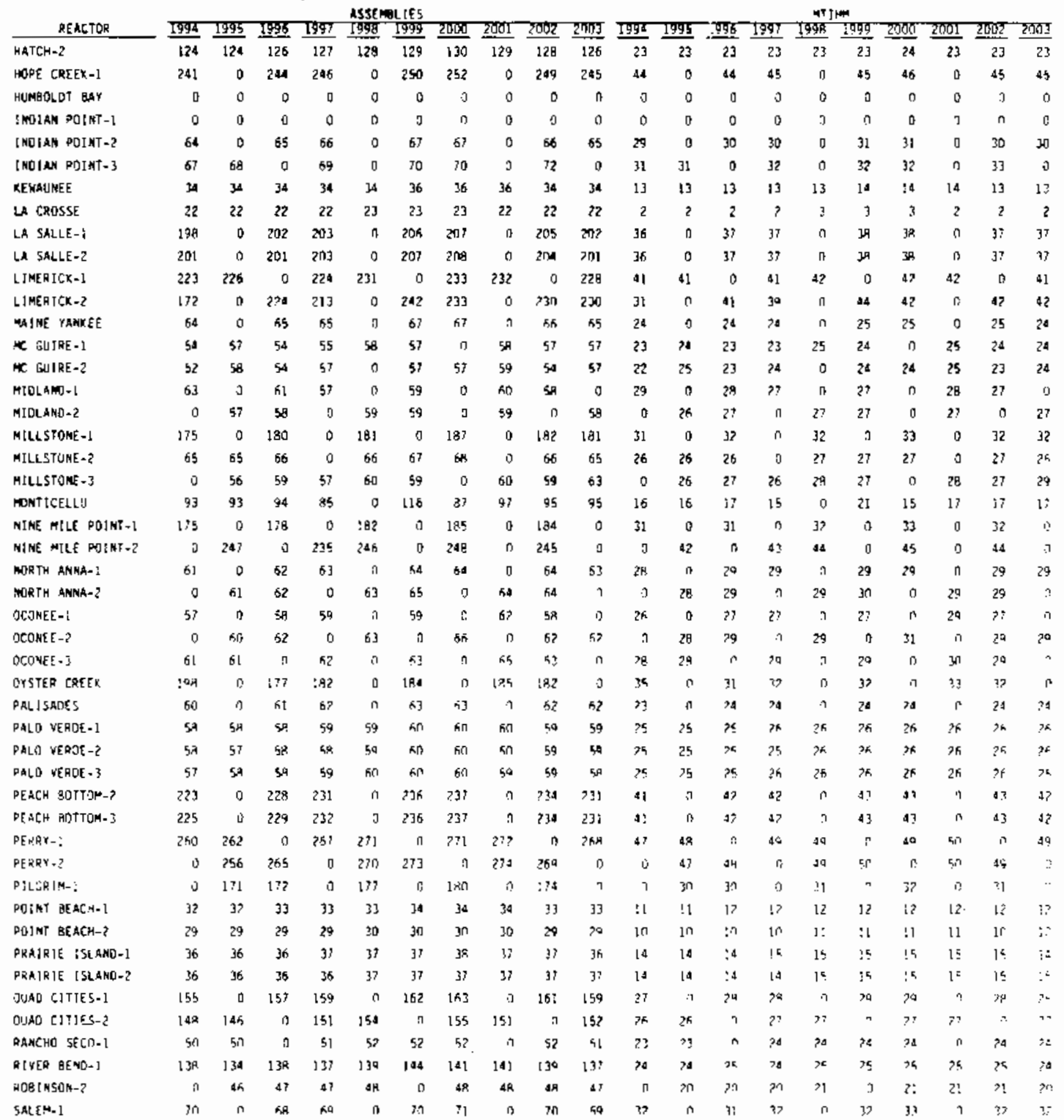


TABLE A.3. (contd)

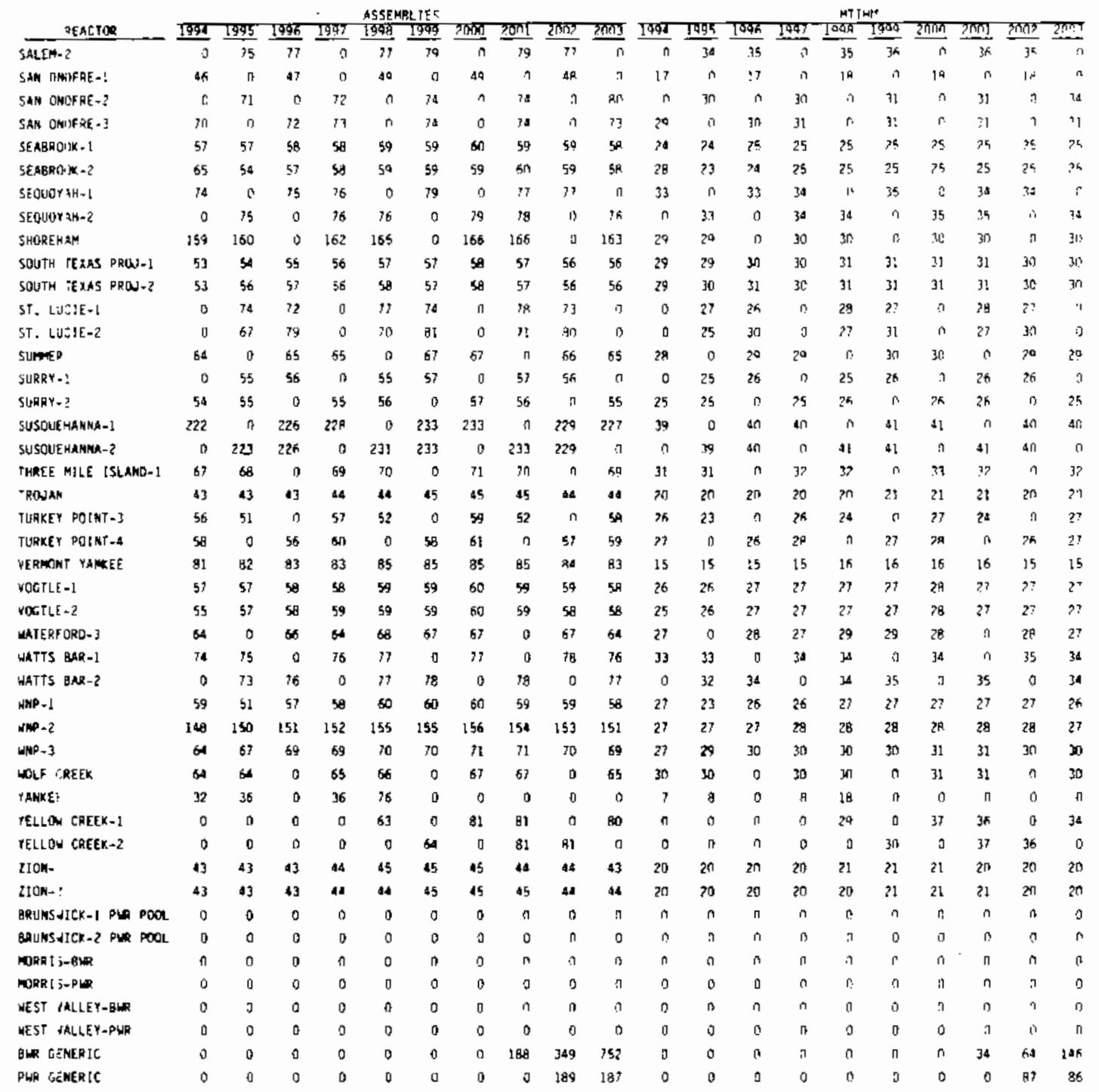


TABLE A.3. (contd)

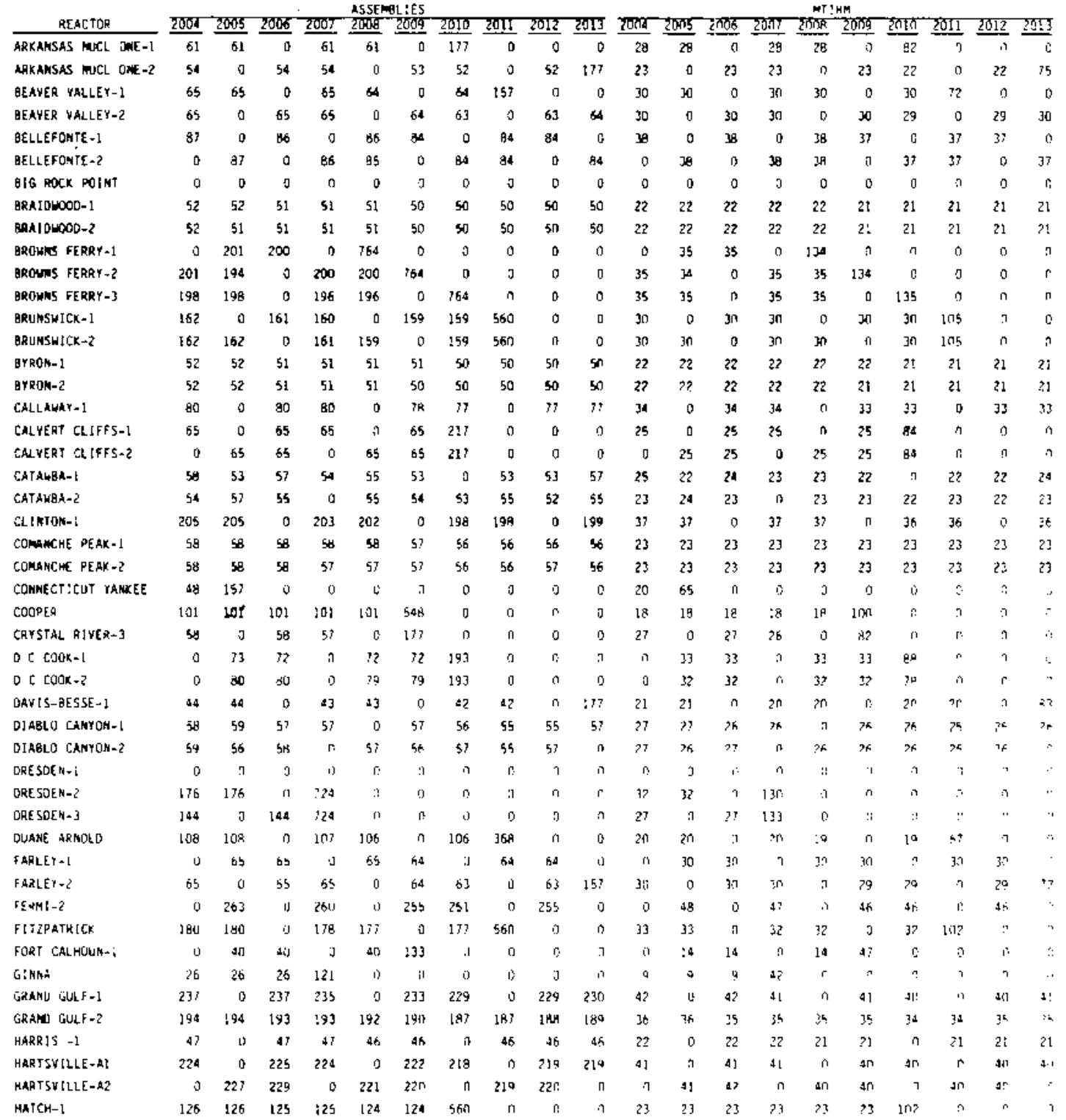


TABLE A.3. (contd)

\begin{tabular}{|c|c|c|c|c|c|c|c|c|c|c|c|c|c|c|c|c|c|c|c|c|}
\hline \multirow[b]{2}{*}{ QEAACTOR } & \multicolumn{10}{|c|}{ ASSEMSL IES } & \multicolumn{10}{|c|}{ जT! Hल } \\
\hline & 201 & 2005 & 2006 & 2007 & 20100 & 2009 & $2 \pi 10$ & 2011 & $\underline{2012}$ & $\underline{2013}$ & 2004 & 20095 & ?ती & 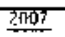 & 2तn & 20n9 & $20+15$ & 2011 & $27 \sqrt{2}$ & $3 \sqrt{313}$ \\
\hline HATCH-2 & 126 & 126 & 125 & 125 & 124 & 123 & 221 & 122 & 122 & 560 & 23 & 23 & 23 & 23 & 23 & 22 & 22 & $? 2$ & 72 & 102 \\
\hline HIDPE CAEEK-1 & 0 & 245 & 24] & [) & 242 & 278 & n & 236 & 233 & $\pi$ & $n$ & 25 & a s & 1 & 44 & 43 & $n$ & A & 43 & is \\
\hline NUMBOLDT BAY & J & 0 & J & 0 & נ & 0 & if & n & ח & n & 0 & n & $n$ & if & D & 7 & п & $n$ & : & 0 \\
\hline :.YDIAN DOINT-1 & 0 & i) & 0 & i) & $\eta$ & is & 0 & 1) & $n$ & 了 & 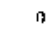 & 1 & n & ר? & ก & 0 & ה & 1 & n & 7 \\
\hline IMIIAM POINT-2 & 7 & 55 & 65 & 193 & 3 & 0 & ? & 1) & 0 & ) & $n$ & 30 & $3 n$ & घ9 & i) & 7 & o & $n$ & ه & 1 \\
\hline 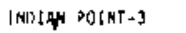 & 68 & 67 & 0 & 10 & $x_{6}$ & is & 193 & ) & $n$ & 7 & 31 & 31 & $\pi$ & 32 & in & i) & .95 & ה & $n$ & 0 \\
\hline X E UAUMEEE & 34 & 34 & 34 & 34 & 34 & 121 & $\mathrm{u}$ & 0 & 0 & 0 & 13 & \{\} & 13 & 13 & 13 & 46 & 3 & D & 0 & 3 \\
\hline LA CADSSE & 72 & s & 0 & 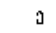 & 1) & ?] & n & o & 0 & $\jmath$ & 8 & 0 & 0 & ] & n & 0 & 0 & i) & n & i) \\
\hline LA SALLE-1 & 0 & 202 & 200 & 0 & 199 & 196 & 0 & 194 & 195 & D & 0 & 37 & 16 & 0 & 36 & 36 & ] & 35 & 36 & i \\
\hline LA SALLE-2 & 0 & 201 & 200 & $a$ & 199 & 195 & 0 & 194 & 195 & 0 & 0 & 37 & 36 & $D$ & 36 & 36 & i] & 35 & 36 & 7 \\
\hline LI YER]CK-1] & 226 & 0 & 226 & 224 & a & 222 & 219 & 0 & 219 & 220 & $\$ 1$ & $a$ & 41 & 41 & $D$ & 40 & so & 0 & 40 & 40 \\
\hline Li TERICK-2 & 0 & 224 & 225 & a & 224 & 220 & 0 & 219 & 220 & 0 & 0 & 41 & 41 & 0 & al & $4 / 7$ & 丁 & 40 & 10 & $\Pi$ \\
\hline TA:NE YAMKEE & 0 & 65 & of & 9 & 55 & 217 & 0 & 0 & 3 & $n$ & 0 & 24 & 24 & 0 & 24 & .11 & 0 & o & i) & 0 \\
\hline$\triangle$ GUIRE-1 & 54 & 58 & 54 & 54 & 55 & ง & 53 & 55 & 53 & 53 & 23 & 25 & 23 & 23 & 23 & 0 & 22 & 23 & 22 & 22 \\
\hline$X$ CEIRE-2 & 55 & 55 & 55 & D & 54 & 55 & 52 & 55 & 52 & 52 & 23 & 23 & 23 & $n$ & 23 & 23 & 22 & 23 & 22 & 22 \\
\hline MISLANO-1 & $\$$ & 0 & 48 & 57 & $n$ & 56 & 0 & 56 & 56 & $n$ & 27 & a & 27 & 27 & is & 26 & 0 & 26 & 26 & 0 \\
\hline DLAMO-2 & 58 & a & 58 & 57 & 1) & 57 & 56 & 0 & 56 & 0 & 21 & ?t & 27 & 27 & $n$ & 27 & 26 & ? & 26 & 0 \\
\hline CLSTONE-I & בם & lBo & D) & 581 & D & n & n & 0 & $n$ & $n$ & $\pi$ & 39 & $\mathrm{n}$ & 10? & $n$ & $n$ & ) & $n$ & $n$ & in \\
\hline LLSTONE-2 & 66 & $n$ & 65 & 64 & 65 & in & 55 & 217 & a & ก & 26 & 1 & $2 h$ & 26 & 26 & n & 26 & 87 & ] & D \\
\hline :LSTOME-3 & 49 & 52 & 59 & 57 & 57 & $n$ & 56 & 56 & 56 & 56 & 22 & 29 & 27 & 76 & $2 \pi$ & 0 & 25 & 26 & $2 \pi$ & 26 \\
\hline NTICELLO & 95 & 74 & 93 & 7 & งคd & n & n & ) & ก & i) & 17 & 19 & 16 & $\eta$ & Af & 0 & $n$ & 0 & 3 & ก \\
\hline YIYE ज!LE POLभT-1 & $! 90$ & 0 & 532 & $n$ & 7 & 9 & n & $n$ & $B$ & n & 32 & $n$ & צA & $n$ & 1 & n & 1 & 1) & 9 & B \\
\hline YTUE MLLE PDTHT-Z & 241 & $24]$ & 0 & 239 & 0 & 236 & ה & 232 & 233 & a & 43 & 43 & 1) & 43 & a & $\Delta$ & D & $1 ?$ & 17 & 7 \\
\hline YUBRTH AHNA-1 & a & 62 & $6 !$ & 1 & B2 & 60 & i & 50 & 157 & ה & ] & 29 & 2马 & $n$ & 29 & 개 & 0 & $? 9$ & $? 2$ & 7 \\
\hline NUPTH ANMA-2 & $n_{2}$ & 52 & 0 & $\$ 1$ & 52 & ] & $\dot{S_{0}}$ & 50 & 157 & 0 & 29 & 29 & 0 & 28 & 29 & 0 & 29 & 28 & 12 & i) \\
\hline TCCNEE - 1 & 58 & 1) & 58 & 58 & .1 & $17)$ & $\Rightarrow$ & 0 & 0 & ד & 27 & 0 & 27 & $2 ?$ & ง & 32 & n & $\eta$ & .9 & 0 \\
\hline J J:IKEE - ? & ; & 82 & 0 & 52 & $i 7$ & $\mathrm{~J}$ & i) & ] & 1) & J & (1) & 29 & 9 & 29 & A2 & 2 & : & 1) & 1 & $i^{1}$ \\
\hline IT,.NAEE - $]$ & 53 & 61 & 0 & $\delta 1$ &.$j$ & 177 & a & 0 & i) & 0 & 29 & 28 & J & $2 \mathrm{~B}$ & ד & $\mathrm{H} 2$ & $n$ & 7 & $n$ & B) \\
\hline JYSTEK CAEEX & $i z 2$ & 560 & c & 1 & i) & ] & 0 & i) & 0 & J & 32 & 99 & 0 & J & 0 & 1) & a & 1) & 1 & ) \\
\hline I I JADEE 5 & 1) & fil & 61 & $\Rightarrow$ & 204 & 0 & .] & .) & i: & 1) & 3 & 24 & 24 & $\eta$ & 39 & B & I] & $\pi$ & $n$ & 9 \\
\hline DALIJ YERDE - 1 & ริ & 58 & मे & $5 B$ & 纳 & 57 & 56 & 56 & 57 & y) & 75 & 25 & 35 & 25 & 25 & 25 & 24 & 24 & 25 & 25 \\
\hline DHIO VERDE - Z & 50 & 59 & sà & 58 & 58 & 58 & Sf & 56 & 57 & 57 & 25 & 26 & 25 & 25 & 25 & 25 & 24 & 24 & 25 & 25 \\
\hline 2AL U YERDE-3 & y & 涑 & $5 \mathrm{~A}$ & $S \mathrm{~B}$ & 58 & 56 & 56 & 56 & 57 & 57 & 26 & 25 & $? 5$ & 25 & 25 & 24 & 24 & 20 & 25 & 25 \\
\hline DE-CH HOITOM- & $\therefore$ & 230 & 229 & $n$ & 229 & .54 & .9 & i) & ก & i) & חי & 42 & $1 ?$ & $n$ & 12 & 139 & $n$ & .1 & i & $\therefore$ \\
\hline$p[\therefore: . H \quad$ ЗLTTUM-3 & 了 & 230 & 229 & 1) & 223 & 164 & 1) & 1 & 1 & 3 & 0 & 12 & 1? & $J$ & む? & 139 & 了 & 0 & 7 & I \\
\hline sfi:kY-l & 254 & 1 & 265 & 26.4 & 1 & 251 & 257 & 11 & 257 & 268 & 49 & 0 & 19 & $A R$ & 1 & 4.9 & 47 & $\pi$ & 47 & $1 ?$ \\
\hline$=[: R \times-2$ & $? 54$ & $2 ! 2$ & 1 & 363 & 2501 & 1 & 757 & 757 & $n$ & 550 & 4) & 15 & 9 & 48 & 47 & $n$ & 17 & 47 & . & 4) \\
\hline P:I GR IM-1 & $! 34$ & !? & i] & 172 & 7 & $58 n$ & 7 & "י & 7 & $n$ & 31 & 11 & 1 & 波 & 1 & 103 & 7 & i & 9 & 1) \\
\hline$M$ WF $3 E A C H=1$ & 33 & J] & $3]$ & $3]$ & $: 21$ & 0 & $n$ & ו. & 0 & 1 & $! ?$ & 12 & $\vdots 2$ & 12 & 43 & $n$ & .1 & $n$ & f, & 7 \\
\hline $2 \pi N$ NEACH-? & $? 7$ & 29 & 29 & 29 & 29 & $1 ? .1$ & ה & $n$ & 1 & i) & in & 10 & $\therefore$ & In & In & 47 & $n$ & $\therefore$ & 7 & ה \\
\hline DOLIE :SLAND-i & 36 & 26 & 36 & 36 & J6 & 121 & $n$ & . & 13 & .1 & $: 1$ & 14 & is & 14 & $: 4$ & 49 & : & 11 & 8 & 1 \\
\hline SR:JRIE ISLAHO-Z & 16 & 36 & 36 & 26 & 36 & 121 & .1 & 1) & 1 & 0) & 14 & ls & is & ls & $: 4$ & دA & n & n & ר. & i] \\
\hline AIAD CITTES-l & : & : 58 & $15 \beta$ & 11 & $? ? 4$ & in & 13 & $n$ & 1) & ] & $n$ & ${ }_{A B}$ & 2h & 1 & :28 & $n$ & \urcorner & $n$ & r: & 1 \\
\hline ;J:D CITIES=2 & 151 & 1 & 148 & 125 & :] & $\mathrm{n}$ & .7 & i) & 1 & 13 & 27 & $n$ & $2 \pi$ & $12 \mathrm{R}$ & 0 & .1 & $n$ & $n$ & .7 & 0 \\
\hline ২AMCHU SECO-1 & 31 & 51 & $J$ & 51 & 51 & 177 & 0 & J & 3 & 1) & 24 & 24 & in & 24 & 24 & 32 & 3 & 0 & 1) & 0 \\
\hline स['ER AEND-1 & :44] & 137 & 136 & $: 36$ & 138 & 134 & $! 32$ & 132 & $: 36$ & 133 & 24 & 24 & 24 & 24 & 24 & 20 & $2]$ & 23 & 24 & 24 \\
\hline AUo 6 HSUN-2 & i) & 37 & 47 & 157 & 0 & 0 & 0 & $\cdot$ & 1) & $\mathrm{j}$ & !) & 20 & 20 & 58 & i & i) & 0 & " & i) & ง \\
\hline VAL ミH-! & .j & 69 & 56 & 9 & 58 & 193 & 0 & 0 & ה & 0 & 0 & 32 & 31 & $n$ & 31 & 89 & D & c & 0 & 0 \\
\hline
\end{tabular}


TABLE A.3. (contd)

\begin{tabular}{|c|c|c|c|c|c|c|c|c|c|c|c|c|c|c|c|c|c|c|c|c|}
\hline & & & & & A5SEN & & & & & & & & & & & & & & & \\
\hline REACTOR & tod & 2005 & 3006 & 2007 & 2006 & 20019 & 2010 & 2011 & $2 \sqrt{212}$ & 2019 & 2000s & $2005 !^{-}$ & 2006 & 2007 & 200त्व & 2009 & खणता & खा1 & 2012 & गुI \\
\hline SALEM-2 & $n$ & 77 & 0 & 15 & 75 & 0 & 193 & 0 & ป & 0 & 35 & $3 \pm$ & 0 & 34 & 34 & 0 & 39 & 0 & $\overline{0}$ & 0 \\
\hline SAM UNOFRE-I & 49 & 157 & 0 & 0 & 0 & 0 & م & $?$ & 0 & 0 & 18 & se & 0 & 0 & 0 & 0 & 0 & 0 & is & 门 \\
\hline SAN ANOFRE-2 & 99 & 0 & 39 & 0 & 11 & 0 & to & ) & 70 & 0 & 25 & " & 33 & 0 & 30 & 0 & 29 & 3 & 29 & a \\
\hline SAN UMOFAE-3 & 0 & 72 & 0 & 72 & 0 & 11 & 0 & 10 & $D$ & 70 & 0 & JC & 0 & ]ง & 0 & 10 & 0 & 29 & J & 29 \\
\hline SEABRDOC -1 & 5 & 50 & 58 & 57 & 57 & 56 & So & $\$$ & 56 & 56 & 25 & 26 & 25 & 24 & 24 & 24 & 24 & 24 & 24 & 24 \\
\hline SEABROOK-2 & 58 & 98 & 幽 & 57 & 57 & $5 t$ & so & so & 56 & 56 & 25 & $2 \xi$ & 25 & 24 & 24 & 20 & 24 & 24 & 24 & 24 \\
\hline SEQNOYAAH - I & 76 & 36 & 0 & 75 & 75 & 0 & 75 & 0 & 193 & 0 & $\mathbf{4}$ & $\mathbf{4}$ & 0 & 33 & 33 & 0 & 33 & 0 & 86 & 0 \\
\hline SEQUOYAH+2 & 76 & 0 & 75 & 0 & 15 & 74 & 0 & 193 & 0 & 0 & 34 & $c$ & 33 & 0 & 33 & 13 & 0 & B6 & a & 0 \\
\hline SHOAEHAM & 162 & 0 & 162 & 161 & 0 & 159 & 156 & o & 156 & 157 & 30 & c & 10 & 30 & 0 & $x$ & $\rtimes$ & 0 & $\mathbf{q}$ & 29 \\
\hline SQUTH TEXAS PROU-1 & 56 & st & 56 & 55 & 55 & 54 & sis & 54 & s4 & 54 & 30 & $3 x$ & 30 & 30 & 30 & 20 & 29 & $x$ & 29 & 29 \\
\hline SOUTH TEXAS PRDAS-2 & 56 & 56 & 56 & 55 & 55 & 54 & 44 & $s$ & 54 & 54 & 10 & 36 & 10 & 30 & 30 & 3 & 29 & 29 & 29 & 29 \\
\hline ST. LUCIE- & 36 & 12 & 0 & 75 & 70 & 1) & 30 & 217 & $n$ & 0 & $2 B$ & 26 & ) & 27 & $2 \pi$ & 7) & 26 & 39 & .7 & 0 \\
\hline 57. LUCIE-2 & 59 & $\mathrm{~g}$ & 0 & 49 & ?g & o & fos & $n$ & a & 6) & 26 & $x$ & 0 & 26 & 30 & D & 25 & 29 & 0 & 25 \\
\hline SUIMER & 0 & 65 & 55 & 0 & $\omega$ & 53 & 0 & 63 & 63 & 0 & 0 & 29 & $x 9$ & a & $2 \mathbf{R}$ & 28 & 0 & $2 \beta$ & 28 & 7 \\
\hline 5.JRRY Y - & 55 & 56 & 0 & 55 & 55 & a & 157 & 0 & g & 0 & 25 & $2 t$ & 0 & 25 & 25 & 0 & 12 & 0 & 0 & i) \\
\hline SURAT-2 & $\mathbf{4}$ & a & 55 & $\$ \$$ & 0 & 157 & 0 & 0 & 0 & 0 & 26 & $c$ & 25 & 25 & 0 & 12 & 3 & 0 & 0 & a \\
\hline SUSONEHAHIM +1 & 0 & 227 & 225 & g & 223 & 219 & 0 & 219 & 220 & 0 & 0 & sc. & $\omega_{0}$ & a & 39 & 39 & 0 & 39 & 39 & 0 \\
\hline SUSQUEMAAML-2 & 226 & 226 & o & 225 & 223 & 0 & 218 & 219 & ) & 220 & 40 & $\propto$ & o & 40 & 39 & J & 30 & 39 & 5 & 39 \\
\hline PNREE MILE ISL AND-1 & 69 & 0 & 58 & $\Leftrightarrow 8$ & 0 & 17 & 0 & g & D & 0 & 32 & $\mathrm{c}$ & 31 & 31 & 0 & 82 & 0 & 0 & 0 & 0 \\
\hline TRUNAN & 44 & 44 & 43 & 43 & 43 & 42 & 42 & 42 & 193 & 0 & 20 & $2 c$ & 20 & 20 & 20 & 19 & 19 & 19 & 89 & 0 \\
\hline TURKEY POINT-3 & 51 & 0 & 59 & s & 0 & 157 & 0 & 0 & 0 & 0 & 23 & c & 21 & 27 & ง & i2 & 0 & i) & 0 & o \\
\hline TURKEYY POLNT-4 & ป & 57 & 59 & 0 & [5] & 0 & 0 & 0 & 0 & 0 & $n$ & $2 \epsilon$ & 3 & דם & 72 & D) & 了) & 7 & 3 & 门 \\
\hline WERMAMT YANKEE & 83 & B3 & 83 & 83 & 368 & a & 0 & id & 0 & 0 & 15 & 15 & 15 & $: 5$ & 87 & 0 & 3 & 0 & 1) & 3 \\
\hline WOGTLE-I & s & 50 & 58 & 57 & 57 & 56 & 58 & S6 & $\$$ & 56 & 21 & 27 & 27 & 26 & 26 & 26 & 26 & 26 & 26 & 26 \\
\hline MGLE-2 & 58 & 50 & 59 & 57 & 57 & 56 & $\$ 6$ & 56 & 56 & 56 & 27 & 27 & 27 & 26 & 26 & 26 & 25 & 26 & 26 & 26 \\
\hline WATERFORO-3 & 5) & 54 & 65 & 3 & 55 & 62 & 65 & 52 & 63 & $\mathrm{~B}$ & 28 & 27 & 29 & $\eta$ & 28 & 26 & 28 & 26 & 27 & ง \\
\hline WATTS RAR-1 & 0 & 16 & 15 & 0 & 75 & is & 0 & 13 & ) & 74 & $n$ & 34 & 33 & $n$ & 33 & 33 & 0 & 32 & 0 & 33 \\
\hline NATTS BAF-2 & 15 & 0 & 76 & 15 & 0 & id & : 3 & n & 73 & וי & 33 & 6 & 34 & 33 & 0 & 33 & 32 & $n$ & 32 & ה \\
\hline :MP - 1 & 58 & 50 & 58 & 57 & 57 & 56 & 58 & 56 & $s_{f}$ & 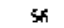 & 26 & $2 t$ & $2 \pi$ & 26 & 26 & 26 & 26 & 26 & 26 & 26 \\
\hline $\ln _{1} P \cdot \theta-2$ & {$[5]$} & 151 & 150 & !50 & 149 & 146 & 146 & 146 & 147 & 146 & $\eta$ & $n$ & $? 7$ & 27 & $\eta$ & 77 & 21 & $\eta ?$ & 27 & 27 \\
\hline AP-] & و & 59 & $\infty 8$ & $6 \mathrm{~B}$ & $\Leftrightarrow$ & 6) & КБ & Sא & 56 & 67 & 30 & 35 & 29 & 29 & 29 & $x$ & 28 & $2 B$ & 28 & $x$ \\
\hline WILF CREEK & אם & 0 & 55 & fi. & i) & 54 & 6] & D & 63 & 6] & 30 & $i$ & 30 & 30 & 0 & 30 & 99 & in & 29 & 29 \\
\hline WMKEE & נ. & $n$ & 1) & 0 & ? & 1) & 3 & . & م & $\pi$ & 7 & $:$ & 0 & .1 & 0 & D & ه & $\pi$ & i) & n \\
\hline "EL:OH CREEK-1 & 81 & 0 & SO & คด & 0 & 湜 & 77 & $n$ & $n$ & $n$ & s & 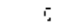 & 74 & 34 & D & 33 & 33 & $n$ & 13 & 33 \\
\hline CELLUWW CREEK = ? & 30 & $\$ 1$ & u & मn & 19 & i) & $\eta$ & $n$ & $n$ & 77 & 34 & 34 & 0 & 34 & 33 & 1) & 33 & 33 & 1 & 3 \\
\hline $2: 0 N-1$ & 24 & 43 & 43 & 43 & 43 & 193 & $n$ & $n$ & .1 & ה & 20 & $2 r$ & $2 n$ & $?$ & 20 & pg & 0 & o & $n$ & 0 \\
\hline Z1UN-2 & 43 & 4d & 13 & \pm 3 & 43 & 193 & ס & ורי & 0 & J & 20 & $2 t$ & 20 & 20 & 20 & HA & 0 & a & 3 & o) \\
\hline HRUNSWICK-L PU POOL & 0 & 0 & 1) & J & 1 & 1) & ון & 0 & g & וי וי & $n$ & i &. & 0 & ה & $\mathrm{n}$ & 1 & $\mathrm{n}$ & $n$ & 0 \\
\hline dRLINSNICK-2 PUR POOL & ل & 1] & u & u & ] & 1] & 0 & ני & 0 & J & $\therefore$ & . & 0 & J & 0 & 0 & 1) & J & J & 0 \\
\hline MLLRR 15-日LR & 0 & 4 & 'p & 0 & a & n & 0 & 1) & 1 & וי & i & $\therefore$ & 0 & 1) & 0 & n & 9 & 1 & ) & 0 \\
\hline 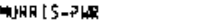 & J & u & 1) & 0 & j] & 0 & 0 & J & 1) & 1 & 1] & : & 0 & .1 & : & 0 & 0 & J & 3 & a \\
\hline TEST VALLEY-BWA & 0 & 3 & 0 &. & 0 & ה & 0 & 1) & 1 & 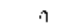 & $n$ & i & j] & ה & 1) & i) & $a$ & n & ח & n \\
\hline .EST VAZLEY_PLA & a & 0 & a & 0 & נ] & 1) & 0) & 0 & 1) & ? & D) & $\varepsilon$ & $\mathrm{u}$ & 1 & 0 & 0 & 0 & 9 & 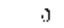 & 11 \\
\hline aLR TiENERIC & 927 & $: 226$ & 1221 & 2353 & 2220 & 3113 & 3765 & 5381 & 4280 & 49/म & 175 & 236 & 229 & $\$ 44$ & 408 & SR2 & m3 & 1017 & 794 & 911 \\
\hline PWR UENERTE & $\$ 26$ & $/ 42$ & 122 & 752 & 1467 & 1431 & 2009 & 2361 & 3048 & $8+3$ & 197 & 337 & 328 & 333 & 550 & 635 & 300 & เดรค & 1356 & 1293 \\
\hline
\end{tabular}


TABLE A.3. (contd)

\begin{tabular}{|c|c|c|c|c|c|c|c|c|c|c|c|c|c|c|}
\hline \multirow{2}{*}{ REACTCP } & \multirow{2}{*}{\multicolumn{7}{|c|}{ 255F MLIES }} & \multicolumn{7}{|c|}{ MT I I MM } \\
\hline & & & & & 2028 & 3019 & 2020 & 2014 & 2015 & 2016 & 2177 & खा? & $2 \pi 19$ & $2 \sqrt{220}$ \\
\hline ARKANSAS WUCL OME-1 & 0 & 0 & 0 & 0 & a & 0 & 0 & o & 0 & గ & 0 & ) & 0 & 0 \\
\hline ARKAMSAS MUCL ONE-2 & 0 & 0 & 0 & 0 & ก & n & 0 & a & $\pi$ & $n$ & $\Pi$ & מ & 0 & n \\
\hline EEAYER VNLLEY - I & 0 & 0 & i) & 0 & 0 & 0 & 0 & 0 & 0 & 0 & 0 & 0 & 0 & D \\
\hline BEAYER YALLEY $=2$ & 0 & so & 6 & 0 & 6 & המ & 0 & a & 30 & 30 & a & 30 & 30 & 0 \\
\hline GELLEFOMTE-1 & Q & 84 & 0 & BS & D & 85 & as & 37 & 37 & 0 & 37 & 0 & 37 & 37 \\
\hline SELLEFOMTE - 2 & ga & 0 & 94 & 0 & 85 & 85 & 0 & 37 & 0 & 37 & 0 & 18 & 38 & 0 \\
\hline BIG AOCK POINT & 0 & 0 & a & 0 & 0 & 0 & 0 & 0 & $t$ & 0 & 0 & 0 & 0 & 0 \\
\hline BRAIOLO00-1 & 50 & 50 & 50 & 50 & 51 & 53 & \$1 & 21 & 21 & 21 & 21 & 22 & 22 & 22 \\
\hline GRASOLOOO-2 & 50 & So & 50 & 51 & 51 & 51 & 51 & 21 & 21 & 21 & 22 & 22 & 22 & 22 \\
\hline EROWIS FERAY-1 & a & 0 & 0 & 0 & 0 & 0 & 0 & D & 0 & D & 0 & 0 & 0 & 0 \\
\hline SADHNS FERKY-2 & 0 & 0 & 0 & $\mathfrak{a}$ & 0 & o & 0 & 0 & 0 & 0 & 0 & 0 & 0 & 0 \\
\hline BROWTS FERRY-3 & 0 & 0 & 0 & 0 & 0 & 0 & 0 & 0 & i & 0 & 0 & 0 & $n$ & 0 \\
\hline BAUHSWICK-1 & a & 0 & 0 & 0 & 0 & 0 & 0 & 0 & 0 & 0 & 0 & o & 0 & 0 \\
\hline BRLMSWICX-2 & 0 & a] & 0 & 0 & 0 & 0 & 0 & 0 & 0 & 0 & 0 & 0 & D & 0 \\
\hline BYAOM-1 & so & so & so & so & 51 & 51 & 51 & 21 & 21 & 21 & $2 \mathrm{I}$ & 22 & 22 & 22 \\
\hline BYRON-2 & 50 & 50 & 50 & So) & 51 & 51 & 51 & 21 & 21 & 21 & 21 & 22 & 22 & 22 \\
\hline CALLAHAY $=1$ & a & $n$ & 78 & 0 & 78 & 78 & 0 & 0 & 33 & 33 & D & 33 & 33 & 0 \\
\hline CALVERT CLIFFS-I & 0 & $\pi$ & 0 & 0 & 0 & n & 0 & 0 & $n$ & n & n & $n$ & $n$ & 6) \\
\hline CALYERT CLLFF5-2 & 0 & 0 & 0 & 0 & 0 & 0 & 0 & 0 & 0 & 0 & $n$ & 0 & 0 & 0 \\
\hline [ATAMEA-1 & 52 & 53 & 55 & 50 & 54 & $\Pi$ & 54 & 22 & 22 & 23 & 23 & 23 & 0 & 23 \\
\hline CATAHBA-Z & 52 & 55 & 0 & 54 & 55 & 50 & 54 & 22 & 23 & 0 & 23 & 23 & 23 & 23 \\
\hline CLIMTON-1 & 198 & 0 & 200 & 201 & 0 & 201 & 202 & 36 & ी & 37 & 37 & 0 & 37 & 37 \\
\hline COMAHCHE PEAK=1 & 56 & 56 & 57 & 57 & 57 & 57 & 57 & 23 & 23 & 23 & 23 & 23 & 23 & 23 \\
\hline COMANCHE PE,AK-? & 56 & $5 ?$ & 57 & 57 & 57 & 57 & 57 & 23 & 23 & 23 & 23 & 23 & 23 & 23 \\
\hline CQNAECTICUT YAMEE & 0 & 0 & 0 & 0 & 0 & 0 & 0 & v & 0 & 0 & D & 0 & a & 0 \\
\hline COOPER & 0 & $\Pi$ & 0 & 0 & 0 & 0 & 0 & 0 & 0 & $n$ & 0 & c & $n$ & n \\
\hline CRYSTAL RLVER-3 & 0 & 0 & o & 0 & 0 & 0 & 0 & 0 & 0 & 0 & 0 & 0 & 0 & r \\
\hline D $[\cos x-1$ & 0 & $\pi$ & 0 & 0 & $\mathfrak{n}$ & 0 & $n$ & 0 & 0 & 0 & 0 & 0 & n & 0 \\
\hline D C c cook-2 & 0 & 0 & a & 0 & 0 & 0 & 0 & 0 & 0 & 0 & 0 & 0 & 0 & $n$ \\
\hline OAV T5-8ESSE-1 & 0 & 5 & 0 & 0 & 0 & 0 & 0 & 0 & $B$ & n & G & 0 & ים & $r$ \\
\hline DJABLLO CANYON-I & ] & 56 & 57 & 56 & 56 & 57 & 0 & 0 & 26 & 26 & 26 & 26 & $2 \hbar$ & 0 \\
\hline OIABLD CANYON-? & 56 & 55 & 58 & 55 & 57 & 0 & 57 & 26 & 25 & 27 & 25 & 26 & $n$ & $2 F$ \\
\hline RRESOEN-1 & 1 & 0 & n) & 0 & 0 & 0 & a & 0 & 0 & 0 & 0 & $n$ & 0 & ? \\
\hline DAE 5DE $\mathrm{N}=2$ & 0 & " & $n$ & 0 & n & n & $n$ & $\mathrm{o}$ & $r$ & $n$ & $n$ & 1 & $n$ & $r_{i}$ \\
\hline DRE $50 \mathrm{EN}-3$ & 0 & 0 & 0 & 0 & 0 & 0 & 0 & n & $n$ & 1 & n & 0 & 0 & $n$ \\
\hline DUAMC ARMOLD & 0 & n & $\pi$ & D & ๆ & $n$ & $n$ & $n$ & n & n & n & $n$ & $\pi$ & $\Gamma$ \\
\hline FARL\{Y-l & 157 & 0 & a & 0 & 0 & 0 & .7 & 12 & 0 & $\pi$ & n & n & f & $n$ \\
\hline SARLEY - 2 & 0 & $n$ & n & 0 & $n$ & n & 0 & i & $n$ & n & r & ก & 7 & $n$ \\
\hline FERMI -2 & 253 & 253 & o & 250 & $n$ & 260 & $\pi$ & th & 96 & $\pi$ & 15 & 7 & 47 & $n$ \\
\hline FTTZPATRJEX & J & 0 & ก & 0 & 7 & 0 & $n$ & n & ก & n & $\pi$ & $n$ & הם & 1 \\
\hline FORT CALHOUN-; & 0 & 0 & 0 & 0 & 0 & 0 & 0 & 0 & 0 & 0 & 0 & 9 & 0 & D) \\
\hline GINMA & $a$ & 0 & 1 & 0 & 0 & $n$ & n & 0 & a & $n$ & n & n & . & $\pi$ \\
\hline LRAMD [aلjLF $=1$ & 0 & 230 & 231 & 0 & 232 & 233 & 0 & a & 41 & 41 & 0 & al & 4: & $\eta$ \\
\hline GAANO GULF-? & 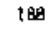 & 188 & 189 & 190 & 190 & 191 & 191 & 35 & 35 & 35 & 35 & 35 & 35 & 35 \\
\hline HAARIS -1 & 46 & $\square$ & 46 & 46 & 46 & 46 & 0 & 21 & 2 & 21 & 21 & 2: & 21 & a \\
\hline HARTSYILLE-AJ & 0 & 220 & 221 & 0 & 222 & 222 & 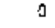 & 0 & an & 40 & 0 & 40 & 40 & 0 \\
\hline MARTSYILLE-A2 & 219 & 220 & 0 & 221 & $z 22$ & 0 & 223 & 40 & 40 & 0 & 40 & An & n & 41 \\
\hline HATCH-1 & D & 0 & 0 & 0 & 0 & 0 & 0 & 0 & $n$ & $n$ & 0 & 0 & 0 & 0 \\
\hline
\end{tabular}


TABLE A.3. (contd)

\begin{tabular}{|c|c|c|c|c|c|c|c|c|c|c|c|c|c|c|}
\hline \multirow[b]{2}{*}{ REACTOM } & \multicolumn{7}{|c|}{ ISSENGL IES } & \multicolumn{7}{|c|}{$T \Gamma$ L } \\
\hline & 2019 & 2015 & 2016 & $20 ! 7$ & 2018 & 2019 & 2020 & 20IT & 2015 & $\underline{2 \pi} \cdot \underline{6}$ & 2017 & 2912 & एगज & 2020 \\
\hline HNTCM-2 & 00 & 0 & 0 & 0 & a & 0 & 0 & 0 & 0 & 0 & 3 & 0 & b & \\
\hline HOPE CREEK-1 & 237 & 237 & 0 & 239 & 240 & 0 & 240 & 43 & 4] & g & 43 & $4 \mathbb{4}$ & 0 & 44 \\
\hline HUMOLLOT BaY & 1 & 0 & a & a & n & 0 & 0 & 0 & 0 & ) & i] & i) & 0 & 0 \\
\hline INOLN POINT-1 & 0 & 0 & 0 & 0 & 0 & a & 0 & D & a & Ј & D & 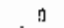 & J & $\vec{u}$ \\
\hline JMDIAN POLTR-Z & 0 & 0 & 0 & 0 & 0 & 0 & 0 & 0 & ס & 0 & a & 0 & 0 & 0 \\
\hline f & 0 & 0 & 0 & 0 & 0 & 0 & 0 & D & n & $\pi$ & ) & 0 & 0 & 0 \\
\hline KELAUUNEE & 0 & D & 0 & D & 0 & 0 & 0 & 0 & 0 & 0 & 0 & 0 & 0 & 0 \\
\hline LA CAOSSE & o & 0 & o & 0 & 0 & $n$ & i & n & ס & 0 & B & to & 0 & n \\
\hline LA SALLE-1 & 195 & 296 & 0 & 197 & 197 & 0 & 199 & 36 & 36 & 0 & 36 & 16 & 0 & 36 \\
\hline LA SALLE-? & 195 & 196 & 0 & 197 & 197 & n & 198 & 36 & 36 & .1 & 36 & 36 & 0 & 26 \\
\hline LIMLRICK-1 & 0 & 220 & 221 & a & 222 & 222 & D & a & דه & fit & 0 & 40 & (0 & 0 \\
\hline LIMERICK-2 & 219 & 217 & o & 221 & 222 & 0 & 223 & 4n & 39 & 0 & 40 & th & 0 & 41 \\
\hline WIME YAMKEE & 0 & 0 & 0 & $\mathrm{~J}$ & 0 & 0 & 0 & D & 9 & 0 & 0 & 0 & 0 & 0 \\
\hline $\mathbf{X}$ GUTRE -1 & 193 & 0 & o & 0 & 0 & $n$ & 7 & 82 & נa & ! & 0 & 0 & J & in \\
\hline$\pi \quad C H \perp R E-2$ & 193 & a & 0 & u & 0 & 0 & D & 32 & 0 & 0 & 0 & 0 & 9 & 0 \\
\hline$M / O L A M D-1$ & 56 & 0 & 56 & 57 & 0 & 57 & J & 26 & 门 & .5 & 27 & D) & 27 & d \\
\hline YIOLAMD-2 & 56 & 56 & 0 & 5) & 5) & 0 & 57 & 26 & 26 & 0 & 27 & 27 & 0 & 27 \\
\hline MILLSTONE-1 & 0 & 0 & 0 & 0 & 0 & D & 0 & 0 & 0 & 7 & 0 & 9 & D & a \\
\hline पILLSTDNE-2 & 0 & 0 & 0 & 0 & 0 & 0 & D & $\mathrm{J}$ & $\eta$ & 0 & .1 & 0 & 0 & 0 \\
\hline MILLSTONE -3 & so & 56 & 0 & 57 & 57 & 57 & 57 & 26 & 26 & 3 & 26 & 26 & 26 & 26 \\
\hline ANTICELLO & 0 & a & 0 & a & 0 & 0 & 0 & 0 & 0 & 0 & 0 & 0 & 0 & 0 \\
\hline WINE MILE POLMT-1 & 0 & a & 0 & D & 0 & D & J & 0 & J & $\pi$ & $n$ & $n$ & ? & a \\
\hline NINE MILE POINTSZZ & 233 & $\mathrm{~J}$ & 235 & $\mathrm{~J}$ & 236 & 236 & 0 & 42 & D & 12 & a & 42 & s? & 0 \\
\hline WRTH AMHA-1 & 0 & a & 0 & a & D & ? & J & $n$ & 0 & 1 & $n$ & 0 & 1 & $a$ \\
\hline NORTH AMMA-Z & 0 & 3 & 0 & J & 0 & 0 & it & פי & 0 & $n$ & J & $n$ & 12 & 1] \\
\hline J)OKEE-1 & 3 & 0 & 0 & 1) & ? & 0 & a & $n$ & ] & .1 & g & in & 1 & n \\
\hline UCONEE - 2 & 0 & 3 & 0 & ] & 0 & 0 & $n$ & J & i] & n & 1 & ]. & $n$ & ה \\
\hline UCJMEE - 3 & 1 & 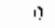 & 7 & 0 & 0 & n & 9 & $n$ & 1 & וי & ה & $B$ & $\eta$ & 0 \\
\hline DYSIER CREEK & 1) & .] & 0 & 3 & 0 & i) & 0 & 0 & i) & $n$ & .1 & 0 & $n$ & o \\
\hline PAL:SADES & $\mathrm{J}$ & 0 & a & ?) & $n$ & a & 1 & D & 7 & :) & $n$ & i) & 0 & n \\
\hline PALU AERDE-1 & 57 & 57 & 57 & 57 & $5 \mathrm{~A}$ & $5 \mathrm{H}$ & 58 & 25 & 25 & 15 & 25 & 25 & 25 & 25 \\
\hline 2ALO VERDE -2 & 56 & 5) & 97 & 57 & 58 & 58 & Sa & 24 & 25 & $\therefore 5$ & 25 & 25 & 25 & 25 \\
\hline JALU VERDE $=3$ & 37 & 57 & 57 & 58 & 58 & 58 & 58 & 25 & 25 & 9 & 25 & 25 & 25 & 25 \\
\hline PEACH BOTTIM-2 & 3 & 1) & is & ] & 0 & $n$ & 1 & n & ] & ) & ] & $n$ & b & ] \\
\hline DEACH 3ОTTOM-3 & 0 & נ] & u & J & ] & $\mathrm{u}$ & 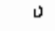 &. & 0 & 0 & v & J & 0 & $\Pi$ \\
\hline PERRY $=\mathrm{i}$ & J & 259 & 261 & ] & 261 & 261 & 了 & v & $5 ?$ & .94 & ] & 46 & $\triangle A$ & ن \\
\hline$P \subseteq k 8 \gamma-2$ & S\$ & $\mathrm{J}$ & 259 & 261 & 0 & 261 & 262 & 47 & 1) & 47 & 49 & a & 18 & 49 \\
\hline 3 ! LGR IM-1 & J & $n$ & U & ๙ & i) & ) & j & 1) & . & : & Ј & $n$ & $n$ & $\vec{J}$ \\
\hline POCNT $3 Е A C H-1$ & $v$ & J & 0 & 1 & ] & 0 & i) & J & n) & 1) & 0 & .7 & 0 & 0 \\
\hline गINF ВEACY-2 & 」 & 0 & $\mathrm{a}$ & a) & n & 10 & J & 1) & 1 & $\therefore$ &. & $n$ & 0 & ]. \\
\hline DAALRIE ISLANO-1 & 0 & 0 & D) & 0 & J & 0 & 0 & 3 & ] & ) & 3 & ] & י) & 0 \\
\hline DRALRIE [SLAMO-2 & 0 & 0 & i) & 1] & 1) & 9 & . & $n$ & 1 & "I & 1 & n & 1) & ] \\
\hline JUAO CITIES-I & u & '] & 0 & it & ] & ·] & D & J & fo & $\pi$ & 17 & . & J & n \\
\hline 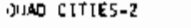 & i) & 0 & 13 & i) & D & 0 & $n$ & $D$ & 1 & $n$ & 7 & 1 & 1) & n \\
\hline TANCHO SECO-L & 0 & a & i) & 0 & 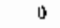 & $n$ & 0 & 3 & 0 & 0 & D & $n$ & :] & 0 \\
\hline RTVER BE NO-1 & 132 & 133 & 137 & 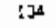 & 134 & 134 & 138 & 23 & 24 & $? 4$ & 24 & 24 & 24 & 24 \\
\hline XUB CKSON-Z & 了] & It & 0 & 9 & i & 7 & 1) & $\mathrm{J}$ & $D$ & 7 & $n$ & 0 & ? & ה \\
\hline SALEM-1 & 0 & 0 & 0 & g & וי & 1) & 1) & 1) & $\pi$ & $n$ & 1) & 9 & i) & \\
\hline
\end{tabular}


TABLE A.3. (contd)

\begin{tabular}{|c|c|c|c|c|c|c|c|c|c|c|c|c|c|c|}
\hline \multirow[b]{2}{*}{ REACTOR } & \multicolumn{7}{|c|}{ NSSEMLIES } & \multicolumn{7}{|c|}{ 而! m } \\
\hline & 014 & 2015 & 2016 & 2017 & 2018 & 2019 & 2020 & 2014 & 21915 & 2तार & 21ाT & 2018 & 205? & $202 \pi$ \\
\hline SALEM-2 & D & 0 & 0 & V & D & 0 & 0 & 0 & 0 & o & 0 & 0 & $B$ & 0 \\
\hline SAM OMOFRE -1 & 0 & 0 & 0 & 0 & 0 & 0 & a & 0 & 0 & 0 & n & 0 & 0 & $\pi$ \\
\hline SAN OMOFRE-? & 217 & 0 & 0 & 0 & 0 & 0 & 0 & 91 & 0 & 0 & o & 0 & 0 & n \\
\hline SAK ONOFRE-3 & 0 & 90 & 0 & 71 & 0 & 71 & 11 & 0 & 29 & 0 & 30 & D & 30 & 30 \\
\hline SEABRDOK-! & 56 & 56 & 57 & 57 & 57 & 57 & 57 & 24 & 24 & 24 & 24 & 24 & 24 & 24 \\
\hline SEABRDOK-? & so & 56 & 56 & 57 & 57 & 57 & 5) & 24 & 2 & 24 & 24 & 24 & 24 & 24 \\
\hline SEQUDYAH-I & $\mathbf{0}$ & 0 & 0 & 0 & 0 & 0 & 0 & 0 & 0 & 0 & 0 & 0 & 0 & 0 \\
\hline SEOUOYAH-Z & 0 & 0 & 0 & 0 & 0 & 0 & 0 & 0 & a & 0 & 0 & 0 & 0 & 0 \\
\hline SHOREHNH & 0 & 157 & 158 & 0 & 159 & 159 & 0 & 0 & 29 & $\boldsymbol{x}$ & 0 & 29 & 29 & 0 \\
\hline SOUTM TEXSS PRQJ-1 & 54 & 54 & 55 & 55 & 55 & 55 & 55 & 2 & $a$ & 30 & 30 & 30 & 30 & 30 \\
\hline SDUTH TEXAS 9R(O) -2 & s4 & se & 55 & 55 & 55 & ss & 55 & 29 & 29 & $x$ & $x$ & 30 & 30 & 30 \\
\hline 5T. LUCIE -1 & a & 0 & 0 & 0 & $a$ & 0 & 0 & 0 & 0 & 0 & 0 & $n$ & $\pi$ & ta \\
\hline ST. LUCLE+2 & 77 & 0 & 67 & 78 & 0 & 67 & 78 & 2 & 0 & 25 & 30 & 0 & 25 & 30 \\
\hline SUITER & 157 & 0 & 0 & 0 & 0 & 0 & 0 & 69 & 0 & 0 & $n$ & 0 & 0 & o \\
\hline SURRY $=1$ & 0 & 0 & 0 & 0 & 0 & 0 & 0 & a & 0 & D & 0 & 0 & n & n \\
\hline SURRY -2 & $n$ & 0 & 0 & 0 & 0 & D & 0 & 0 & 0 & a & D & 0 & n & 0 \\
\hline 5USOUEHANMA-1 & 219 & 220 & 0 & 221 & 222 & 0 & 223 & 39 & 39 & 0 & 39 & 39 & 0 & 39 \\
\hline SUSOUEHAMML- 2 & 219 & 0 & 221 & 222 & $n$ & 222 & 223 & 39 & 0 & 39 & 39 & t & 39 & 39 \\
\hline THREE MILE ISLARID-] & 0 & 0 & 0 & 0 & 0 & 0 & 0 & 0 & ก & 0 & a & n & 0 & 0 \\
\hline TRQJAN & 0 & 0 & 0 & n & 0 & th & 0 & in & 0 & 0 & $n$ & ก & 0 & 0 \\
\hline TURKE, POIMT-3 & 0 & 0 & 0 & 0 & 0 & 0 & 0 & 0 & 0 & 0 & 0 & 0 & 0 & $n$ \\
\hline TURKEY POINTAA & 0 & D & 0 & D & a & n & 0 & a & 0 & 0 & 0 & 9 & 0 & 0 \\
\hline VERMONT YAMKEE & 0 & 0 & 0 & 0 & 0 & 0 & 3) & 0 & D & 0 & 0 & 0 & a & a \\
\hline VOGTIE-1 & so & 56 & 56 & 57 & 57 & \$7 & 57 & 26 & 26 & 26 & 26 & $2 \pi$ & 26 & $2 f$ \\
\hline VOGTLE 2 & 56 & 56 & 57 & 57 & 57 & 57 & 26 & 26 & 26 & 26 & 26 & 28 & 26 & \\
\hline WAYERFORB-3 & 64 & 62 & 65 & 63 & 64 & 0 & 65 & 27 & 26 & $2 \mathrm{~B}$ & 27 & 27 & $n$ & $2 \mathrm{P}$ \\
\hline WATTS GAN-1 & 13 & 0 & 74 & 14 & 0 & 74 & D & 32 & 0 & 33 & 33 & a & 33 & 0 \\
\hline WATTS BAR-2 & 13 & 74 & 0 & 14 & is & 0 & 75 & 32 & 33 & 0 & 33 & 13 & 0 & 3 \\
\hline WNP - 1 & 56 & 56 & so & 57 & 57 & 57 & $\$ 7$ & 26 & $2 \pi$ & 26 & 26 & 26 & $2 \kappa$ & 26 \\
\hline WNP -? & 146 & 147 & โ & 148 & 148 & 148 & 149 & 27 & 27 & 27 & 27 & 27 & 27 & $?$ \\
\hline WNP - 3 & 66 & 67 & 67 & 67 & 67 & 67 & $\leftrightarrow$ & 28 & 29 & 29 & 29 & 29 & 29 & 20 \\
\hline WOLF CAEEK & 0 & 63 & so & 0 & 60 & 60 & $\pi$ & $n$ & 29 & $3 n$ & 1 & $3 n$ & $3 n$ & r. \\
\hline YAMXEE & 0 & 0 & D & 0 & 0 & 0 & 0 & J & $n$ & 7 & $n$ & 7 & $n$ & ? \\
\hline YELLDU CREEXX-1 & 0 & $n$ & 梋 & a & 79 & 78 & i & 0 & 33 & $3 ?$ & గ & $3 z$ & 12 & $\therefore$ \\
\hline YELLOH CAEEK-? & $n$ & 0 & 78 & 78 & 0 & 78 & $T \boldsymbol{R}$ & 33 & 0 & 33 & 37 & $n$ & 33 & 37 \\
\hline $210 \mathrm{M}-\mathrm{j}$ & 1 & n & B & n & 0 & 1 & r. & $n$ & B & ה & $r$ & ה & $n$ & $\therefore$ \\
\hline $260 k-2$ & 0 & 0 & 0 & 0 & $n$ & n & ה & $n$ & 0 & ח & $n$ & ז & n & x \\
\hline BRONSWICK-1 PLR $200 \mathrm{C}$ & I & 0 & D & n & $n$ & 7 & 0 & $n$ & n & 0 & n: & $n$ & חי & r \\
\hline BRUNSWICK-2 PHA POOL & 0 & 0 & 0 & 0 & a & 0 & 了 & is & j] & 口 & 0 & $\Pi$ & 1 & 1: \\
\hline MORR LS- GU & $u$ & 0 & 0 & 0 & $\eta$ & 0 & D & ? & 0 & 0 & $n$ & $?$ & $r_{1}$ & ר. \\
\hline MDRR I L-PLRR & 0 & to & J & 0 & 0 & 0 & 3 & $n$ & 0 & 0 & 0 & 0 & חִ & ? \\
\hline HEST YALLEY -BHA & 0 & 0 & 0 & 0 & 0 & 0 & 0 & $a$ & n & 0 & 0 & $a$ & $r:$ & $?$ \\
\hline WE 5† YALLEY-PNR & 0 & 0 & 0 & $\mathrm{~J}$ & 0 & 0 & 0 & 0 & 0 & 0 & $\eta$ & 0 & 3 & 0 \\
\hline 8W GENERIC & 6295 & 4652 & 6160 & 6318 & 5672 & 6090 & 7517 & 1161 & 856 & 1136 & 1153 & 1038 & 1112 & :315 \\
\hline PWR GENERIC & 3314 & 2856 & 3611 & 4146 & 4265 & 4168 & 4508 & 1งd! & 1668 & 1555 & 1787 & 1821 & 1786 & 1922 \\
\hline
\end{tabular}




\section{TABLE A.3. (contd)}

\begin{tabular}{|c|c|c|c|c|c|c|c|c|c|c|c|}
\hline & \multicolumn{11}{|c|}{ TOTALS } \\
\hline & 1983 & 1980 & I9BS & 1986 & 1987 & 1988 & 1999 & 1990 & 1991 & i99? & 1993 \\
\hline DLR NSEROLYY & 14114 & 1630 & 2310 & 2031 & 2526 & 3052 & 1092 & 3196 & 3212 & 3557 & 3594 \\
\hline 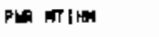 & 5953 & $\operatorname{los}$ & 1002 & a76 & 1099 & 1316 & 1311 & 1380 & 1388 & 1547 & 1568 \\
\hline BUA NSSERALY & $2315 ?$ & 2417 & 2531 & 3602 & 3499 & 4104 & 4128 & 4265 & 4764 & 2798 & 4864 \\
\hline gie mithe & $\$ 189$ & 416 & 453 & 647 & 628 & 743 & 743 & 760 & 858 & $n$ & g 78 \\
\hline TOTAL ASSEMELY & 37271 & 4047 & | I & 5633 & 6025 & 7156 & 7220 & 7483 & 7976 & 1855 & 8458 \\
\hline \multirow[t]{2}{*}{ 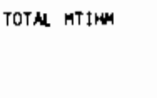 } & 20142 & 1125 & 1455 & 1523 & 5727 & 2059 & 2114 & 2346 & 2246 & 2321 & 2447 \\
\hline & 1994 & 1995 & 1996 & 1997 & 1998 & 1999 & 2000 & 2001 & -2002 & 2003 & \\
\hline PLAR ASSEMALY & 3932 & 3676 & 2031 & 4048 & 3735 & 4043 & 3955 & 2813 & 4163 & $\$ 319$ & \\
\hline Pun mikn & 1663 & 1604 & 1664 & 1763 & 1623 & 1765 & 1725 & 1676 & $: 920$ & 1888 & \\
\hline $\begin{array}{l}\text { ALR ASSERLIY } \\
\text { BHR RTIHM }\end{array}$ & $\begin{array}{l}5064 \\
930\end{array}$ & $\begin{array}{r}4950 \\
895\end{array}$ & $\begin{array}{r}5455 \\
\$ 60\end{array}$ & 5265 & $\begin{array}{r}5412 \\
975\end{array}$ & $\begin{array}{r}5240 \\
546\end{array}$ & $\begin{array}{l}8004 \\
1079\end{array}$ & $\begin{array}{r}2819 \\
870\end{array}$ & $\begin{array}{l}6559 \\
1180\end{array}$ & $\begin{array}{l}5965 \\
10808\end{array}$ & \\
\hline TOTAN ASSE maty & 8896 & 8626 & 9286 & 9333 & 9147 & 9283 & 9959 & 8632 & 10721 & 10284 & \\
\hline \multirow[t]{2}{*}{ TOTAL NTIH } & 2573 & 2499 & 2644 & 2717 & 2598 & 2711 & 2803 & 2546 & 9000 & 2975 & \\
\hline & 20104 & 2005 & 2006 & 2007 & 2008 & 2009 & 2010 & 2011 & $2 \pi n 12$ & 2013 & \\
\hline PWR NSSEMBLY & 4410 & 4777 & 4672 & $A B 2 B$ & 5606 & 6769 & 6114 & $\mathbf{s} 23$ & 6034 & 5a11 & \\
\hline 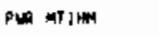 & 1941 & 2080 & 2053 & 2119 & 2442 & 2911 & 26R日 & 236A & 2687 & 2376 & \\
\hline BUA NSSEMBLY & 6077 & 7521 & 6485 & 9490 & 9677 & 10295 & 9278 & 10203 & $n 2 R$ & ? $\mathrm{KgP}$ & \\
\hline 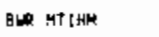 & 2098 & 1354 & 1175 & 1731 & 1567 & 1878 & 1519 & ? & 1418 & $! \$ 6$ & \\
\hline TOTAL NSEMTOLY & 10487 & 12298 & 11137 & 14318 & 14283 & 17054 & 14392 & 15828 & $137 \% 2$ & I3ina & \\
\hline \multirow[t]{2}{*}{ TarN Mr:H } & 3040 & 3439 & 3228 & 3850 & 4009 & 4843 & 4207 & 4366 & 4105 & 3792 & \\
\hline & 2914 & 2015 & 2016 & $20 ; 17$ & 2018 & $? 019$ & $202 !$ & & & & \\
\hline OLA ASSEMBLY & 6073 & 5697 & 5479 & 6054 & 6100 & 6:3? & 5192 & & & & \\
\hline PUR AT I HM & 2650 & 2380 & 2379 & 2628 & 2639 & 2853 & 2651 & & & & \\
\hline BLAR ASSEMBLT & 9206 & 7745 & $a b+1$ & 9020 & 9776 & वुसमी & 9987 & & & & \\
\hline BUR WTIHM & 1637 & 1516 & 1584 & 1643 & 1600 & 1509 & 1821 & & & & \\
\hline :0TA ASSEMBLY & 15279 & 13442 & 14120 & 15074 & 14884 & 14977 & 16170 & & & & \\
\hline TOTAL NTाM & 4337 & 3847 & 3963 & 4270 & 4239 & 4262 & 9482 & & & & \\
\hline
\end{tabular}




\section{TABLE A.4. Middle Case 1983 and Projected Inventories}

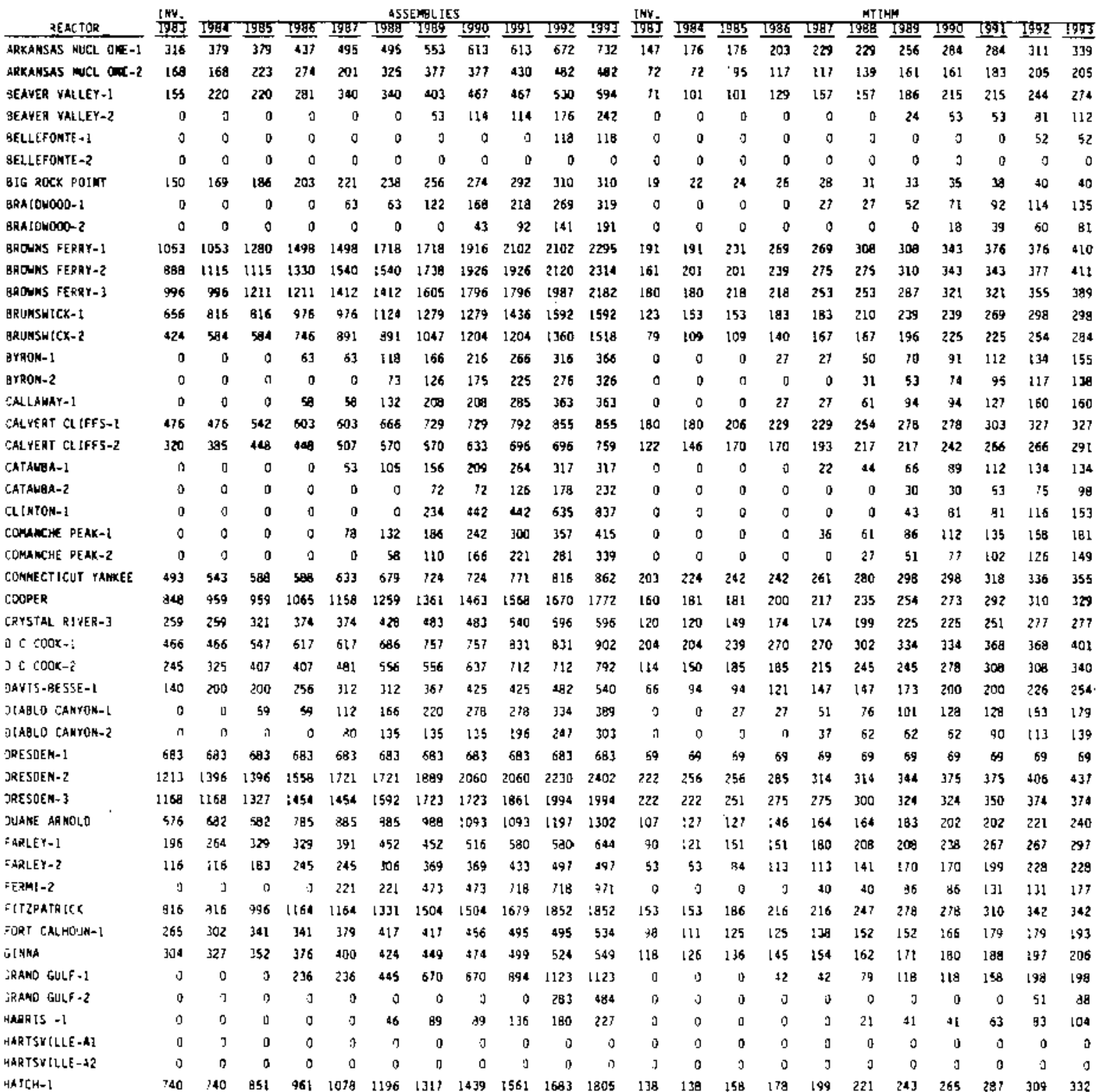




\section{IABLE A.4. (contd)}

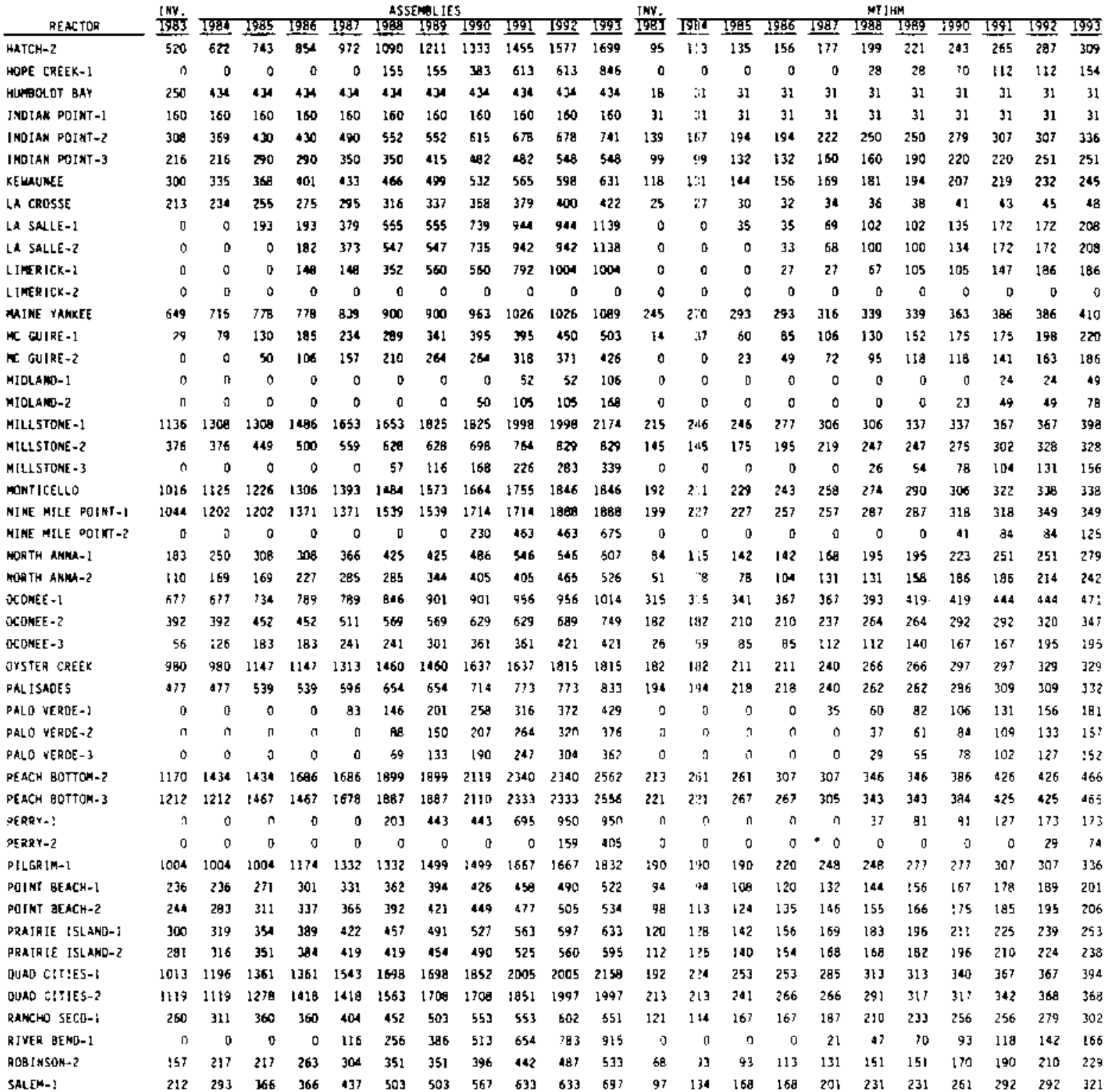


TABLE A.4. (contd)

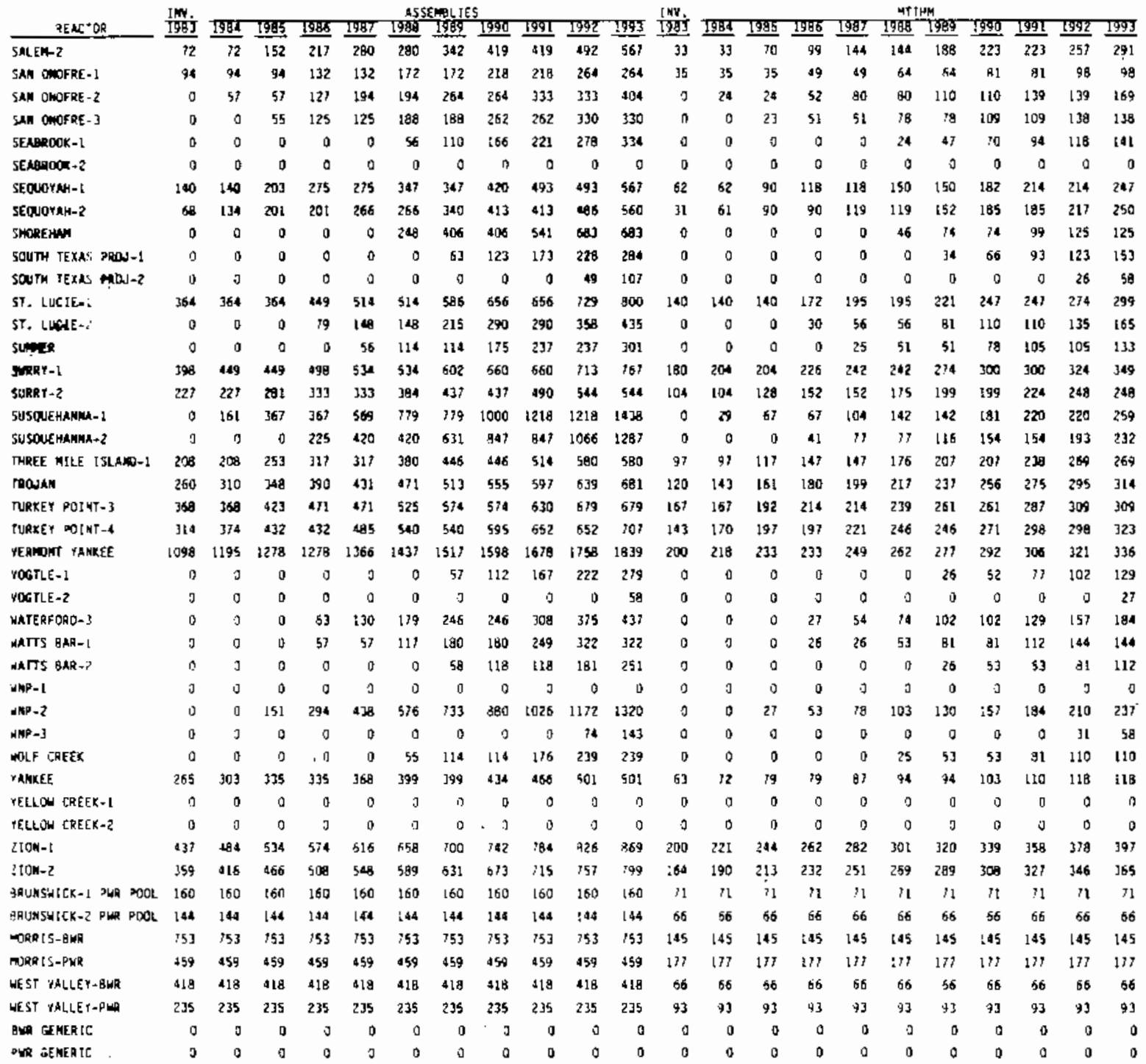


TABLE A.4. (contd)

A5SEMEL JES

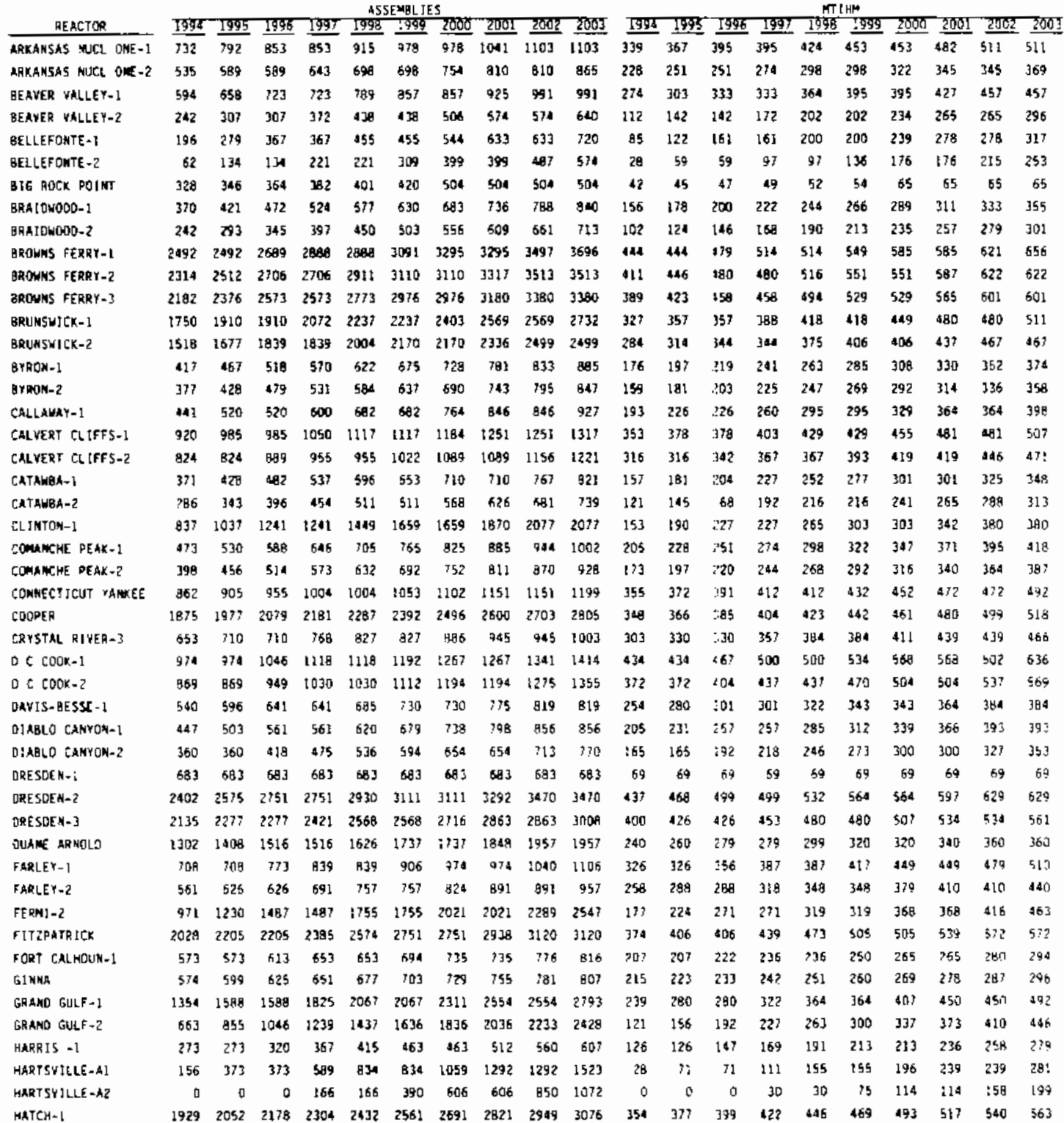


TABLE A.4. (contd)

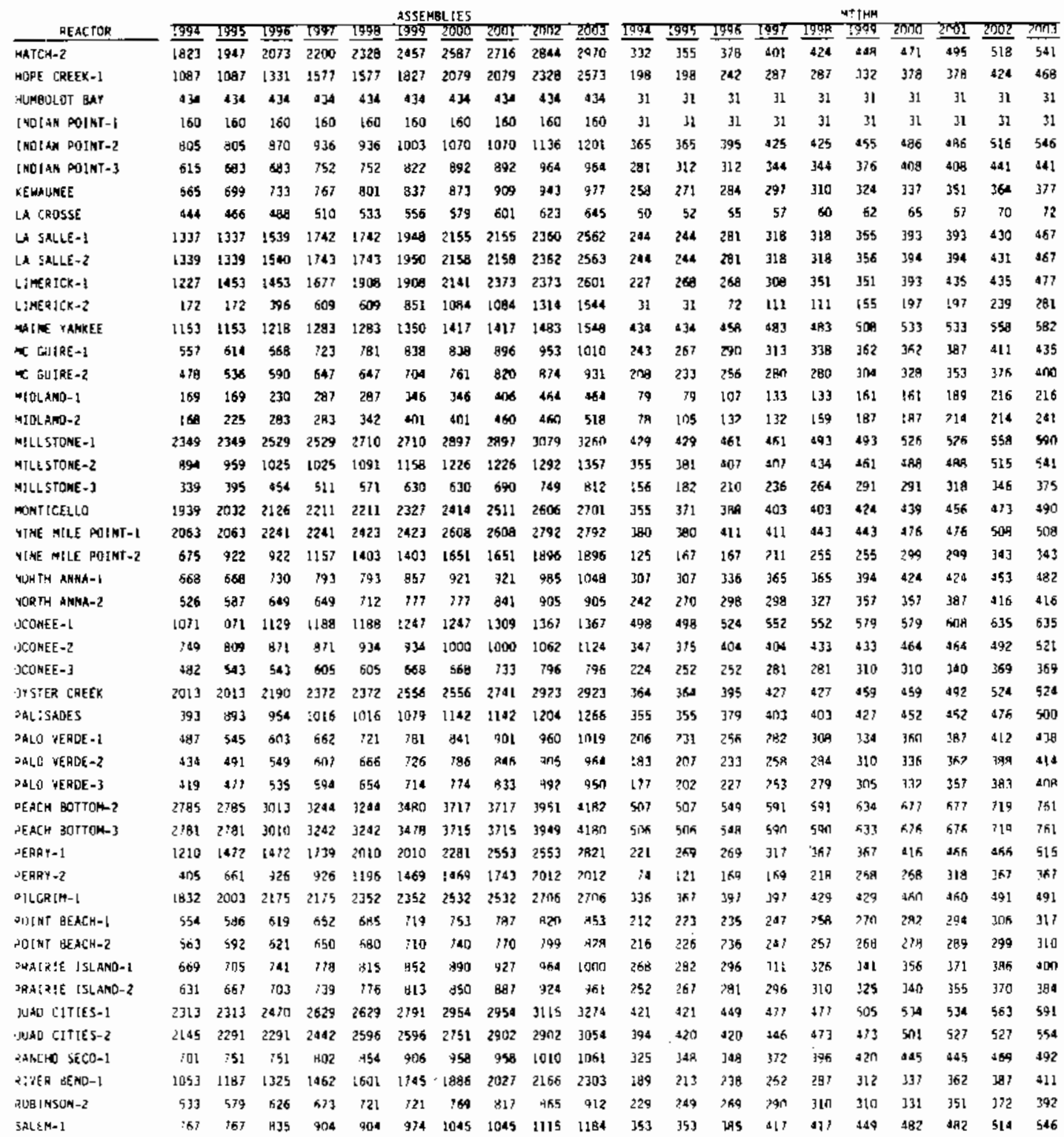


TABLE A.4. (contd)

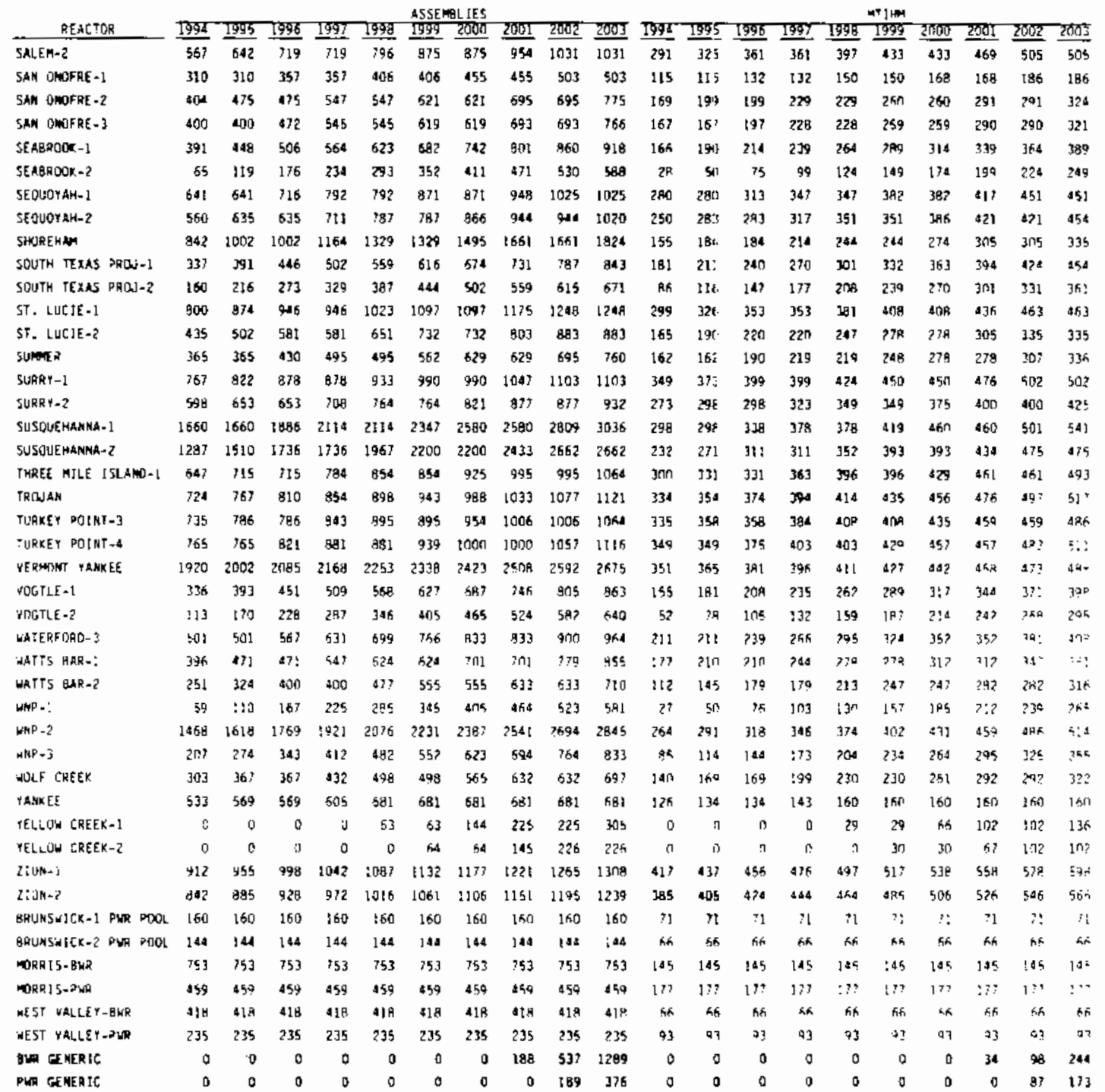




\section{TABLE A.4. (contd)}

\begin{tabular}{|c|c|c|c|c|c|c|c|c|c|c|c|c|c|c|c|c|c|c|c|c|}
\hline \multirow[b]{2}{*}{ EACTRE } & & \multicolumn{10}{|c|}{ IHM } \\
\hline & 804 & pas & 2005 & נ07 & & $\Rightarrow$ & 2010 & 2011 & द्राश्र & 2013 & 2004 & 8005 & 2006 & 007 & 명 & 2009 & 2010 & $0 ! !$ & 012 & 013 \\
\hline KANSAS WUCL ONE-1 & 164 & 225 & 1225 & 1298 & 1347 & 1347 & 1524 & 1524 & 1524 & 1524 & 539 & 588 & 560 & 596 & 624 & 624 & 706 & in6 & 706 & 706 \\
\hline KAMSASS mUCL D & 919 & 919 & 973 & 127 & 1027 & OQ8O & 32 & $\lfloor 132$ & 184 & 361 & 392 & 392 & 415 & 438 & 438 & 481 & 483 & 483 & 505 & 520 \\
\hline AYER YALLEY $Y=1$ & 058 & 121 & 122 & 86 & 1250 & 250 & 314 & $\{4\} \mid$ & 1471 & 147 & क्षी 7 & 517 & 517 & 547 & 577 & 577 & 16 & 679 & 679 & 579 \\
\hline AYER VALLEY-2 & 705 & 305 & 770 & $B 35$ & B35 & 999 & 962 & 962 & 025 & 1009 & 326 & 326 & 358 & 85 & 185 & ه15 & 444 & 844 & 473 & 503 \\
\hline LLEFONTE-1 & 베 & a0t & 393 & 13 & 979 & 1063 & 1083 & 1147 & 1231 & 231 & 355 & 355 & 393 & 393 & 431 & $4 \times 8$ & 148 & Sก5 & 542 & 42 \\
\hline 11EFFNTE-2 & 574 & 661 & 661 & 747 & 832 & 32 & 416 & 1000 & 000 & 1004 & 253 & 292 & 292 & $33 n$ & 367 & 367 & 404 & 441 & $\Delta_{1}$ & 479 \\
\hline$R \cdot J C X$ Pa & 504 & 504 & 504 & 504 & 504 & 504 & Sod & 504 & 504 & $\sin 4$ & 65 & א5 & 65 & 65 & 55 & 65 & 65 & 65 & 85 & 55 \\
\hline $1000-1$ & 542 & 944 & 995 & 1048 & 1097 & 1147 & 1197 & $124]$ & 1297 & 1347 & $3 n$ & 399 & 421 & sA? & 464 & 285 & 506 & 527 & 548 & 568 \\
\hline $000-2$ & 165 & 816 & 867 & 918 & 969 & 019 & 1069 & 1119 & 1169 & 1219 & 323 & 305 & 367 & 368 & $\$ 10$ & 431 & 452 & a 13 & 494 & 515 \\
\hline FERRY & 3696 & 18997 & 97 & 4097 & 1851 & 4861 & 961 & 161 & 4861 & 4851 & 656 & 691 & 726 & 126 & 6 & 860 & 60 & 860 & 860 & 60 \\
\hline DWHS $f$ & 14 & 908 & 909 & 100 & 4308 & 5072 & 5072 & 5072 & 5072 & 5012 & 657 & की! & 691 & 726 & 761 & 395 & 895 & 895 & 595 & 395 \\
\hline $605 F$ & 78 & 3776 & 176 & 912 & 4168 & 4160 & 932 & 1912 & 1932 & 932 & 636 & 671 & 671 & 105 & 740 & 740 & 815 & d75 & 875 & 375 \\
\hline ILK-I & 194 & 2894 & 055 & 3215 & 3215 & 3374 & 3533 & 4093 & 4093 & 4093 & 541 & 541 & 571 & 601 & $\delta 01$ & 631 & 61 & 765 & 765 & 365 \\
\hline $1 C K-2$ & 661 & 2823 & 423 & 984 & 3143 & 3143 & 3302 & 2862 & 3962 & 3862 & 498 & 528 & 528 & 558 & 560 & 580 & 618 & 122 & 122 & 122 \\
\hline & 937 & 989 & 40 & 1041 & 1142 & {$[193$} & 1243 & 1293 & 1343 & 1393 & 396 & 418 & 440 & 461 & 483 & 504 & 26 & 447 & S68 & 589 \\
\hline & 899 & 951 & 102 & 1053 & lias & 1154 & 1204 & 1254 & 1304 & 1354 & 120 & 402 & 424 & 445 & 467 & 48 & 509 & 530 & 31 & 72 \\
\hline$y=$ & 07 & 107 & B) & 167 & 1167 & 1245 & 322 & 322 & 399 & 476 & $\$ 32$ & 432 & 66 & 500 & 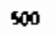 & 513 & & 5 & 598 & $3 t$ \\
\hline 16 & 32 & 1382 & 147 & 1512 & 1512 & $15 \%$ & 1794 & 1794 & 1794 & 1794 & 532 & 532 & 5 & 583 & 583 & 600 & 13 & 93 & 693 & 693 \\
\hline $\mathrm{c}$ & 21 & 96 & 51 & 351 & 1416 & 1481 & 1698 & 1698 & 1698 & 1698 & 471 & 496 & 1 & 521 & 17 & 572 & 57 & 857 & 657 & 557 \\
\hline & 879 & 932 & 989 & {$[043$} & 1088 & 1151 & 1151 & 1204 & 1257 & 1314 & 312 & 395 & 419 & 442 & 465 & ง89 & 488 & 510 & 532 & 557 \\
\hline $4-2$ & 93 & 850 & 905 & 905 & 960 & 1014 & 2067 & 1122 & 1174 & 229 & 336 & 360 & 183 & 393 & 407 & 430 & 152 & 475 & 497 & 521 \\
\hline$k-1$ & 292 & 187 & 2487 & 2690 & 2892 & 2992 & 3090 & 3288 & 3289 & $34 A 7$ & 417 & 455 & 455 & $\Delta 92$ & 529 & $5 \%$ & 15 & $n$ & 501 & 18 \\
\hline MACHE $P$ & 60 & 18 & 76 & 234 & 1292 & {$[349$} & 1405 & 1461 & 1517 & 1573 & 41 & 465 & $\angle 8 B$ & 511 & 535 & 558 & 590 & 3 & 676 & 48 \\
\hline $\operatorname{ranch} P$ & 86 & 44 & 102 & 159 & 1216 & $127]$ & 399 & 1385 & 1442 & 1498 & 410 & 0.36 & 457 & $2 B \Omega$ & 503 & $\$ 26$ & 49 & 1 & 194 & 17 \\
\hline tcus & 147 & 104 & 404 & 104 & 1404 & 1404 & $140 \mathrm{~d}$ & 1404 & 1404 & $14 \cap 4$ & 512 & 576 & 576 & 576 & 576 & 576 & 576 & 15 & $5 / 6$ & 576 \\
\hline PPE & 06 & נם & Wet & 09 & 3310 & 3858 & 858 & 3858 & 3858 & 78se & 536 & 955 & 57 & 592 & 610 & 711 & & 711 & 11 & $\because i 1$ \\
\hline NL $d$ & $16 i$ & 61 & 19 & 1136 & 1176 & 1353 & 1353 & 1353 & 1353 & 1353 & 492 & 992 & 519 & 546 & 546 & 6. $2 y$ & 628 & 528 & $52 B$ & 628 \\
\hline $0 k=$ & 14 & $d ?$ & SA & 559 & 1631 & 1303 & 1896 & 1996 & 1896 & 1896 & 636 & 569 & 702 & $? 72$ & 31 & 768 & 857 & 7 & 857 & 457 \\
\hline$k-2$ & 55 & 1435 & 15 & 515 & 1594 & 1673 & 1866 & 1866 & 1866 & $! 866$ & 569 & 602 & 14 & 634 & 565 & 698 & 76 & 75 & 776 & 776 \\
\hline 然 & 863 & 907 & 707 & 750 & 40 & 993 & $: 035$ & 107 & 1077 & 1254 & 405 & $\$ 25$ & 5 & 446 & 466 & 466 & 5 & 15 & 505 & 588 \\
\hline $2000 \mathrm{C}$ & 714 & 473 & 1030 & 1087 & 1057 & 1144 & 1200 & 1255 & 1310 & 1367 & 420 & 447 & $3 / 3$ & 499 & 499 & 525 & 1 & 576 & 001 & 628 \\
\hline 0 & 24 & 305 & 943 & IAl & 1000 & 1056 & 1113 & 1168 & 1225 & 1225 & 381 & $\$ 06$ & 433 & 431 & $\$ 59$ & s45 & 1 & 36 & 562 & 562 \\
\hline$U E N=1$ & 83 & 83 & 633 & 683 & $5 \times 3$ & 683 & 683 & 683 & ถ⿻3 & 683 & 69 & 59 & hy & 39 & $A$ & 69 & 3 & F & 0 & 59 \\
\hline$M-2$ & 46 & 22 & 22 & 46 & 546 & 9456 & 546 & 4546 & 4546 & $\$ 46$ & 660 & 692 & 692 & $x 22$ & +22 & $d 22$ & 2 & 22 & 922 & 22 \\
\hline EN- $]$ & & $\$ 2$ & 3296 & 4020 & 4020 & 4020 & 1020 & 4020 & 4020 & 4020 & $f$ & 587 & & $14 t$ & ist & 747 & 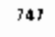 & 17 & $? \mathrm{~s}$ & 847 \\
\hline AME ARMO & 65 & 73 & 2173 & 2280 & 2386 & 2186 & 2492 & 60 & 2860 & 2860 & 380 & 399 & 9 & 419 & 478 & \& 早 & 5 & 25 & 525 & 25 \\
\hline$r=$ & & 71 & 1276 & 1236 & 1301 & 1365 & 1365 & 1429 & 1493 & 1493 & 510 & 540 & 570 & 570 & אח & 629 & 9 & 9 & ด.: & $8 B$ \\
\hline$E Y=?$ & 22 & 122 & $10 \mathrm{~B} 7$ & .52 & 1152 & 216 & 279 & 199 & $1] \Delta 2$ & 1499 & 470 & $47 n$ & 5 & 530 & 530 & 559 & A & ap & 17 & 39 \\
\hline & 4 & 10 & $\$ 10$ & 10 & 1070 & 3325 & 3575 & 3576 & 1831 & 3831 & 483 & $5 ! 1$ & 11 & $55 \mathrm{~A}$ & $3>B$ & की & & 50 & 9 & 7 \\
\hline als & 300 & 3400 & $34 \mathrm{ad}$ & 3658 & 18.35 & 3935 & जाए & 4572 & 4572 & $45 \pi 2$ & 005 & 539 & 678 & 670 & 803 & $7 \times 3$ & 735 & 937 & 837 & 啫 \\
\hline FüRt & & 456 & 396 & x96 & +36 & $! 069$ & 069 & 1069 & 1069 & 189 & 294 & उतg & 3 & 323 & 337 & J月4 & 4 & 14 & 84 & 94 \\
\hline & & & 15 & 1006 & 1006 & 1006 & 006 & 1006 & 1006 & ! 1006 & 05 & 314 & 32.4 & फ & in & 166 & 6 & 368 & 56 & $36 \hbar$ \\
\hline vithliti & & 30 & 67 & 15192 & 3502 & 135 & 3964 & 3964 & 4193 & 1423 & 534 & 534 & & 517 & 1 & $55 B$ & & A & 39 & 84 \\
\hline$i$ & 22 & $2 B 16$ & 3009 & 3202 & 3394 & ه853 & $3 n$ & 3959 & 4148 & 4335 & 481 & \$1) & 552 & SQR & 523 & 558 & 2 & $: 27$ & हु] & 96 \\
\hline $2-4$ & & 554 & 701 & 146 & 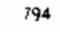 & d40 & 840 & 306 & 432 & 978 & 301 & 301 & & 244 & .265 & 786 & & $\triangle C D B$ & 429 & 50 \\
\hline & & 47 & 1912 & 2196 & 2196 & 2418 & 2636 & 2536 & 355 & 3074 & 321 & 321 & 22 & 403 & 403 & 444 & 3 & SR3 & 23 & 63 \\
\hline & & 94 & 1526 & 1524 & 170 & 1969 & 1969 & & 408 & (4) & $19 y$ & 240 & 2 & 292 & 322 & 362 & $\vec{a}$ & 02 & $9 s 2$ & 42 \\
\hline $216 \mathrm{CH}=1$ & 22 & 28 & 53 & 78 & 3702 & 926 & 196 & 786 & 385 & 196 & 86 & $\mathbf{a}$ & 632 & 54 & 575 & 100 & 802 & 302 & 802 & $1+12$ \\
\hline
\end{tabular}




\section{TABLE A.4. (contd)}

\begin{tabular}{|c|c|c|c|c|c|c|c|c|c|c|c|c|c|c|c|c|c|c|c|c|}
\hline \multirow[b]{2}{*}{ REACTOA } & \multicolumn{10}{|c|}{ ASSEMLIIES } & \multicolumn{10}{|c|}{ 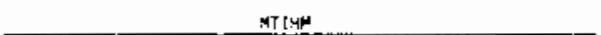 } \\
\hline & D4 & 85 & 2006 & 27 & & & 2010 & 2011 & 2012 & 2013 & 2001 & 2005 & 3006 & 2007 & & 2009 & 010 & $201 !$ & 012 & 6] \\
\hline $\mathrm{CH}-2$ & 196 & 3222 & 3347 & 3472 & 3596 & 3719 & 2840 & 3962 & 4084 & 4644 & 564 & 597 & 610 & 632 & 55 & 577 & 699 & 122 & ?.44 & 46 \\
\hline SEEEK-I & 73 & 818 & 3061 & 61 & 3303 & 3541 & 3541 & $\eta 7$ & 014 & 14 & 468 & 513 & 597 & \$\$1 & 1 & s4a & 5.44 & $6 \mathrm{~d} 7$ & 730 & 30 \\
\hline MBULOT HAY & 434 & 434 & 434 & 434 & 434 & 434 & 434 & 434 & 434 & 434 & 31 & 31 & 31 & 31 & in & 11 & 11 & ו & 1 & 31 \\
\hline MDIAN PUIN & 160 & 160 & 160 & 160 & 160 & 160 & 160 & 160 & 160 & 150 & 36 & 31 & 31 & $3 t$ & 31 & 31 & 31 & 31 & 31 & $3 t$ \\
\hline IMUJAN POLNT-Z & 1201 & 1260 & 1331 & 1524 & 1524 & 1524 & 1524 & 1524 & 1524 & 1524 & 546 & 575 & 605 & 693 & 593 & 593 & 693 & 593 & 693 & 693 \\
\hline [NOIAN $90[N T+3$ & 32 & 1099 & 1099 & 1169 & 1235 & 1235 & 1428 & 1428 & 1428 & 1428 & $47 ?$ & 503 & 503 & 535 & 565 & 565 & 551 & 651 & 651 & $\delta 51$ \\
\hline WALUEE & 111 & 1045 & 1079 & 15I & 1147 & 1268 & 1269 & 1268 & 1268 & 1268 & 390 & 403 & $\$ 16$ & 430 & 43 & 189 & 489 & $\angle 89$ & 289 & 489 \\
\hline CROSSE & 717 & 717 & 317 & $M$ & 717 & 117 & 717 & 117 & 717 & 717 & sa & 80 & 80 & 80 & 80 & 80 & do & (a) & 80 & 30 \\
\hline$L E-1$ & 2562 & 2164 & 2964 & 2964 & 3163 & 3359 & 3359 & 3553 & 3748 & 3748 & 167 & sod & 540 & 540 & 1 & 612 & 512 & 648 & 693 & 93 \\
\hline LLE-2 & 63 & 2764 & 2964 & 2964 & 3163 & 3358 & 1358 & 3552 & 3741 & 3747 & 467 & 504 & $\$ 40$ & 540 & $\eta$ & 612 & 512 & 547 & 93 & 683 \\
\hline MERICK-L & 27 & 2827 & 3053 & 3277 & 3277 & 3499 & 311 & 3717 & 3936 & 156 & \$18 & 518 & 559 & 600 & 00 & 640 & 580 & 680 & 20 & 60 \\
\hline MER ICK -2 & 544 & 2768 & 1993 & 1993 & 2217 & 2417 & 2037 & 2656 & 2876 & 2876 & 282 & 322 & 363 & 363 & 03 & 443 & 443 & 4A3 & 523 & 23 \\
\hline TKE YAHIKS & 548 & 1613 & 1679 & 1678 & {$[743$} & 1960 & 1960 & 1960 & !960 & 1960 & 582 & 606 & 631 & 631 & 655 & 137 & $: 37$ & $? 37$ & 37 & 37 \\
\hline UJRE- & 64 & 122 & 1176 & 1230 & 1285 & 1285 & 1338 & 1393 & 1446 & 1499 & 458 & 482 & 505 & 588 & 2 & 552 & 574 & 597 & 520 & 12 \\
\hline LU:RE-2 & 986 & 1041 & 1096 & 1096 & 1150 & 1205 & 1257 & 1312 & 1364 & 1416 & 924 & 447 & 470 & 470 & $\$ 93$ & 516 & 518 & 562 & sed & 606 \\
\hline $100-1$ & 522 & 522 & 580 & 637 & 837 & 693 & 693 & 149 & 805 & 80 & 203 & 243 & 270 & 296 & 6 & 323 & 123 & 349 & 75 & 15 \\
\hline $40-2$ & 576 & $b 76$ & 634 & 691 & 691 & 798 & 804 & 304 & 360 & 860 & 268 & 268 & 295 & 322 & 22 & 348 & $3 / 4$ & $3 / 4$ & 100 & Q \\
\hline LSTOME-1 & 260 & 3440 & 3440 & 4020 & 4020 & 4020 & 4020 & 4020 & 020 & 020 & 590 & 622 & 622 & 124 & 124 & 324 & 724 & 724 & 24 & 24 \\
\hline STUME-2 & 23 & 1423 & $: 448$ & 1552 & 1617 & $(6)]$ & 1682 & เ899 & 1894 & 1899 & 568 & 568 & 594 & 620 & 546 & EA6 & 672 & 759 & 159 & 59 \\
\hline STUHE -3 & 60 & 422 & y60 & 37 & 1094 & 1094 & 1150 & 1206 & 1262 & 131 & 397 & 426 & 452 & 479 & 505 & 505 & 531 & 557 & 582 & 08 \\
\hline ICELLD & '46 & 2890 & 2943 & 2983 & 3467 & 3467 & 3467 & 3467 & 3467 & 346 & 507 & 523 & 540 & 540 & 25 & 625 & 625 & 625 & 525 & 25 \\
\hline NE MILE P & 172 & 2912 & 3504 & 3504 & 3504 & 3504 & $150 \mathrm{~N}$ & 3504 & 3504 & 3504 & 560 & 540 & 634 & 54 & 34 & 634 & 634 & 634 & هر & 34 \\
\hline MILÉ & 3) & 2378 & 2378 & 617 & 2617 & 2853 & 2953 & 3085 & 318 & 3.a & 187 & $\$ 30$ & 430 & 973 & 3 & 15 & 15 & 57 & 94 & 99 \\
\hline RTH AMNA-I & 48 & +10 & 1171 & 1171 & 1233 & 1293 & 1293 & 1353 & $: 510$ & 151 & 982 & 511 & 539 & 539 & 567 & 595 & 595 & 622 & 95 & 95 \\
\hline HTH $2 \times 1 \times$ & 167 & 1029 & 1029 & 1090 & 1152 & 1152 & $: 212$ & 272 & 429 & 1429 & 445 & 4ij & 413 & 501 & 530 & 530 & 557 & 585 & 57 & 57 \\
\hline$F=1$ & 25 & 1425 & 1483 & 1541 & $|54|$ & 1718 & {$[7: 3$} & 1718 & 719 & $71 \mathrm{R}$ & 662 & 562 & 689 & 715 & 715 & วด̣a & iqR & 798 & 99 & 98 \\
\hline-2 & 24 & 86 & 186 & ८ $24 A$ & 1425 & 1425 & 1425 & 1025 & 1425 & 1425 & 521 & 550 & 550 & 579 & 561 & 661 & 561 & á 61 & 61 & 61 \\
\hline$E-3$ & 59 & 920 & 920 & 981 & צHI & 1158 & 1158 & 1158 & 1158 & 1158 & 398 & 1?) & 427 & 455 & 455 & 537 & 537 & 537 & 537 & 37 \\
\hline & 05 & 65 & 3665 & 3655 & 3665 & 3665 & 3665 & 3665 & 3565 & 366 & $\$ 56$ & 555 & 655 & 655 & 5 & 655 & 555 & 655 & 55 & 55 \\
\hline DES & 56 & i327 & 1388 & 1386 & 1592 & :592 & 1592 & $: 592$ & 1592 & 1592 & $5 \cap n$ & 523 & 547 & sat & 27 & 527 & 6,7 & 627 & $57 ?$ & 2) \\
\hline & 7 & 35 & y3 & 1251 & 1 & 1386 & 122 & $! 478$ & 5 & 159 & 4 & 4 & 4 & 39 & 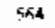 & 9 & 19 & 38 & 5.3 & คถ \\
\hline VERQE & 222 & !08: & 1139 & 1197 & $: 255$ & 1313 & 1369 & 1425 & $14 k 2$ & 253 & 439 & 465 & $19 \pi$ & $5 ! 5$ & 541 & 566 & 590 & $6 ! 5$ & 540 & 665 \\
\hline YEAUE-3 & 344 & 1067 & 1125 & 1183 & 1241 & {$[29]$} & 1353 & 1409 & 1456 & 1523 & 434 & \pm 57 & 285 & 510 & 5 & 5 & 584 & 609 & 633 & 358 \\
\hline$\theta$ & $\theta 2$ & 12 & $164]$ & 4641 & 4870 & 5634 & 5634 & 5634 & 56.34 & 563 & 161 & मำ 3 & 245 & 345 & 387 & 1026 & ח26 & 1026 & 026 & $2 \ln 26$ \\
\hline ACH DOTTOM-3 & 80 & 4410 & 46.39 & 4619 & 4868 & 5632 & 5632 & 5632 & $\$ 632$ & 5632 & $: 61$ & 703 & 244 & d44 & ; & 1025 & $! 025$ & 1025 & 1025 & i025 \\
\hline & & 35 & ]350 & 3614 & 3614 & 387 & 1132 & 4132 & 13049 & 4 the & 563 & 563 & 611 & i59 & 9 & in? & 154 & 154 & 800 & 4.9 \\
\hline$r-2$ & 271 & 2543 & 2543 & 2806 & 3066 & 2065 & 3323 & 1580 & 3580 & $3 \mathrm{~K} 39$ & $\operatorname{sis}$ & 459 & 959 & 507 & 555 & 555 & ו & 548 & 64 & 696 \\
\hline$J^{M}-\mathbf{i}$ & 5 & J3s3 & 3453 & 3225 & 5225 & 3605 & 3805 & 3805 & 3845 & 380 & 522 & 3 & 3 & 觓3 & & 96 & 6 & 85 & 586 & $\partial \delta$ \\
\hline$I S E A C H$ & ד' & $+1 y$ & 152 & tas & 1106 & 1106 & 1106 & 1106 & 1106 & 1106 & 329 & $j a l$ & 352 & 364 & 407 & atid 7 & 107 & 407 & 207 & 107 \\
\hline $\operatorname{GEACH}$ & 57 & 986 & 315 & 944 & 473 & 1094 & 1094 & 2004 & 1094 & 109 & 320 & 230 & 340 & 351 & 36.1 & $\$[1 / 4$ & 404 & 404 & 64 & 04 \\
\hline$A$ Lale [ & 036 & 12 & 1100 & 1144 & 1180 & $130 !$ & 1301 & 1301 & 1301 & 1301 & $\$ 14$ & 429 & 443 & $4 \$ B$ & 472 & 520 & 520 & 520 & 520 & 520 \\
\hline$\alpha$ LRIE ISLANU & 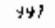 & 133 & $\operatorname{lu} 6 y$ & 1105 & 1141 & $: 262$ & 1262 & $: 262$ & 1262 & 1202 & 399 & 413 & 28 & 442 & 6 & 15 & 55 & 05 & 05 & 50) \\
\hline D & 174 & 32 & 3590 & 3590 & 4314 & 9310 & 4314 & 4364 & 4314 & 4 & 591 & 818 & 6 & 646 & 4 & 74 & 4 & 74 & 7 & 74 \\
\hline$A$ CIY!ES-Z & 05 & 3205 & 3353 & $40 \%$ & solt & 4.77 & 4077 & 417 & $40) 7$ & solt. & 581 & 581 & 607 & 134 & ?] & 134 & 34 & 134 & Iযa & 734 \\
\hline 105 & 12 & 1163 & 163 & 1214 & 1265 & 1442 & 1442 & 1442 & 1942 & 442 & $\$ 16$ & 540 & 540 & 563 & ? & 669 & 569 & 669 & 89 & 69 \\
\hline & 43 & 2580 & 16 & $2 B 52$ & 2940 & 3124 & 3256 & 3388 & 15 & 365 & & $\$$ \$ & & $5 n$ & 53 & 56 & & n02 & $62 \hbar$ & $55 \pi$ \\
\hline Rotingson-2 & & 359 & 1006 & 163 & $1: 63$ & 116 & 1163 & 1163 & 3 & 163 & 32 & A! 3 & 133 & 5110 & 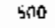 & to & 0 & $\sum n 0$ & $\sin \pi$ & סू \\
\hline ALEM-I & 站4 & 1253 & 321 & 1321 & 189 & 1582 & $6 B 2$ & 1582 & $15 P 2$ & $! 582$ & $5 \Delta 5$ & 39 & $n$ & fin & 541 & : & $73 n$ & 73 & חני & $3 c$ \\
\hline
\end{tabular}


TABLE A.4. (contd)

\begin{tabular}{|c|c|c|c|c|c|c|c|c|c|c|c|c|c|c|c|c|c|c|c|c|}
\hline \multirow[b]{2}{*}{ REALTOA } & \multicolumn{10}{|c|}{ ASSEMBLL IES } & \multicolumn{10}{|c|}{ ला [म" } \\
\hline & $\theta(0)$ & 2005 & 2006 & 2000 & 200 & 30109 & $\pi 0$ & 2011 & 2012 & 2013 & Tn] & 2005 & $200 \pi$ & not & 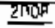 & 2/lk9 & गु? & 201 & 012 & $\pi I 2$ \\
\hline LEM-2 & 100 & 1185 & 1205 & 1260 & 1335 & 1375 & 28 & 2528 & 1528 & 528 & 540 & 575 & 575 & ถิग & 64 & 6.44 & 133 & 733 & 733 & $13 \pi$ \\
\hline N OMOFRE-1 & 551 & 700 & $+\infty$ & 708 & 708 & 708 & 308 & 700 & 708 & 708 & $20 \mathrm{~s}$ & 2 & 52 & 262 & 262 & 262 & 62 & 262 & $?$ & 262 \\
\hline IN JWOFRE-Z & 46 & 834 & $\$ 13$ & 913 & $98 \mathrm{~A}$ & 984 & 1054 & 1054 & 1124 & 1124 & 349 & 49 & 382 & 2B2 & 412 & 812 & el & 441 & 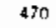 & (7) \\
\hline IN JMOFAE & 706 & 838 & 838 & 910 & 910 & 981 & 981 & $105 i$ & 1051 & 121 & 321 & ד & 351 & 391 & 31 & 412 & 11 & 440 & $84 \pi$ & 469 \\
\hline$A B H C O X-1$ & 976 & 1074 & 092 & 149 & 206 & 1262 & 318 & 1374 & 430 & 485 & 413 & 18 & $46 ?$ & 487 & 21 & 534 & 58 & 582 & 606 & 29 \\
\hline ARROOK - & $6+6$ & 304 & 62 & 19 & 876 & 933 & 389 & 1045 & 1101 & 157 & 274 & B & 23 & 347 & 3 & 395 & 419 & 442 & 466 & 90 \\
\hline OUVYAht $=1$ & 201 & 117 & 117 & 1252 & 1327 & 1327 & 1402 & 2402 & 1595 & 1595 & 485 & 8 & SiB & 552 & 85 & 585 & 618 & 618 & tou & 04 \\
\hline QUOYAM-Z & 96 & 1096 & 171 & 1171 & 1246 & 1320 & 1320 & 1513 & 1513 & 1513 & 480 & 988 & 521 & 521 & $\$ 55$ & 580 & 588 & 673 & 673 & 673 \\
\hline AEMAM & 1986 & 986 & 48 & 09 & 2309 & 2468 & 24 & 2624 & 2790 & 3) & 364 & 4 & 14 & 426 & 24 & 453 & 281 & 19] & 10 & 39 \\
\hline OUTH TEXNS PROS & ag9 & 955 & 011 & 1066 & 1121 & 1175 & 1229 & 1283 & 1331 & 1391 & 484 & if & 14 & 574 & 04 & 633 & 62 & 391 & 20 & 9 \\
\hline UTH TEXAS & 327 & 793 & 839 & 894 & 949 & 1003 & 057 & 1111 & 1165 & 1219 & 391 & 22 & 452 & 481 & 511 & 540 & 569 & 598 & 627 & 55.6 \\
\hline - LUCIE+] & 1324 & 1396 & 1396 & a)! & 1541 & 1541 & 611 & $182 B$ & .928 & 1828 & 490 & 17 & 517 & 544 & 569 & 569 & 595 & 674 & 194 & 67 \\
\hline - LUCI & 952 & 1031 & 1031 & 1095 & 117 & 117 & 1243 & 1320 & 1320 & 1387 & 362 & 192 & 392 & 418 & (4) & 447 & 472 & 502 & 502 & 527 \\
\hline MER & 60 & 825 & 890 & 890 & 954 & 017 & 017 & 1020 & 143 & 43 & 336 & 5 & 3 & 393 & 1 & 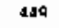 & 19 & 176 & 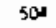 & 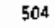 \\
\hline$f(x-1$ & 158 & 214 & 1214 & 1269 & 324 & 1324 & 1481 & 1481 & 1481 & {$[\$ 81$} & 527 & 52 & 552 & 577 & 12 & 502 & 674 & 674 & 74 & 79 \\
\hline$R \mathbf{r}-2$ & 38 & 988 & 043 & 1098 & 098 & 255 & 55 & 1255 & 255 & 255 & 51 & 1 & $\mathrm{rn}$ & 501 & 1 & 72 & 12 & 72 & 8 & 32 \\
\hline WOUEMA & 9036 & 3263 & 3488 & J48B & $371]$ & 3930 & 3930 & 4149 & 4369 & 4369 & 541 & 1 & 21 & 621 & 60 & 699 & 99 & 337 & 76 & 76 \\
\hline 5OUEHAMHA-2 & 2980 & 114 & 3114 & 3339 & 3562 & 3562 & 3780 & 3999 & 3999 & 4219 & 515 & 54 & $\$ 54$ & 594 & ه广 & 634 & 72 & 711 & 11 & 750 \\
\hline RiE HILE & 33 & 33 & 201 & 269 & 269 & 1446 & 446 & 100 & 1446 & A46 & 25 & 5 & 56 & 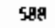 & 18 & 70 & 0 & 670 & 70 & 50 \\
\hline$A N$ & 1165 & 200 & 1252 & 1295 & 1338 & 1380 & 1422 & 1464 & 1657 & 1657 & 537 & 57 & 577 & (5) & 617 & 6.75 & 56 & 7 & 4 & 64 \\
\hline KKEY POINT-3 & $\$ 115$ & 115 & 1174 & 1233 & 1233 & 390 & 390 & 1390 & 390 & 1390 & sog & 509 & 5 & 563 & 53 & 636 & 16 & 636 & 630 & 36 \\
\hline KEY DC & 1216 & 11273 & 1232 & 1232 & 1789 & 1389 & 1389 & : 389 & 1389 & 1389 & 511 & 37 & 564 & 564 & 636 & 635 & $63 \mathrm{~F}$ & 636 & 36 & 36 \\
\hline ANT Y Y & 58 & 341 & 2924 & 3007 & 3375 & 3375 & 375 & 3315 & 3375 & 3375 & 504 & 9 & 534 & Sos & 616 & 615 & 16 & 16 & 516 & $? 6$ \\
\hline G'LE-i & $? 1$ & 379 & 1037 & 2094 & $11 \leqslant 1$ & 1207 & 263 & 1319 & 315 & 1431 & 425 & 1 & 178 & 504 & 11 & $\$ 56$ & 32 & 608 & 34 & 660 \\
\hline$\because L E-2$ & 8 & 756 & 814 & 831 & 928 & 964 & 1040 & 1096 & 1152 & 1208 & $32 z$ & 18 & 375 & 401 & 8 & 454 & 479 & sos & II & 5: \\
\hline ERFORU & 31 & 095 & 160 & 160 & 1225 & 1297 & 352 & 1414 & 1477 & 3477 & 436 & 464 & 891 & 491 & 9 & $54 \$$ & 573 & 5 द्रे & 20 & 625 \\
\hline TS the & 855 & 931 & 1006 & 1006 & 1081 & 1155 & 1155 & 1228 & 1228 & 1302 & 192 & 15 & 44 & 449 & $i$ & 514 & 5) 4 & 545 & if & 570 \\
\hline 5 & 5 & 785 & 381 & 936. & 936 & 1010 & 1063 & 1ปgy & 1156 & 1156 & 150 & 50 & $38 \mathrm{~B}$ & 477 & & 450 & 48? & $₫ 82$ & a & 116 \\
\hline$P-i$ & 639 & 697 & 155 & 912 & 869 & 925 & 99] & 1037 & 1093 & 1149 & 291 & A & 394 & 370 & 16 & 422 & 77 & 473 & 99 & $?$ \\
\hline $8-2$ & & 17 & & 47 & 96 & 42 & 8 & 4 & 181 & 27 & 541 & & $59 \%$ & 62 & & 67 & & 30 & 6 & 83 \\
\hline$f-3$ & 902 & 971 & 1039 & 1107 & 1175 & 1202 & 306 & 1374 & 3400 & 1507 & 285 & 41 & 448 & $47 ?$ & 503 & 532 & 560 & $5 \square$ & 17 & FAF \\
\hline F CAE & 762 & 762 & $82 T$ & 591 & 391 & 955 & 1018 & 1018 & 1081 & 1644 & 2 & & & & & 441 & & $47 n$ & a & 2 \\
\hline KEE & $68 \mathrm{i}$ & SBI & $6+11$ & $B 81$ & 689 & $6 \times 1$ & 68 & 81 & 681 & [B] & 160 & $15 \%$ & 60 & $16 \Gamma^{\circ}$ & 160 & $16 \mathrm{~F}$ & iאn & : אก & חאן & L fin \\
\hline $110 \mathrm{H}$ & $3 \mathrm{~B} 6$ & 386 & 466 & 5 कब & 546 & F⿳士口 & 701 & & 178 & 855 & וזיו & & vd & $? 3 A$ & & 31 & & $3 ! ?$ & f6. & ifrir \\
\hline I. LUV & 06 & 187 & $x$ & 467 & 546 & 546 & 523 & " & 300 & דז & 136 & 17 & [7] & 208 & $73 \mathrm{R}$ & 238 & 27,1 & 303 & . & '] \\
\hline$i M-i$ & 1352 & 395 & 34 & 81 & 24 & 11 & 17 & 1717 & 17 & 1717 & $S 1 R$ & 59 & 57 & 677 & 697 & 85 & 5 & 95 & aद & ئ \\
\hline$N-2$ & $12 \mathrm{\forall} 2$ & 1326 & 1369 & 1412 & 1455 & 1648 & 1648 & $164 \mathrm{~B}$ & 1648 & JKAB & 586 & S106 & 524 & 654 & $5,6.5$ & 753 & 753 & 153 & 153 & 753 \\
\hline 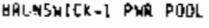 & 166 & 150 & 160 & 160 & 16 & 160 & 160 & 1 & 160 & 160 & $7 !$ & $?$ & $\because$ & 1 & !! & 11 & 7 & $\because$ & 1 & 11 \\
\hline IUASSIC1CK-2 PLR PUUL & {$[44$} & 104 & 144 & 14de & 144 & 1sa & 144 & 1 & 144 & 144 & 6 & I & 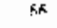 & hat & in & 6 & 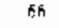 & if & is & s. \\
\hline KIS-d d & 153 & 753 & 153 & 753 & 153 & 353 & 75 & 75. & 153 & 753 & 145 & 10 & 145 & 349 & 145 & :45 & 145 & 105 & 145 & 145 \\
\hline HRP [5-P & 459 & 459 & $45 y$ & a59 & 459 & 459 & 459 & 459 & 459 & 499 & $: 77$ & 177 & $1 m$ & 133 & $! ? 7$ & 177 & 17 & 177 & 177 & $19 ?$ \\
\hline ST YALLEY - BWK & AlE & 4 & 418 & 4it & 41 & 418 & 4. & 4 & AlB & 41 & 66 & of & 66 & fin & 66 & 66 & $6 \overline{\mathrm{i}}$ & 56 & 6 & 66 \\
\hline it & 235 & 235 & 207 & 233 & 295 & 235 & 207 & 233 & 235 & 233 & 7 & 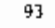 & 93 & 93 & 73 & 93 & 93 & 93 & 9? & $\omega$ \\
\hline a & 2216 & 3412 & 4663 & 7016 & 9236 & 345 & 18114 & 95 & 75 & 83 & 419 & 649 & 877 & 321 & 729 & 2310 & 3013 & 030 & 824 & 735 \\
\hline ster & 802 & 14 & 268 & 18 & 85 & 16 & 25 & 10286 & 334 & 67 & 370 & j & 035 & 16) & 218 & 353 & 53 & 1591 & 9946 & 230 \\
\hline
\end{tabular}


TABLE A.4. (contd)

\begin{tabular}{|c|c|c|c|c|c|c|c|c|c|c|c|c|c|c|}
\hline \multirow[b]{2}{*}{ ACTOQ } & \multicolumn{7}{|c|}{ ASSEEMBLIE } & \multicolumn{7}{|c|}{ MT J IMM } \\
\hline & 015 & 015 & 16 & & 018 & 195 & 2020 & श्रा & 201 & OI & 2017 & 2018 & 3రाढ़ & \\
\hline XANSAS MUCL OME-1 & $\lceil 524$ & $\$ 524$ & 1524 & 1524 & 1524 & 1524 & 1524 & 706 & 706 & 706 & 706 & 706 & 706 & \\
\hline ARKANSAS MUCL ONE- & 1361 & 1361 & 1361 & 1361 & 1361 & $136 !$ & 1361 & $\sin$ & 580 & 580 & 580 & 580 & 580 & \\
\hline BEAYER YALLEY-i & 1471 & 1471 & 2471 & 1471 & 2471 & 1471 & 1471 & 679 & 679 & 679 & 679 & 679 & 679 & \\
\hline BEAVER YALLEY-Z & 1089 & 1153 & 3217 & 1257 & 1281 & 1345 & 1345 & 503 & 532 & 562 & 562 & $\$ 91$ & ร2ป & \\
\hline BE1LEFONTE-1 & 1315 & 1399 & 1399 & 1484 & 1484 & 1569 & 1654 & 579 & 616 & 616 & 653 & 653 & 691 & \\
\hline BELLEFONTE-2 & 1168 & 1188 & 1252 & 1252 & 1337 & 1422 & 2422 & 516 & $\$ 16$ & 553 & 553 & 59자 & $\$ 28$ & \\
\hline BIG ROCK DOINT & 504 & 504 & 500 & 504 & sod & 504 & 504 & 65 & 65 & 65 & 65 & 65 & 65 & \\
\hline BRAIDWOOD-i & 1397 & 1447 & 1497 & 1547 & 1598 & 1649 & 1700 & 591 & 612 & 633 & 654 & 676 & 897 & \\
\hline BRAIDWODO-2 & 1269 & 1319 & 1369 & 1420 & 1471 & 1522 & 1573 & 537 & 558 & 579 & \$N30 & 622 & 644 & \\
\hline BRDWNS FERAY-1 & 4861 & 4861 & 4961 & 4861 & $486 !$ & $A B 6]$ & 9.861 & 960 & 860 & 860 & 860 & 960 & 860 & \\
\hline HROWNS FERRY-2 & 5072 & 3072 & 5072 & Sol2 & 5072 & 5072 & 5012 & 895 & 895 & 395 & R95 & 395 & 995 & \\
\hline GROWNS FERRY-3 & 4932 & 4932 & 4932 & 4932 & 4932 & 4932 & 4932 & 875 & H75 & 875 & 875 & 875 & 815 & \\
\hline ERUNSW [CX -1$]$ & 4093 & 4093 & 4093 & 4093 & 4093 & 4093 & 4093 & 765 & 765 & 165 & 765 & 765 & 765 & \\
\hline BRUNSHICK-Z & $3 \Varangle 62$ & 3962 & 3862 & 3862 & 1862 & 2862 & 3962 & 122 & 322 & 122 & 722 & 722 & 722 & \\
\hline [RUNM-1 & $! 443$ & 1493 & 1543 & 1593 & 1644 & 1695 & 1746 & 610 & 631 & 652 & 674 & 95 & 717 & \\
\hline ROH-2 & 1404 & {$[454$} & 1504 & 1554 & 1605 & 1656 & 1707 & 594 & 615 & 636 & 657 & 879 & 300 & \\
\hline LLAWAY -1 & 176 & 1553 & 1631 & 1631 & 1709 & 1787 & 1787 & 631 & 663 & 696 & 696 & 729 & 162 & \\
\hline HLYERT CLIFFS-1 & 1794 & 1794 & $17 y d$ & 1794 & 1794 & 1794 & 1794 & 693 & 693 & 693 & 693 & 593 & 693 & \\
\hline LYERT CLIFFS-2 & 1698 & 1694 & 1698 & 1699 & 1698 & 1698 & 1698 & 657 & 657 & 657 & 657 & 557 & 65) & \\
\hline TAWBA-! & 1366 & 1419 & 1074 & 1528 & 1582 & 1582 & 1636 & 579 & fio & 624 & 647 & 670 & $67 n$ & \\
\hline TAMBA-2 & 1281 & 1336 & 1336 & 1390 & 1445 & 1499 & 1553 & SA3 & 566 & 565 & 589 & 512 & 635 & \\
\hline [NTON-1 & 3685 & 3685 & 38.85 & 4096 & 4046 & 4287 & S489 & 674 & STA & 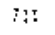 & 747 & ta? & IASI & \\
\hline WAICHE PEAK-1 & 1629 & 1685 & 1742 & 1799 & 1856 & 1913 & 1970 & $67 t$ & 593 & 716 & 739 & 562 & 795 & \\
\hline MANGHE PEAK -2 & sa & 1611 & 1668 & 1725 & t $79 ?$ & 1839 & $1 B 9 h$ & 539 & 662 & 685 & ing & 731 & 754 & \\
\hline IKNECTICLST $\mathrm{Y}$ & 1404 & 1404 & 1404 & 1404 & $: 404$ & 1404 & 1 snde & $5+\pi$ & 576 & 576 & 576 & $5 i k$ & 576 & \\
\hline OPEA & I8 & 3858 & 3958 & 3858 & 3958 & 3858 & 3ese & $? 1$ & 911 & $: ! 1$ & 311 & 11 & $? 3:$ & \\
\hline YSTAL RI & 53 & 1353 & 1353 & 1353 & 1353 & 1353 & 1353 & 628 & $42 R$ & fi2a & $62 R$ & 674 & $\mathrm{fi} 2 \mathrm{H}$ & \\
\hline $10 x-1$ & SA96 & 1896 & 1996 & 1896 & la96 & 1996 & 1996 & 257 & 5) & 457 & RS: & s? & म<? & \\
\hline $0 \mathbf{k}=?$ & 16 & 1366 & te66 & 1866 & 1856 & 1866 & Jufit & $77 \hbar$ & $19 k$ & $? T K$ & 736 & 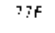 & $T^{\prime} \bar{i}$ & \\
\hline $415-B E S S E-\frac{1}{2}$ & 254 & 2250 & 1254 & 125.4 & 1254 & $17 \leqslant 4$ & 1254 & 588 & 568 & Sext & SмH & بربّi & ??? & \\
\hline ABLO CANYGN-1 & 357 & 1423 & 1460 & 1536 & 1597 & 1694 & 1649 & 028 & 6.3 & $5 ?\}$ & : $n \leqq$ & 31 & $i 4 \hat{i}$ & \\
\hline AHLV CAMY & $|28|$ & $: 336$ & 1394 & :449 & 15.36 & 1506 & !\$63 & 588 & 613 & 540 & 665 & 64] & 691 & \\
\hline ESOEN-1 & id 3 & 683 & 683 & 683 & 663 & 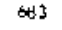 & 3 & 69 & 9 & 69 & 59 & 39 & 89 & \\
\hline DEM-2 & 546 & Sbat & 4546 & 45.46 & 4546 & 4546 & ASAG & 322 & 822 & 322 & 872 & a22 & म27 & \\
\hline ESDEN -3 & 8020 & \$ 120 & 4020 & ง ڤีڤ & 4020 & 9020 & 4020 & 847 & :97 & ist & 34 & 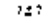 & $\Delta 7$ & \\
\hline ANE ARMOLD & 160 & 2860 & 2860 & 2860 & 2860 & $2 \mathrm{H}^{\circ} \mathrm{O}$ & 2460 & 525 & 525 & 525 & 525 & 525 & 525 & \\
\hline$R \operatorname{LE}\{-]$ & $: 650$ & 1650 & 1650 & 1650 & 1650 & $1650^{\circ}$ & 1650 & 761 & 761 & 151 & 361 & 361 & 76: & \\
\hline LRLÉY $=2$ & 1499 & $149 y$ & 1499 & 1499 & 1499 & 1499 & 1498 & 689 & 689 & 589 & 684 & 644 & 680 & \\
\hline 4] -2 & tods & 4337 & 4333 & 4587 & 4587 & 4A4 & $2 \times d 4$ & 143 & 389 & 789 & 834 & 894 & क्से & \\
\hline TZPATRICX & 4572 & 4572 & 4572 & 4532 & 4572 & 4572 & $₫ \leqslant \geqslant$ & $\times 3:$ & +33 & 837 & $9 ?$ & p1? & H? & \\
\hline CAEROUNK- & $: 069$ & 1069 & 1069 & 1069 & 1069 & 1069 & 1069 & 3id & 3ns & $x e 4$ & $3,4.4$ & و & ד & \\
\hline Wha & 10006 & 1006 & 1006 & 1006 & 1006 & 1006 & 1006 & 366 & $26 k$ & $36 \AA$ & $36 \mathrm{~F}$ & ?.6F & $36 \pi$ & \\
\hline ANo GULF-l & 4423 & 4653 & $48 B 4$ & AHBA & 5116 & 53.49 & 5340 & 179 & $\$ 20$ & 960 & $86 n$ & $8 ח 1$ & $9 s ?$ & \\
\hline 110 GULF- & 23 & 411 & 4900 & 5080 & 5290 & 5471 & 5662 & A31 & $\rho_{6 G 5}$ & 9001 & 935 & $\$ 70$ & $: \cap 05$ & \\
\hline RRIS $=1$ & 1024 & $102 a$ & 1070 & 1116 & 1162 & 1208 & JZNA & 471 & 47) & $\$ 92$ & 513 & 535 & $55 \%$ & \\
\hline VILLE & 3074 & 3294 & 3515 & 3515 & $373 t$ & 3959 & 3950 & 563 & 601 & 5.43 & 647 & 84 & 124 & \\
\hline rite & & 2847 & $2 B$ & 3068 & 3290 & 3290 & 3513 & \&R? & $b 2 z$ & 522 & 562 & 0? & $6 \cap 2$ & \\
\hline $1+-1$ & 16 & 186 & 36 & 4386 & 86 & 86 & Hh & 02 & 02 & 02 & 02 & 02 & BD? & \\
\hline
\end{tabular}




\section{TABLE A.4. (contd)}

\begin{tabular}{|c|c|c|c|c|c|c|c|c|c|c|c|c|c|c|}
\hline \multirow[b]{2}{*}{ REACTOR } & \multicolumn{7}{|c|}{ ASSEIMBL, IES } & \multicolumn{7}{|c|}{ MF INM } \\
\hline & $\underline{14}$ & 15 & 16 & 2017 & 201요 & 2019 & 20 & 2014 & $\underline{2015}$ & $\underline{2016}$ & $\underline{2017}$ & $\underline{2018}$ & 2019 & 20 \\
\hline $\mathrm{TCH}-2$ & 644 & 4644 & 4644 & 2644 & 4644 & $464 d$ & 4644 & $\operatorname{Bat} 6$ & 846 & 846 & 846 & 846 & 348 & 246 \\
\hline PE CREEK+1 & 425 & 4088 & 4406 & $4 / 27$ & 4967 & 4967 & 5207 & 774 & 817 & $B 17$ & 960 & 904 & 304 & 948 \\
\hline JMOLDT BAY & 434 & 434 & 434 & 434 & 434 & 434 & 434 & 31 & 31 & 31 & 31 & 31 & 31 & 31 \\
\hline MOIAN pOLNT-I & 160 & 160 & 160 & 160 & 160 & 160 & 180 & 31 & 31 & 31 & $3 \mathbf{l}$ & 31 & $3 \mathbf{i}$ & 31 \\
\hline$A M I A M P Q C M T-2$ & 1524 & 1524 & 1524 & 1524 & 1524 & 1524 & 1524 & 693 & 693 & 693 & 693 & 691 & 693 & 693 \\
\hline INBIAN $90[M T-3$ & 1428 & 1428 & 1428 & 1428 & 1428 & $142 B$ & 1428 & 651 & 651 & 651 & 651 & 551 & 651 & 651 \\
\hline EHALUEEE & 126: & 1260 & 2268 & 1268 & 1268 & 1268 & 1269 & 499 & 39 & 489 & LAS & 49 & 489 & 489 \\
\hline CROSSE & 117 & 117 & 117 & 117 & 117 & 717 & 717 & 90 & 80 & 80 & 80 & 80 & so & 80 \\
\hline SALEE-1 & 3943 & 4139 & 4139 & 4336 & 4533 & 4533 & 4731 & 119 & 754 & 754 & 790 & 825 & 1826 & 962 \\
\hline SALLE-2 & 3942 & 4138 & 4178 & 4335 & 4532 & 4532 & 1730 & 719 & 754 & 754 & 190 & 926 & 826 & 852 \\
\hline [MERICR-] & 156 & $43 / 6$ & 4597 & 4597 & 4919 & sos 1 & 5041 & 160 & 200 & $B \boldsymbol{B O}$ & 840 & 390 & 921 & 921 \\
\hline $\ln R \backslash C X-2$ & 3095 & 3312 & 3312 & 3533 & 3755 & 3755 & 3978 & 563 & 600 & 603 & 643 & 683 & 3 & 324 \\
\hline INE YAAIKEE & 1960 & 1960 & 1960 & 1960 & 1960 & 1960 & 1960 & 37 & 137 & 737 & 137 & 137 & 737 & 737 \\
\hline GUIRE-1 & 1692 & 1692 & 1692 & 1692 & 1692 & 1692 & 1692 & 124 & 724 & 224 & $12 \mathrm{a}$ & 124 & 24 & 724 \\
\hline GUIRE-2 & 6009 & 1600 & 1809 & 1609 & 1609 & 1609 & 1609 & 688 & 698 & $s e 8$ & 688 & 80 & 68 & 698 \\
\hline DLAMO=1 & 961 & 861 & 917 & 974 & 74 & 1031 & 31 & 1 & $\mathbf{I}$ & 427 & 153 & 453 & 480 & 480 \\
\hline THLAMD-2 & 916 & 972 & $\$ 72$ & 1029 & 1086 & 1086 & 1143 & 426 & 52 & 452 & 479 & 506 & 506 & 532 \\
\hline LLSTONE-1 & 4020 & 20 & $\$ 020$ & 1020 & 4020 & 020 & 020 & 124 & 724 & 724 & 724 & 124 & 124 & 724 \\
\hline LLSTONE - 2 & เa99 & 1894 & เ899 & 1699 & Id99 & 1899 & 1899 & 759 & 759 & 159 & 159 & 759 & 59 & 759 \\
\hline LLSTONE-3 & $3 / 4$ & 140 & 1430 & 1487 & 1540 & 1601 & 1659 & 634 & 660 & 660 & 686 & 113 & 3 & 765 \\
\hline JKTICELLO & 3467 & 3467 & 3467 & 3467 & 3487 & 3467 & 3467 & 5 & 5 & 25 & 35 & 25 & 25 & 25 \\
\hline INE MILE POIM & 3504 & 3504 & 3504 & 3504 & 3504 & 3504 & 3504 & 34 & 14 & 534 & 6.34 & 34 & 34 & 534 \\
\hline INE AILE POLL & 3SSI & 3551 & 3786 & 3786 & 4022 & 4258 & a & 10 & 0 & 13 & 683 & 15 & 7 & 57 \\
\hline WRTH $A N N A=1$ & 1510 & 1510 & 1520 & 1510 & 1510 & Istu & 1510 & 695 & 95 & 695 & 695 & 695 & 695 & 695 \\
\hline WWTH AWNA-2 & 2429 & 1429 & 1429 & 1429 & 1424 & 1429 & 9 & 657 & 657 & 657 & 557 & 653 & 657 & 657 \\
\hline CUNEE - 1 & 1718 & $1 / 18$ & 1716 & 1t18 & 1718 & 1718 & 1718 & 796 & 198 & 198 & 298 & 198 & 798 & 798 \\
\hline EUMEE - 2 & 225 & 1425 & 2425 & 1425 & 1025 & 1425 & 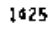 & 861 & 681 & i & $f_{61}$ & 61 & 16 & 61 \\
\hline CONEE- $]$ & LI5d & 1158 & 1158 & $115 d$ & 1154 & 1158 & 1158 & 537 & 31 & 537 & 537 & 37 & 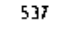 & 537 \\
\hline STER LH & 65 & 3665 & 3665 & 3665 & 1665 & 3665 & 16 & 5 & & 2 & 555 & 655 & 155 & 655 \\
\hline LL ISADES & 1592 & 1592 & 1592 & 1592 & 1592 & 1592 & 1592 & 627 & 1 & 7 & 527 & 27 & 7 & 27 \\
\hline LO VEROE-I & 549 & 1706 & 1753 & 1820 & 1878 & 1936 & 19 & {$[1]$} & 8 & 763 & {$[R 7$} & 813 & 338 & 863 \\
\hline ALO NEROE-? & 1595 & 1652 & 1700 & 1166 & 1824 & เg42 & 1900 & 689 & 14 & 9 & 154 & 789 & 14 & 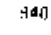 \\
\hline LLO YEROE-3 & 158 & 1637 & 1694 & 3752 & 1810 & เу68 & 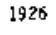 & 83 & กB & 33 & $159+$ & $? 84$ & 909 & d3d \\
\hline EACH $30 \mathrm{Tr}_{\mathrm{r}} \mathrm{M}-2$ & 5634 & 5634 & 5634 & 5634 & 5.634 & 5634 & 5634 & 1026 & 1026 & $2 \pi 26$ & 1026 & 1026 & :026 & 1925 \\
\hline EACH BUT?ZM-7 & 3632 & $\$ 632$ & $\$ 632$ & 5632 & $\$ 632$ & 5632 & 5632 & 1025 & 1025 & 1025 & 1025 & 1025 & 1025 & 1025 \\
\hline ARY-1 & 41 & 4906 & 5167 & 5167 & $542 B$ & 5689 & 5649 & $\$ 48$ & 5 & 12 & 942 & 990 & 1038 & 1020 \\
\hline EHHY 2 & $3(y)$ ? & 4097 & 4356 & 4617 & 4617 & $4 y 78$ & 5140 & 143 & 743 & 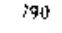 & 338 & 818 & 385 & 933 \\
\hline I L Lا [N- & 3805 & 3405 & 3805 & 3905 & 2805 & 3805 & ] 805 & संबБ & $6 d 6$ & 68 & 586 & GRG & 36 & 686 \\
\hline UINT GEACH-1 & 1206 & IIv6 & 1106 & โเปซ & 1106 & $! 106$ & 1106 & 407 & 407 & भी? & 407 & 407 & $\Delta 0 t$ & मी \\
\hline ULVT $\triangle E A \mathrm{CH}-2$ & 2094 & $10 y 4$ & 2094 & เย94 & 1094 & 1094 & 1094 & 404 & 404 & 404 & 404 & 904 & 104 & 31\%8 \\
\hline RALIHIE IST,ANIU-1 & 1301 & 1301 & 1311 & 1301 & 1301 & 1301 & 1301 & 520 & 520 & 320 & 520 & $\$ 20$ & 520 & 320 \\
\hline DKALKTE :SLAAIU-2 & 1262 & 1262 & 1262 & 1262 & 1262 & $: 262$ & 1262 & 505 & 205 & 505 & 5.35 & 05 & 505 & 305 \\
\hline :LAU LITIES-1 & 4314 & 4314 & 4314 & 5314 & 4314 & 4314 & 4314 & 778 & 774 & $n 4$ & 174 & 174 & 874 & $1 ? 4$ \\
\hline LAD CIT:ES-2 & 4077 & 4017 & 4077 & 4077 & 4077 & $40 n$ & 4077 & 134 & 134 & 334 & 134 & 134 & Aגו & ija \\
\hline Kanclios & 1442 & 1442 & 1442 & 2442 & 1442 & 1442 & 1442 & 669 & 669 & 569 & 669 & 569 & 669 & 569 \\
\hline [XEA DEMD-I & $3 / 64$ & 3922 & 4059 & 4193 & 4327 & 4461 & +594 & 613 & 397 & 720 & $1 \% 4$ & $16 \mathrm{~B}$ & 792 & 816 \\
\hline 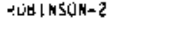 & 163 & 1163 & 1163 & 1163 & 1163 & 1163 & 1163 & 500 & 500 & 500 & 510 & 500 & 500 & 500 \\
\hline & 82 & 82 & 192 & Aa & 82 & 82 & 82 & 130 & 30 & 330 & ?30 & ח3י & ? 3n & 330 \\
\hline
\end{tabular}


TABLE A.4. (contd)

\begin{tabular}{|c|c|c|c|c|c|c|c|c|c|c|c|c|c|c|}
\hline \multirow[b]{2}{*}{ QENCTOR } & \multicolumn{7}{|c|}{ A5SERMLLIES } & \multicolumn{7}{|c|}{ MTI IM } \\
\hline & 2014 & 2015 & 2므16 & 2017 & 2018 & 0.19 & 2020 & 2014 & 2015 & 016 & 2917 & 2018 & 19 & 20 \\
\hline$L \subseteq M+2$ & 1528 & 1529 & 1528 & 1528 & 1529 & 1528 & 1528 & 133 & I3 & 133 & 13 & 733 & 133 & 33 \\
\hline 5AN ONOFRE-1 & 08 & 108 & 308 & 709 & 708 & 108 & 708 & 262 & 262 & 262 & 252 & 52 & 262 & 262 \\
\hline SHM ONOF RE-2 & 1341 & 1341 & 1341 & 1341 & 1341 & 1341 & 1341 & 561 & $\$ 61$ & 561 & 561 & la & 61 & $6 i$ \\
\hline SAN OMOFRE-3 & 1121 & 1191 & $115 !$ & 1262 & 1262 & 1333 & 1404 & 469 & 498 & $\$ 90$ & 529 & 23 & 58 & 598 \\
\hline SEABROOK - 1 & 1542 & 1598 & 1655 & 1312 & 1769 & 1926 & 1983 & 653 & 677 & oi & 125 & 19 & 73 & 797 \\
\hline SEABROOAT-2 & 1213 & 1269 & 1325 & 1382 & 1439 & 1496 & 1553 & 514 & 7 & 561 & 585 & 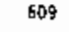 & 3 & 88 \\
\hline SEOUDYAH-1 & 1595 & 1595 & 1595 & 1595 & 1595 & 1595 & 1595 & 204 & 704 & 104 & 04 & 94 & 04 & 04 \\
\hline SEQUDYAH-Z & 1513 & 1513 & 1513 & 1513 & 13 & 3 & {$[513$} & 673 & 673 & 673 & 673 & 673 & 3 & 73 \\
\hline SHOREHNM & $293 ?$ & 3094 & 3252 & 3252 & 11 & 5.70 & 3570 & 539 & 567 & 596 & 596 & a & 15 & $\$$ \\
\hline SONTH TEXIS PAQN=1 & 1445 & 1499 & 1554 & 1609 & 1664 & 1719 & 1774 & 778 & $8 d 7$ & 837 & 966 & 10 & 45 & 55 \\
\hline 5OUTH TEXAS AOU-2 & 1273 & 1323 & 1382 & 1437 & 12 & 7 & 1602 & $\$ 5$ & 4 & 4 & 4 & & 3 & 2 \\
\hline 5T. LUCIE-1 & 1828 & 1828 & 1829 & 1828 & 1928 & 1828 & 1828 & 674 & 674 & 674 & 674 & & 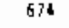 & 3 \\
\hline 5T. LUCTE-? & 1464 & 1464 & 1531 & 1609 & & 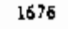 & 17 & 6 & 6 & 92 & 2 & & 3 & \\
\hline SLTTER & 300 & 1300 & 2300 & 1300 & 1300 & 1300 & 1300 & $\$ 73$ & 573 & 573 & 573 & 3 & 3 & 3 \\
\hline SLPRY - I & 81 & 81 & 181 & I & & 1 & 14 & 4 & & 74 & 4 & & 14 & 4 \\
\hline SURRY -2 & 255 & 1255 & 1255 & 1255 & 1255 & 1255 & 1255 & 572 & 512 & 572 & $3 / 2$ & 2 & 12 & ? \\
\hline SUSQUEHAMMK-1 & 588 & 4808 & 4800 & s029 & 5251 & 3251 & 30 & 815 & 844 & 854 & 93 & & 32 & 1 \\
\hline 5USONEHANKA-2 & 9438 & 4438 & 4.659 & 4891 & 4881 & 5103 & 5326 & 138 & 780 & 827 & 666 & 66 & 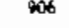 & \\
\hline ?HREE MILE ISLAMD-1 & 1446 & 1446 & 1446 & 1446 & 14 & 116 & 14 & 670 & 670 & 670 & 70 & & v & 0 \\
\hline TROD AN & 1651 & 1657 & 1657 & 165? & 657 & 1657 & 1657 & 361 & 64 & 364 & 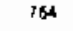 & & 54 & 4 \\
\hline TURKEY MDINT - - & 1390 & 1390 & 1390 & 1390 & 139 & 1390 & [3 & 636 & 636 & 636 & 16 & & 36 & 6 \\
\hline SUAKEY DOINT-4 & 899 & 1399 & 1399 & 39 & 1389 & 99 & 13 & 636 & 6 & 36 & 6 & & 36 & 6 \\
\hline YERMONT YKMKEE & 15 & 3375 & 3375 & 75 & & & & 616 & & 16 & 6 & & & \\
\hline YOGTLE-1 & 148? & 1543 & 1599 & 1656 & 173 & 10 & 1827 & 685 & 111 & 137 & 101 & & 8 & \\
\hline YOGTLE-2 & 1264 & 1320 & 1373 & 1434 & 1491 & 1548 & 1605 & 583 & $\infty \mathbf{0}$ & 635 & 861 & & 14 & \\
\hline WATERF ORO-3 & 41 & 1603 & 1669 & 1731 & 1195 & 5 & 186 & 553 & 680 & 707 & 34 & & 51 & \\
\hline WATTS DAR - 1 & 1375 & 1375 & 1449 & 1523 & 1523 & 1597 & $159\}$ & 612 & $\$ 12$ & $6+5$ & 578 & 9 & 10 & 10 \\
\hline WATTS BAR-Z & 1229 & 130 & 1303 & 13 & 1451 & 14 & 15 & 547 & 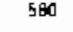 & 580 & 613 & & & \\
\hline WHP-i & 1205 & 1261 & 1317 & 1374 & 1431 & 1469 & 1545 & 550 & $\$ 75$ & 601 & 627 & 3 & 9 & 05 \\
\hline WHP -2 & 673 & 4620 & 4768 & 4916 & 5064 & & 53 & 809 & & 863 & $\$ 0$ & & & \\
\hline WNP - 3 & 1573 & 1640 & 1707 & $1 m$ & $18 \alpha 1$ & 1908 & 1976 & 674 & 203 & 732 & 761 & $\infty$ & 19 & 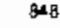 \\
\hline WOAF CAEETK & 1144 & 1207 & 1211 & 1271 & 1315 & 1399 & 1399 & 528 & 557 & 586 & 586 & 6 & 65 & 5 \\
\hline YANKEE & 681 & 581 & 681 & 681 & 681 & 681 & 691 & $1 \infty 0$ & 160 & 160 & 160 & 160 & 160 & 160 \\
\hline YELLON CAEEK-I & 855 & 932 & 1010 & 1010 & 100 & 1166 & 1168 & 368 & 401 & 434 & 334 & 467 & 00 & 00 \\
\hline TLLLOW CREEK-2 & 85 & $95 \mathbf{s}$ & 932 & 1010 & 1010 & 1008 & 1166 & 388 & 368 & 401 & 434 & 434 & 467 & $\$ 00$ \\
\hline $210 n-1$ & $1 !$ & 171 & 1717 & 1117 & 171 & 1717 & 1717 & 785 & 195 & 385 & 795 & 785 & 585 & 795 \\
\hline $210 \mathrm{M}-2$ & 1648 & 1648 & 1648 & 1649 & 1648 & $164 \mathrm{~B}$ & 1648 & 753 & 353 & 753 & 153 & 153 & 753 & 753 \\
\hline BRUNSWICK-1 DHR POOL & 160 & 160 & 160 & 160 & 160 & 160 & 160 & 7 & 11 & 11 & 71 & 11 & 11 & 1 \\
\hline 9RUMSWICK-2 PAO POO & 144 & 144 & 144 & 14 & 14 & t\$4 & $1 \$ 4$ & 65 & 86 & 66 & 66 & 66 & 66 & 66 \\
\hline MOAR IS - 6WR & 153 & 753 & 753 & 153 & 753 & 353 & 353 & 145 & 1 As & 145 & 145 & 195 & 145 & 145 \\
\hline MOAR IS-PWR & 159 & 459 & 850 & 459 & 459 & 459 & 459 & 17 & $m$ & 171 & 27 & 177 & 177 & $17:$ \\
\hline HEST YALLEY-BKR & 418 & 418 & $\$ 18$ & 418 & 418 & 418 & \$18 & 66 & 66 & 66 & 56 & 66 & 66 & 56 \\
\hline WEST YALLEY - PWR & 235 & 235 & 235 & 235 & 235 & 235 & 235 & 93 & 93 & 93 & 9] & 93 & 93 & ב⿱\zh18灬 \\
\hline GWR GSUERIC & 36979 & 41530 & $\$ 7790$ & 54108 & 59780 & 65870 & 73387 & 6896 & $n 51$ & 8687 & 10041 & 1079 & 12190 & 13565 \\
\hline PWA GENERIC & 19581 & 23437 & 27048 & 31194 & 35459 & 39627 & 44133 & 8671 & 10337 & .0493 & 12280 & 14101 & 15386 & 17808 \\
\hline
\end{tabular}




\section{TABLE A.4. (contd)}

tores

\begin{tabular}{|c|c|c|c|c|c|c|c|c|c|c|c|}
\hline & 1983. & 1984 & 1985 & 19.165 & 1987 & 1988 & $: 989$ & $i 990$ & 1991 & 1992 & 1993 \\
\hline PLQ ASSEMBL, & 14114 & 15744 & 18054 & 20085 & 22611 & 25663 & 29755 & 31951 & 35163 & 28120 & 42314 \\
\hline Pur me IhW & 5953 & C65: & 1663 & 8539 & 9638 & 10954 & 12325 & 63705 & 15003 & 16840 & 18209 \\
\hline 口U ASSEmaLY & 23157 & 25574 & 29105 & 31707 & 35200 & 39310 & 43428 & 47703 & 52467 & 56765 & 61629 \\
\hline ם & sids & 4605 & 5058 & 5705 & 6333 & 7076 & 7819 & Bsas & 9443 & 10217 & 11095 \\
\hline TOTAL ASSEMALY & 37271 & 41318 & A6:59 & 51792 & $\$ 7817$ & 64973 & 72193 & 79654 & 37630 & 95465 & 103943 \\
\hline \multirow[t]{2}{*}{ TOTAL MTHIM } & 10142 & 11267 & 12722 & 14244 & 15912 & 18030 & 20145 & 22290 & 24537 & 26857 & 2304 \\
\hline & 1004 & 1995 & 1996. & 1997 & $199 \mathrm{~B}$ & i999 & 2000 & 2001 & 2002 & 2003 & \\
\hline PWR ASSEIELT & 46146 & 49822 & 53653 & 57701 & 61436 & 65479 & 69434 & 13247 & 7190 & 81729 & \\
\hline Sid MTLH & 19872 & 21476 & 23140 & 24903 & 26526 & 28291 & 30015 & 31692 & 33512 & 35399 & \\
\hline 3HR ASSEMLYY & 56593 & 71643 & nog8 & 32.283 & 87795 & 92035 & 99079 & $10395 a$ & 120415 & 11638: & \\
\hline Que NTIF & 12005 & 12900 & 17880 & 14835 & 15810 & 16756 & 17834 & 19700 & 19884 & 20972 & \\
\hline POTAL ASSERTLY & 112839 & 121465 & 130751 & 140084 & 149231 & 1585514 & 168473 & 177)0s & $1 B 7626$ & 298110 & \\
\hline \multirow[t]{2}{*}{ roture netion } & 38877 & 34376 & 37020 & 39737 & A2336 & 45046 & 47849 & 50395 & 53396 & 56371 & \\
\hline & 2004 & 2005 & 2906 & 2007 & 2008 & 2000 & 2010 & 2011 & 2012 & 2013 & \\
\hline PLA ASSEMALY & 48139 & 90916 & 95498 & 100418 & 105022 & 112791 & 118905 & 124328 & 130362 & 135773 & \\
\hline PWh mTthm & 37341 & 39421 & 41474 & 43593 & 46036 & 49006 & 51693 & 54061 & 56749 & $\$ 125$ & \\
\hline UW ASSERIT & 122459 & $1299 / 9$ & 136444 & 145834 & 154611 & 164896 & 173174 & $1 \theta נ 37]$ & 191105 & 198803 & \\
\hline SWR NTIHW & 22070 & 23429 & 24604 & 26335 & 27902 & 2979 & 31299 & 33197 & 34615 & 26031 & \\
\hline TOTAL ASSEMBLY & 2005597 & 220895 & 232032 & 246359 & 260633 & 277687 & 292074 & 307705 & 321467 & 334576 & \\
\hline \multirow[t]{2}{*}{ DOTA RTIHA } & 59411 & 22850 & 68078 & 69929 & 13937 & 78785 & $829 \$ 2$ & 87258 & 91363 & 95156 & \\
\hline & 2014 & 2015 & 2016 & 2017 & 2018 & 2019 & -2020 & & & & \\
\hline PUR ASSEMBLY & 141846 & {$[47543$} & 153022 & 159076 & 165184 & 174321 & $177 s 13$ & & & & \\
\hline 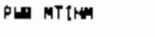 & 61774 & 64255 & 66633 & 69261 & 11900 & 74533 & 11213 & & & & \\
\hline HUa ASSEMGLY & 208009 & 215754 & 224395 & 233415 & 242191 & 251031 & 261018 & & & & \\
\hline BE MTLM & 37318 & 39135 & 40719 & 42361 & 43965 & 45571 & 47392 & & & & \\
\hline TQTAL NSESLIY & 349855 & 363297 & 37747 & 392491 & 407375 & 422352 & 438531 & & & & \\
\hline TQTAL NTIF & 99493 & 103389 & 107752 & 111622 & 115861 & 120123 & 124605 & & & & \\
\hline
\end{tabular}




\section{TABLE A.5. Middle Case Maximum At-Reactor Capacity - Projected Annual Storage Requirements}

\begin{tabular}{|c|c|c|c|c|c|c|c|c|c|c|c|c|c|c|c|c|c|c|c|c|c|c|c|c|}
\hline & & & & & & ASSER & & & & & & & & & & & & & MTIKM & & & & & \\
\hline$P O Q$ & I\$55 & T967 & T58\% & T\$क9 & I0;0 & I911 & I I9y2 & 7539 & IV90 & I595 & $1996-$ & Ig9r & T1965 & Ig: & Igger & Ty8\% & 1990 & 19912 & $\underline{192}$ & Ty9g & 199? & $\underline{1995}$ & T396 & 1997 \\
\hline MILL,5TOHE-2 & 50 & 59 & 9 & & 70 & So & 65 & & 65 & 65 & $\omega$ & & 20 & 24 & 28 & & 28 & 2) & 26 & & 26 & 26 & 26 & 0 \\
\hline DM ISNOE 5 & & 2 & 59 & & 60 & 59 & & 60 & 60 & & 61 & 52 & & 1 & 23 & & 23 & 23 & & 23 & 23 & & 24 & 24 \\
\hline TURKEY POTXT-4 & & 6 & 55 & & 55 & 57 & & 55 & 58 & & 58 & $\infty$ & & 3 & 25 & & 25 & 26 & & 25 & 21 & & 26 & 28 \\
\hline $5 T$. LUCIE -1 & & 3 & & 12 & 70 & & 73 & $n$ & & 14 & 72 & & & 1 & & 28 & 26 & & 27 & 26 & & 27 & 26 & 0 \\
\hline MILLSTOHE-1 & & 49 & & 172 & & 173 & & 176 & 175 & & $\operatorname{tgd}$ & & & 9 & & 30 & & 31 & & 31 & $3 I$ & & 32 & 2 \\
\hline SURXEY POINT-3 & & & 40 & 19 & & 5 & 49 & & 56 & 51 & & 5? & & & 21 & 22 & & 26 & 22 & & 26 & 23 & & 26 \\
\hline SUARY - I\$2 & & & 31 & $12 !$ & 59 & 5) & 107 & se & st & 110 & 56 & 55 & & & 14 & 55 & 26 & 24 & 49 & 25 & 25 & 50 & 26 & 25 \\
\hline $20055501-2$ & & & & & g & 46 & 45 & 46 & & 46 & 47 & 4 & & & & & 1 & 20 & 19 & 20 & & 20 & 20 & 20 \\
\hline BRUNSVICK-2 & & & & & 59 & & 156 & 159 & & 159 & 18 & & & & & & 11 & & 29 & 30 & & 10 & 30 & 0 \\
\hline LA SALLE - 1 HZ & & & & & 78 & $4 ! 2$ & & 391 & 399 & & $40]$ & 406 & & & & & 14 & 75 & & 71 & 72 & & 74 & $n$ \\
\hline PRAIRIE TSLANG-I R & & & & & 21 & 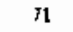 & 69 & $n$ & 72 & 12 & 12 & 73 & & & & & 8 & 28 & 28 & 28 & 29 & 29 & 29 & 29 \\
\hline PEACH BOTTONA-Z & & & & & 67 & 221 & & 222 & 223 & & 228 & 231 & & & & & 12 & 40 & & 40 & 41 & & 42 & 42 \\
\hline PEACH BOTTQM-3 & & & & & 58 & 223 & & 223 & 225 & & 229 & 232 & & & & & 11 & 41 & & 41 & $\$ 1$ & & $\$ 2$ & 42 \\
\hline BRUMSWICK-I & & & & & & 59 & 156 & & 150 & 160 & & 162 & & & & & & li & 29 & & 10 & xo & & 30 \\
\hline LA CAOSX: & & & & & & 11 & 21 & 22 & 22 & 22 & 22 & 22 & & & & & & 1 & 2 & 2 & 2 & 2 & 2 & 2 \\
\hline GIMNA & & & & & & 25 & 25 & 25 & 25 & 25 & 26 & $\mathbf{x}$ & & & & & & 9 & 9 & 9 & 9 & 9 & 9 & 4 \\
\hline FITZPATRICI & & & & & & & 168 & & 176 & $m$ & & 180 & & & & & & & 31 & & 32 & 32 & & 33 \\
\hline LALYERT GLIFF5-1 \&Z & & & & & & & & 1 & 130 & 65 & 65 & 331 & & & & & & & & & 51 & 25 & 25 & 51 \\
\hline PILGRIM-1 & & & & & & & & 92 & & 111 & 172 & & & & & & & & & 16 & & 30 & 30 & o \\
\hline INDTAM PUINT-2 & & & & & & & & & 18 & & 65 & 66 & & & & & & & & & 8 & & $x$ & 30 \\
\hline OHSTER CREEK & & & & & & & & & $19 t$ & & 17 & 192 & & & & & & & & & 35 & & 31 & $3 z$ \\
\hline COOPER & & & & & & & & & 57 & 102 & 102 & 102 & & & & & & & & & 10 & 19 & IB & 19 \\
\hline SEOUOYAH-LBZ & & & & & & & & & B & 75 & 75 & 152 & & & & & & & & & 4 & 33 & 33 & 68 \\
\hline ARKAHSAS MYCL DWE-1 & & & & & & & & & & 1 & 61 & & & & & & & & & & & & 28 & 0 \\
\hline gYROX-1 $<2$ & & & & & & & & & & $x 8$ & 102 & 100 & & & & & & & & & & 16 & $4]$ & 44 \\
\hline [HOIAH POINT-] & & & & & & & & & & 36 & & 69 & & & & & & & & & & 16 & & 32 \\
\hline DAYI5-BESSE- $!$ & & & & & & & & & & 38 & 45 & & & & & & & & & & & 18 & 21 & 0 \\
\hline POINT GEACH-LDZ & & & & & & & & & & 29 & 62 & 62 & & & & & & & & & & 7 & $2 z$ & 22 \\
\hline $21 O N-112$ & & & & & & & & & & & $\infty$ & $\$$ & & & & & & & & & & & 18 & 40 \\
\hline BEAVER YALLEY-1 & & & & & & & & & & & 47 & & & & & & & & & & & & 22 & 0 \\
\hline FORT CMHOUN-1 & & & & & & & & & & & 18 & $\omega 0$ & & . & & & & & & & & & 6 & 11 \\
\hline WHINE YAMKEE & & & & & & & & & & & & 34 & & & & & & & & & & & & g \\
\hline YERMONT YANKSE & & & & & & & & & & & & 36 & & & & & & & & & & & & 7 \\
\hline$N \mid N P-2$ & & & & & & & & & & & & 27 & & & & & & & & & & & & 5 \\
\hline PHA TOTAL & 50 & to & 259 & 298 & 403 & 483 & 553 & 501 & 664 & 837 & 1156 & 1372 & 20 & 28 & 11 & 128 & 169 & 200 & 235 & 211 & 281 & 356 & 490 & 585 \\
\hline BKR TOTAL & & 49 & & 172 & 262 & 1101 & 592 & 2284 & 1725 & 884 & 1769 & 1665 & & 9 & & 30 & 48 & 190 & 107 & 232 & 310 & 160 & 319 & 301 \\
\hline $\operatorname{cotal}$ & 50 & 119 & 259 & 467 & 665 & 1584 & 1145 & 1785 & 2309 & I721 & 2925 & 3037 & 20 & 37 & 11 & 159 & 217 & ans & $34]$ & 443 & 591 & 516 & 809 & ค月० 6 \\
\hline
\end{tabular}




\section{TABLE A.6. Middle Case Maximum At-Reactor Capacity - Projected Cumulative Storage Requirements}

\begin{tabular}{|c|c|c|c|c|c|c|c|c|c|c|c|c|c|c|c|c|c|c|c|c|c|c|c|c|c|}
\hline \multirow[b]{2}{*}{ Poest } & \multicolumn{12}{|c|}{ S5SEMBLLES } & \multicolumn{13}{|c|}{$M+$ ในM } \\
\hline & 1466 & 1997 & 1968 & 14요 & 1990 & $199 !$ & 1992 & 1993 & 1994 & 1895 & 1996 & 1997 & 1986 & 1987 & 198 & & $949 \quad 1$ & 1990 & 19911 & $1992 \quad 19$ & $993 \quad 19$ & $1994 \quad 17$ & $1795 \quad 19$ & $1995 \quad 1$ & 1997 \\
\hline HILLSTOHE-? & 50 & $10 y$ & 178 & $1 / 8$ & 248 & 314 & 379 & 319 & 444 & 509 & $5 / 5$ & 575 & 29 & 44 & & 72 & 12 & 100 & $2: 27$ & 153 & 153 & 180 & 206 & $\overline{232}$ & $23 ?$ \\
\hline$\therefore A L I S A D E S$ & & 2 & SN & 50 & 120 & 179 & Itg & 259 & 299 & 299 & 360 & a22 & & 1 & & 23 & 23 & 13 & 69 & 50 & 93 1 & $116 \quad l$ & 116 J & 340 & 104 \\
\hline :URXEY PUINT-A & & 5 & 51 & 61 & 116 & 173 & 173 & 228 & 298 & 286 & 342 & 402 & & 3 & & 28 & 29 & 53 & 19 & $79 \quad 1$ & 105 & 131 & 131 & 157 & 1855 \\
\hline 5T. LULUE-I & & 3 & 3 & 15 & 145 & 145 & 218 & 290 & 289 & 363 & 435 & 435 & & I & & 1 & 27 & 53 & 53 & 801 & $105 \quad 10$ & 105 & 1321 & 159 & 159 \\
\hline 14:LLรTUME-1 & & $4 y$ & 49 & 221 & 221 & 394 & 394 & 570 & 145 & 745 & 925 & 925 & & g & & 9 & 39 & 39 & 30 & 70 & $101 \quad 1$ & 131 & 131 & 163 & 163 \\
\hline TLRKEYY DOLNT-3 & & & 46 & 95 & 95 & $15 i$ & 200 & 200 & 25 & $30 t$ & 307 & 364 & & & & 21 & 44 & 44 & 69 & 92 & 92 & 118 & $16 \mathrm{I}$ & 141 & 167 \\
\hline SURAX -102 & & & ओ & 152 & 210 & 263 & 370 & $\$ 24$ & 478 & 589 & 541 & 699 & & & & 14 & 59 & 96 & 120 & $169 \quad 1$ & 1932 & 219 & 256 & 293 & 318 \\
\hline BCONE E - 3 & & & & 53 & 113 & 113 & 173 & 173 & 234 & 295 & 295 & 357 & & & & & 75 & 52 & 52 & 90 & 80 & 109 & 137 & 13 & $16 k$ \\
\hline AOH ] MSOH-Z & & & & & 7 & 55 & 100 & 146 & 106 & 192 & 239 & 298 & & & & & & 4 & 24 & 43 & 63 & 63 & 33 & 203 & 123 \\
\hline SALUNSWICK-2 & & & & & 59 & 59 & 215 & 373 & 373 & 532 & 694 & 694 & & & & & & 11 & 11 & 40 & n & 70 & 99 & 100 & 130 \\
\hline LA SALLE-1\$? & & & & & 78 & 490 & 490 & B日 1 & 1280 & 1280 & {$[68]$} & 2089 & & & & & & 14 & 99 & 89 & 165 & 232 & 237 & $31)$ & $38 n$ \\
\hline PHALRIE ISLANDL-LA? & & & & & 21 & 92 & 161 & 232 & 304 & 3/6 & 448 & 521 & & & & & & 8 & 37 & 54 & 93 & 122 & 150 & 179 & 209 \\
\hline DEACH BOTROH-2 & & & & & 67 & 268 & $28 \mathrm{e}$ & 510 & 733 & 233 & 961 & 1192 & & & & & & 12 & $\$ 2$ & 52 & 93 & 134 & 13a & 175 & टा \\
\hline$P \notin A C H$ BO rTUth-3 & & & & & 59 & 281 & 281 & $5 n d$ & 129 & 120 & $95 \mathrm{H}$ & $119 n$ & & & & & & 11 & si & \&1 & $9 ?$ & 193 & 133 & 174 & 217 \\
\hline BKUNSW][k-1 & & & & & & 59 & 215 & 215 & m3 & 533 & 533 & 695 & & & & & & & 11 & $4 \pi$ & an & $m$ & inn & $\ln$ & 130 \\
\hline LA CKLISSE & & & & & & [1] & 32 & $\mathbf{s}$ & 16 & 98 & 120 & 142 & & & & & & & 1 & 3 & 6 & 9 & 11 & 13 & 15 \\
\hline WCUAEE-IAZ & & & & & & 50 & 110 & 228 & 285 & 305 & 465 & 524 & & & & & & & 23 & 51 & ID6 & 132 & 160 & 216 & 243 \\
\hline INNTILELLO & & & & & & 2 & 93 & 93 & 196 & 279 & 373 & 458 & & & & & & & & 19 & 16 & 33 & 49 & $5 \pi$ & BI \\
\hline |, ANIA & & & & & & 25 & 50 & 75 & 100 & 125 & 151 & 177 & & & & & & & 9 & 18 & 26 & 35 & $d t$ & 53 & 62 \\
\hline =ITLPATH [GK & & & & & & & 16 & 169 & 344 & 521 & 521 & to1 & & & & & & & & $\$ 1$ & э & 53 & 35 & 95 & 128 \\
\hline CALVERT CLIFFS-:d2 & & & & & & & & 1 & [3! & 196 & 261 & 392 & & & & & & & & & & 51 & if & !0? & 153 \\
\hline ILGRS SM=1 & & & & & & & & 42 & 92 & 263 & 435 & 435 & & & & & & & & & 16 & 16 & 4) & $n$ & $n$ \\
\hline INULAN PUIHI-Z & & & & & & & & & 18 & 18 & 83 & 199 & & & & & & & & & & 8 & 马 & 28 & 58 \\
\hline ISYSIER CHEEK & & & & & & & & & 197 & 197 & 374 & 556 & & & & & & & & & & 35 & 35 & 56 & 98 \\
\hline एथOPER & & & & & & & & & s? & 159 & $26 !$ & 363 & & & & & & & & & & 10 & 29 & 48 & 56 \\
\hline SELYUUYAH - 1 A2 & & & & & & & & & ค & 93 & 158 & 310 & & & & & & & & & & 4 & 37 & $3 \pi$ & 138 \\
\hline LRKANSAS RUCL UNE -: & & & & & & & & & & 1 & 52 & 62 & & & $\cdot$ & & & & & & & & & 29 & 2 \\
\hline AYRUM-]AZ & & & & & & & & & & 19 & 140 & 244 & & & & & & & & & & & 45 & 59 & 103 \\
\hline |MUIAN POCRTT-3 & & & & & & & & & & 36 & 36 & 105 & & & & & & & & & & & 16 & ifi & 18 \\
\hline OAV [S- DE SSE-I & & & & & & & & & & 28 & 93 & 93 & & & & & & & & & & & 19 & 39 & 39 \\
\hline SIINT AEACH-L & & & & & & & & & & 20 & 82 & 144 & & & & & & & & & & & r & 29 & 51 \\
\hline $21 U N-1+2$ & & & & & & & & & & & 40 & $12 \mathrm{~B}$ & & & & & & & & & & & & 18 & 59 \\
\hline SE.AVI \& YALLEEY & & & & & & & & & & & 4) & 47 & & & & & & & & & & & & $\eta$ & $2 ?$ \\
\hline FIORT GALHTHUT-I & & & & & & & & & & & 19 & $S B$ & & & & & & & & & & & & 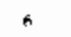 & 21 \\
\hline SKACUHOUL-1S2 & & & & & & & & & & & & 64 & & & & & & & & & & & & & 27 \\
\hline$\therefore c(1) 3 k-1 d 2$ & & & & & & & & & & & & $n$ & & & & & & & & & & & & & 31 \\
\hline 'HA:HE YATUKEE & & & & & & & & & & & & 24 & & & & & & & & & & & & & 9 \\
\hline VERIIUNT TMRKFE & & & & & & & & & & & & 36 & & & & & & & & & & & & & $r$ \\
\hline wrtp-2 & & & & & & & & & & & & $\eta$ & & & & & & & & & & & & & s \\
\hline PUR TOTL & so & 120 & 379 & 674 & $10 n$ & 1560 & 2113 & 2614 & 3278 & 4115 & 5271 & & & 20 & $\$ 9$ & 160 & $28 \mathrm{~g}$ & 457 & 663 & 908 & 1110 & 139 & Ifd & 2237 & 2822 \\
\hline BพA тоти & & 49 & 49 & 221 & 483 & 1584 & 2176 & 3460 & 5185 & 6069 & 1830 & 9503 & & & $g$ & 9 & 39 & 97 & 286 & 393 & 625 & 935 & 1094 & 1413 & 1314 \\
\hline TOTAL & 50 & 169 & 428 & 995 & 1560 & 3144 & 4289 & 6074 & 8483 & 10194 & 13109 & 16146 & & 20 & 57 & 168 & 327 & 544 & 949 & 1292 & 1334 & 2326 & 2841 & 3650 & 4536 \\
\hline
\end{tabular}




\section{TABLE A.7. Middle Case Maxinfum At-Reactor Capacity - P1us Transshipment - Projected Annual Storage Requirements}

ASSEMoltes

\begin{tabular}{|c|c|c|c|c|c|c|c|c|c|c|c|c|c|c|c|c|c|c|c|c|c|c|c|}
\hline \multirow[b]{2}{*}{ PNOL } & & \\
\hline & $\underline{1986}$ & $\underline{19 \theta 7} \underline{1988}$ & $\underline{1999}$ & $\underline{1990}$ & $\underline{691}$ & 1992 & $\underline{1993}$ & 1994 & $\underline{1995}$ & $\underline{1996}$ & $\underline{1997}$ & 1996 & $\underline{1987}$ & $\underline{1980}$ & 1989 & $\underline{199 \pi}$ & 1991 & $\underline{1992}$ & $\underline{1993}$ & $\underline{1992}$ & $\underline{1995}$ & $\underline{1996}$ & 1497 \\
\hline MILLSTONE-T & & 49 & 172 & & $1 / 3$ & & 176 & 175 & & 180 & & & 9 & & ]0 & & 31 & & $3\}$ & 31 & & 32 & 7 \\
\hline PRA]R[E [SLAMD-SAZ & & & & 21 & 71 & $\theta$ & 71 & 12 & 2 & 12 & 73 & & & & & B & $2 B$ & 29 & 78 & $\not x$ & 79 & 20 & 29 \\
\hline BRUKSWLCK-1 & & & & & 119 & 156 & & 158 & 16n & & 162 & & & & & & 22 & 29 & & in & 30 & & $3 n$ \\
\hline LA CROSSE & & & & & 11 & 21 & 22 & 22 & 22 & 72 & 22 & & & & & & 1 & 2 & $?$ & 2 & 2 & 2 & 2 \\
\hline ONTICELLO & & & & & $z$ & 91 & & 93 & 93 & 98 & AS & & & & & & & 16 & & $: 6$ & 16 & 1) & 15 \\
\hline GIMMA & & & & & 25 & 25 & 25 & 25 & 25 & $2 \pi$ & 26 & & & & & & 9 & 9 & 9 & 9 & 9 & 9 & 9 \\
\hline ST. LUC:E-1 & & & & & & 27 & 71 & & 74 & 12 & & & & & & & & 3 & 26 & & 23 & 26 & $n$ \\
\hline TURKEY DULMT-3 & & & & & & & & 56 & 51 & & 57 & & & & & & & & & 26 & 23 & & 26 \\
\hline TURKEY $P O C N T=4$ & & & & & & & 59 & sin & & 56 & 50 & & & & & & & & 25 & 27 & & 25 & $2 \theta$ \\
\hline ST. LUCIE-2 & & & & & & 68 & $n$ & & 5) & 19 & & & & & & & & 26 & 29 & & 25 & 30 & 0 \\
\hline FITLPATAICK & & & & & & $i \in$ & & 176 & 377 & & 180 & & & & & & & $3 t$ & & 32 & 32 & & 33 \\
\hline EALYERT CLLFF5.152 & & & & & & & 1 & 130 & 65 & 65 & 131 & & & & & & & & & 51 & 25 & 25 & 51 \\
\hline PLLGRIM-1 & & & & & & & 92 & & 111 & 172 & & & & & & & & & t6 & & 30 & 30 & T \\
\hline [HOLAN PD]NT-? & & & & & & & & 18 & & 85 & 66 & & & & & & & & & a & & $3 n$ & 30 \\
\hline UYSTER CAEEKK & & & & & & & & 191 & & $|7\rangle$ & 192 & & & & & & & & & 35 & & 31 & 32 \\
\hline MORTH AMMA-18Z & & & & & & & & $\$ 1$ & 61 & 124 & 63 & & & & & & & & & 28 & 28 & 5) & 79 \\
\hline [MDLAM PD)]NT-3] & & & & & & & & & $3 \sqrt{4}$ & & 69 & & & & & & & & & & 16 & & 32 \\
\hline DAVIS-BESSE- 1 & & & & & & & & & 개 & a5 & & & & & & & & & & & 18 & 21 & n \\
\hline POLNT BEACH- L\$2 & & & & & & & & & $3 n$ & 52 & $A 2$ & & & & & & & & & & 7 & 22 & 22 \\
\hline BXRLN- 152 & & & & & & & & & & 37 & 104 & & & & & & & & & & & 16 & Ad \\
\hline $210 M-182$ & & & & & & & & & & & $A B$ & & & & & & & & & & & & 40 \\
\hline 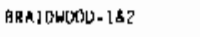 & & & & & & & & & & In & 104 & & & & & & & & & & & d4 & 44 \\
\hline FJRT CALHOUUN-] & & & & & & & & & & 18 & 40 & & & & & & & & & & & k & 14 \\
\hline PALISADES & & & & & & & & & & & 52 & & & & & & & & & & & & 24 \\
\hline MLOLANO-IAR & & & & & & & & & & & 57 & & & & & & & & & & & & 27 \\
\hline $0<\cos k-1 d 2$ & & & & & & & & & & & 11 & & & & & & & & & & & & $3 t$ \\
\hline HALIME YANKEE & & & & & & & & & & & 24 & & & & & & & & & & & & 9 \\
\hline PEACH BOITCM-3 & & & & & & & & & & & 35 & & & & & & : & & & & & & 6 \\
\hline$L$ [MERICK - I & & & & & & & & & & & 224 & & & & & & & & & & & & $\$ 1$ \\
\hline LIMERICK-Z & & & & & & & & & & & 213 & & & & & & & & & & & & 39 \\
\hline YERMONT XAMKEE & & & & & & & & & & & 36 & & & & & & & & & & & & 7 \\
\hline PWR TQTAL & & & & 21 & 96 & 184 & $\operatorname{moc}$ & 451 & $6: 9$ & ค90 & 1212 & & & & & $\mathrm{~g}$ & 37 & 70 & IIA & 191 & 25A & उбБ & 513 \\
\hline RUL TRTAL & & 19 & $m$ & & 304 & 592 & A4g & 878 & 9: & an9 & $125 \mathrm{~A}$ & & a & & in & & 54 & In? & 19 & 156 & 100 & 1ร1 & $? 9$ \\
\hline rarel & & 99 & 172 & 21 & $\operatorname{ano}$ & 736 & 748 & 1329 & דחר & $1 / 80$ & 7420 & & 9 & & in & A & 91 & 17 & 197 & 347 & 417 & 537 & $78 ?$ \\
\hline
\end{tabular}




\section{TABLE A.8. Middie Case Maximum At-Reactor Capacity - Plus Transshipment - Projected Cumulative Storage Requirements}

\begin{tabular}{|c|c|c|c|c|c|c|c|c|c|c|c|c|c|c|c|c|c|c|c|c|c|c|c|c|}
\hline \multirow[b]{2}{*}{ POOL } & \multicolumn{12}{|c|}{ ASSEMBLL LES } & \multicolumn{12}{|c|}{ tilum } \\
\hline & 1986 & 1987 & 1988 & 1989 & 1990 & 1991 & $\underline{1992}$ & 1993 & 199 & $\underline{1995}$ & 1996 & 1999 & 19860 & $\underline{1987}$ & 1989 & 19899 & 1990 & 1991 & 1992 & $\underline{1993}$ & 1994 & 1995 & 1996 & $\underline{1997}$ \\
\hline MLLLSTOME-1 & & 49 & 49 & 221 & 221 & 394 & 594 & 570 & 745 & 745 & 925 & 925 & & 8 & 9 & 39 & 9 & 70 & "n & 101 & 131 & 131 & 163 & 163 \\
\hline PRA1R LE ISLAMD-152 & & & & & 21 & 92 & 161 & 232 & 304 & 376 & $4 \angle 8$ & 521 & & & & & B & 37 & sis & 93 & 122 & 150 & 179 & 208 \\
\hline BRUMSHCLX -1 & & & & & & 118 & 274 & 274 & $\$ 32$ & $\$ 92$ & 592 & 354 & & & & & & 22 & 51 & 51 & B1 & 111 & III & 14] \\
\hline ENUNSNGCK-Z & & & & & & & 156 & 314 & 314 & 473 & 635 & 635 & & & & & & & 29 & 59 & S & $8 B$ & 199 & 119 \\
\hline LA CRUSSE & & & & & & at & 32 & $s$ & 76 & 98 & 120 & 142 & & & & & & 1 & 3 & 6 & 8 & 11 & 13 & 15 \\
\hline WNTJCELLO & & & & & & 2 & 93 & 93 & 286 & 279 & $3 / 3$ & 459 & & & & & & & 16 & 16 & 33 & 49 & 66 & a1 \\
\hline Ginue & & & & & & 25 & 50 & 75 & $1 \infty 0$ & 125 & 151 & 177 & & & & & & 9 & 18 & 26 & 35 & 4 & 53 & 52 \\
\hline ST. LUCTE-I & & & & & & & 22 & 93 & 93 & 167 & 239 & 239 & & & & & & & 8 & 3 & 34 & 61 & 87 & 97 \\
\hline TURKEY POLNT-3 & & & & & & & & & 56 & 707 & 107 & 164 & & & & & & & & & 26 & 49 & 49 & 35 \\
\hline TUнKEY PO:RT-4 & & & & & & & & 55 & 113 & 113 & 169 & 289 & & & & & & & & 25 & 52 & 52 & a & 105 \\
\hline 5T. LUCIE-? & & & & & & & $\$ 8$ & !45 & 105 & 212 & 291 & 291 & & & & & & & 26 & 55 & 55 & ค1 & 121 & 111 \\
\hline SITZPATRICK & & & & & & & $1 \notin 8$ & 168 & 34 & 521 & 521 & 101 & & & & & & & 1 & 31 & 63 & 95 & 95 & 129 \\
\hline CALVERT CLIFSS-1S: & & & & & & & & 1 & 131 & 196 & 261 & 392 & & & & & & & & & 51 & 76 & 102 & 153 \\
\hline DLGRIM-1 & & & & & & & & 97 & 92 & 253 & 435 & 435 & & & & & & & & 16 & 16 & 47 & $n$ & I7 \\
\hline :NOLAN DOSHT -2 & & & & & & & & & 18 & 18 & $\mathrm{B3}$ & 149 & & & & & & & & & 9 & 8 & X8 & SA \\
\hline OYSTER CHEEK & & & & & & & & & 191 & 197 & 374 & 556 & & & & & & & & & 35 & 35 & 66 & 98 \\
\hline COUPER & & & & & & & & & 57 & 159 & 261 & 363 & & & & & & & & & 10 & 29 & sa & 66 \\
\hline $5 \operatorname{LSRR} Y-1 \$ z$ & & & & & & & & & 31 & 141 & 197 & 252 & & & & & & & & & 14 & 64 & 89 & 115 \\
\hline HURTH ANNA-162 & & & & & & & & & 51 & 122 & 246 & 309 & & & & & & & & & 28 & 56 & 113 & 142 \\
\hline [MUCAN PO]NT-] & & & & & & & & & & 36 & 36 & 105 & & & & & & & & & & 16 & 16 & 48 \\
\hline JAYLS- UESSE-1 & & & & & & & & & & $\mathbf{p}$ & 93 & 83 & & & & & & & & & & 18 & 59 & 39 \\
\hline POINT BEACH- 152 & & & & & & & & & & 20 & $B 2$ & 244 & & & & & & & & & & 1 & 29 & 51 \\
\hline BYRUM-1\$2 & & & & & & & & & & & 37 & 141 & & & & & & & & & & & 16 & 60 \\
\hline $210 \mathrm{~N}-1+2$ & & & & & & & & & & & & 86 & & & & & & & & & & & & 40 \\
\hline BRATDWOOD-1S2 & & & & & & & & & & & 103 & 207 & & & & & & & & & & & 44 & 88 \\
\hline FORT CALHOUN-I & & & & & & & & & & & 18 & 58 & & & & & & & & & & & 6 & 21 \\
\hline PALISADES & & & & & & & & & & & & 52 & & & & & & & & & & & & 24 \\
\hline MIOLANO-IdZ & & & & & & & & & & & & 57 & & & & & & & & & & & & 27 \\
\hline $0 \in \cos -1 s 2$ & & & & & & & & & & & & $n$ & & & & & & & & & & & & 31 \\
\hline MAINE YANGEE & & & & & & & & & & & & 24 & & & & & & & & & & & & 9 \\
\hline PEACH BOTIDH-3 & & & & & & & & & & & & 35 & & & & & & & & & & & & $\delta$ \\
\hline LIMERICK-I & & & & & & & & & & & & 274 & & & & & & & & & & & & Al \\
\hline LIMERILK-Z & & & & & & & & & & & & 213 & & & & & & & & & & & & 39 \\
\hline VERHUNY YAMEEE & & & & & & & & & & & & 36 & & & & & & & & & & & & 7 \\
\hline WNP - - & & & & & & & & & & & & 27 & & & & & & & & & & & & 5 \\
\hline Phit IIJTAL & & & & & 21 & 111 & 301 & 501 & 1052 & 1671 & 2551 & 3763 & & & & & 8 & 46 & 116 & 234 & A25 & SA2 & 1049 & $15 \delta 2$ \\
\hline IR TDTAL & & 99 & 49 & 221 & 221 & 525 & 1117 & 1565 & 2043 & 2321 & 4236 & 5504 & & 9 & 9 & 30 & 39 & 93 & 200 & 280 & 436 & $\$ 96$ & 757 & YHG \\
\hline Tof $\mathrm{Ac}$ & & 49 & 44 & 221 & 242 & 642 & $141 \mathrm{~B}$ & 166 & 3495 & $199 R$ & 5787 & 9267 & & 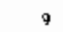 & 9 & 39 & A? & 139 & 316 & ૬६४ & 61 & 1279 & AnIS6 & 254 \\
\hline
\end{tabular}


APPENDIX B

MAP OF FEDERAL. ENERGY REGIONS 


\section{MAP OF FEDERAL. ENERGY REGIONS}

The following map of the federal regions (Figure 3.1) shows the geographical location of the generic reactor sites. Each generic reactor is located at a designated reactor site within the region. The reactor site selected is located as nearly as possible to the centroid of the region. It is not predicted that all of the generic reactors will be located at the within-region site. This method of allocation merely assures some site diversity for logistics study purposes. 


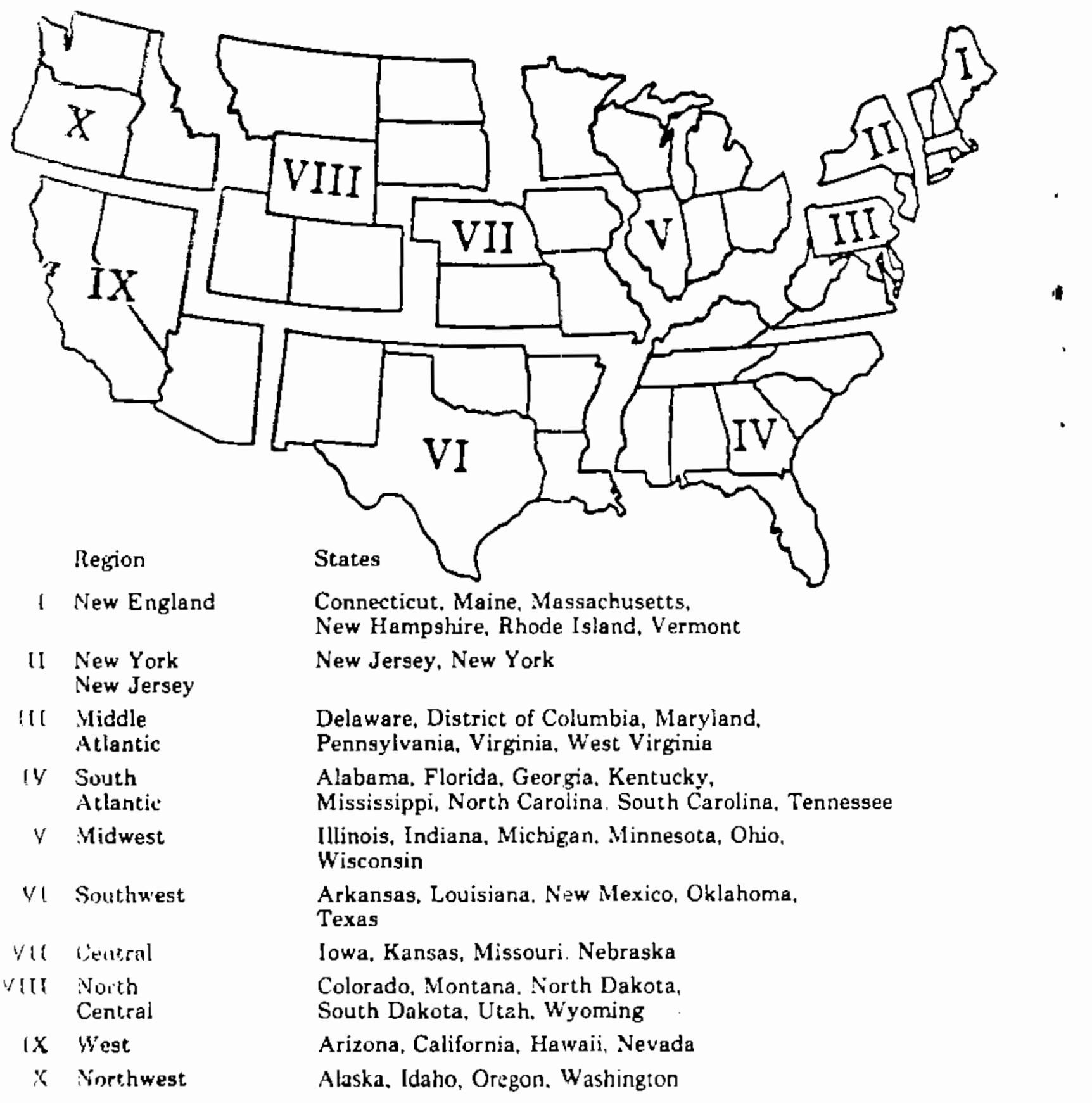

FIGURE B.1. Federa? Energy Fiegions 


\section{DISTRIBUTION}

No. of

Copies

OFFSITE

R. H. Bauer

$R H-10$

Office of Civilian Radioactive Maste Management

U.S. Department of Energy

Washington, D.C. 20585

R. W. Bown

$R W-30$

Office of Civilian Radioactive Waste Management

U.S. Department of Energy

Washington, D.C. 20585

J. H. Carison

$\mathrm{RH}-32$

Office of Civilian Radioactive Waste lanagement

U.S. Department of Energy

Washington, D.C. 20585

R. G. Clark

Director of Nuclear and A) ternate Fuels (EI-53)

U.S. Department of Energy

Washington, D.C. 20585

C. W. Conner

$\mathrm{RW}-33$

Office of Civilian Radioactive Waste Management

U.S. Department of Energy

Washington, D.C. 20585

J. Disbrow

EI-53I

Energy Information Administration

U.S. Department of Energy Washington, D.C. 20585
No. of

Copies

\section{J. Epstein}

$\mathrm{RW}-32$

Office of Civilian Radioactive Waste Management

U.S. Department of Energy

Washington, D.C. 20585

F. P. Falci

$D P-123$

U.S. Department of Energy

Washington, D.C. 20545

J. S. Finucane

EI-53I

Energy Information Administration

U.S. Department of Energy

Washington, D.C. 20585

W. A. Frankhauser

DP -122.1

U.S. Department of Energy

Washington, D.C. 20545

J. R. Hilley

$\mathrm{RW}-30$

Office of Civilian Radioactive Waste Management

U.S. Department of Energy

Washington, D.C. 20585

T. H. Is aacs

$\mathrm{RW}-42$

Office of Civilian Radioactive Waste Management

U.S. Department of Energy

Washington, D.C. 20585

K. A. Klein

$\mathrm{RW}-32$

Office of Civilian Radioactive Waste Management

U.S. Department of Energy

Washington, D.C. 20585 
No. of

Copies

E. F. Mastel

$\mathrm{NE}-72$

U.S. Department of Energy

Washington, D.C. 20545

20 R. A. Milner

$\mathrm{RW}-12$

U.S. Department of Energy

Washington, D.C. 20545

T. D. Nguyen

$\mathrm{R} W-33$

Office of Civilian Radioactive Waste Management

U.S. Department of Energy

Washington, D.C. 20585

M. L. Payton

$\mathrm{R} W-12$

Office of Civilian Radioactive Waste Management

U.S. Department of Energy

Washington, D.C. 20585

R. Philpott

$\mathrm{RW}-33$

Office of Civilian Radioactive Waste Management

U.S. Department of Energy

Washington, D.C. 20585

D. E. Shelor

$\mathrm{RW}-32$

Office of Civilian Radioactive Waste Management

U.S. Department of Energy

Washington, D.C. 20585

H. Stejnberg

EI-532

Energy Information

Administration

U.S. Department of Energy

Washington, D.C. 20585
No. of

Copies

\author{
W. M. Sprecher \\ $\mathrm{RW}-42$ \\ Office of Civilian Radioactive \\ Waste Management \\ U.S. Department of Energy \\ Washington, D.C. 20585 \\ E. L. Wilmont \\ $\mathrm{RW}-33$ \\ Office of Civilian Radioactive \\ Waste Management \\ U.S. Department of Energy \\ Washington, D.C. 20585 \\ R. C. Ashline \\ Oak Ridge National \\ Laboratory \\ P.0. Box $X$ \\ Oak Ridge, TN 37830 \\ C. W. Forsberg \\ Oak Ridge National \\ Laboratory \\ P.0. Box $X$ \\ Oak Ridge, TN 37830 \\ J. A. Klein \\ Oax Ridge National \\ Laboratory \\ P.0. Box $x$ \\ Oax Ridge, TN 37830 \\ D. S. Joy \\ Oak Ridge National \\ Laboratory \\ P.0. Box $X$ \\ Oak Ridge, TN 37830 \\ Ken Golleher \\ DOE Albuquerque Operations \\ office \\ P.0. Box 5400 \\ Albuquerque, NM 87115 \\ G. C. Allen \\ Sandia National Laboratories \\ P.0. Box 5800 \\ Albuquerque, NM 87185
}


No. of

Copies

J. W. Cashwell

Sandia National Laboratories

P.0. Box 5800

Alhuquerque, NM 87185

E. R. Johnson

JAI

11702 Bowman Green

Reston, VA 22090

R. W. Lambert

Electric Power Research institute

$34: 2$ Hillview Avenue

Pa`o Alto, CA 94303

R. F. Willians

Electric Power Research

Inst itute

3412 Hillview Avenue

Palo Alto, CA 94303

R. P. Morissette

G.A. Technologies

P.0. Box 85608

San Diego, CA 92138

B. F. Judson

General Electric Company

175 Cortner Avenue

San Jose, CA 95630

Yong Park

NUS Corporation

910 Clopper Road

Gaithersburg, MD 20878

B. R. Teer

Transnuciear, Inc.

One North Broadway

White Plains, NY 10601

Edward Kuhn

Stone \& Webster Engineering

Corp.

One Penn Plaza

250 West 34 th Street

New York, NY 10119
No. of

Copies

Ralph Best

7938 Sarahurst

Dublin, OH 43017

J. H. Saling

Westinghouse Electric Corp.

Waste Technology Service

Division

Headquarters Site

P.0. Box 286

Madison, PA 15663

3 Frank Haines

Roy $F$. Weston

2301 Research Blvd.,

Third Floor

Rockville, MD 20850

30 DOE Technical Information

Center

ONSITE

6 DOE Richland Operations office

P. A. Craig (2)

0. Crouter

D. C. Langstaff

M. J. Plahuta

H. E. Ranson

64 Pacific Northwest Laboratory

W. J. Bailey

W. W. Ballard

G. H. Beeman

J. L. Braitman

B. M. Cole

C. A. Counts

J. M. Creer

P. M. Daling

L. R. Dodd

A. L. Doherty

K. A. Ekblaw

R. L. Engel

J. F. Fletcher

E. R. Gilbert

R. J. Hall 
No. of

Copies

C. M. Heeb

J. N. Holloway

G. M. Holter

A. B. Johnson

G. J. Konzek

R. A. Libby (15)

W. W. Little

A. T. Luksic

J. L. McEl roy

R. H. McKee

G. H. McNair

E. T. Merrill

J. F. Nesbitt

D. F. Newman

D. R. Oden
No. of

Copies

D. R. Payson

W. L. Purcell

R. G. Rau

G. E. Russcher

K. J. Schneider

M. R. Shay

M. J. Sherbin

R. I. Smith

L. A. Strope

M. B. Triplett

R. C. Walling

M. K. White

T. W. Wood

Publishing Coordination $\mathrm{MH}$ (2)

Technical Information (5) 